\title{
The Sun and the Earth's Climate
}

\author{
Joanna D. Haigh \\ Imperial College \\ London SW7 2AZ UK \\ email: j.haigh@imperial.ac.uk \\ http://imperial.ac.uk/people/j.haigh \\ Living Reviews in Solar Physics \\ ISSN 1614-4961 \\ Accepted on 18 September 2007 \\ Published on 2 October 2007
}

\begin{abstract}
Variations in solar activity, at least as observed in numbers of sunspots, have been apparent since ancient times but to what extent solar variability may affect global climate has been far more controversial. The subject had been in and out of fashion for at least two centuries but the current need to distinguish between natural and anthropogenic causes of climate change has brought it again to the forefront of meteorological research. The absolute radiometers carried by satellites since the late 1970s have produced indisputable evidence that total solar irradiance varies systematically over the 11-year sunspot cycle, relegating to history the term "solar constant", but it is difficult to explain how the apparent response to the Sun, seen in many climate records, can be brought about by these rather small changes in radiation. This article reviews some of the evidence for a solar influence on the lower atmosphere and discusses some of the mechanisms whereby the Sun may produce more significant impacts than might be surmised from a consideration only of variations in total solar irradiance.
\end{abstract}

This review is licensed under a Creative Commons Attribution-Non-Commercial-NoDerivs 2.0 Germany License. http://creativecommons.org/licenses/by-nc-nd/2.0/de/ 


\section{Imprint / Terms of Use}

Living Reviews in Solar Physics are published by the Max Planck Institute for Solar System Research, Max-Planck-Str. 2, 37191 Katlenburg-Lindau, Germany. ISSN 1614-4961

This review is licensed under a Creative Commons Attribution-Non-Commercial-NoDerivs 2.0

Germany License: http://creativecommons.org/licenses/by-nc-nd/2.0/de/

Because a Living Reviews article can evolve over time, we recommend to cite the article as follows:

Joanna D. Haigh,

"The Sun and the Earth's Climate",

Living Rev. Solar Phys., 4, (2007), 2. [Online Article]: cited [<date $>$ ], http://www.livingreviews.org/lrsp-2007-2

The date given as $<$ date $>$ then uniquely identifies the version of the article you are referring to.

\section{Article Revisions}

Living Reviews supports two different ways to keep its articles up-to-date:

Fast-track revision A fast-track revision provides the author with the opportunity to add short notices of current research results, trends and developments, or important publications to the article. A fast-track revision is refereed by the responsible subject editor. If an article has undergone a fast-track revision, a summary of changes will be listed here.

Major update A major update will include substantial changes and additions and is subject to full external refereeing. It is published with a new publication number.

For detailed documentation of an article's evolution, please refer always to the history document of the article's online version at http://www.livingreviews.org/lrsp-2007-2. 


\section{Contents}

1 Introduction $\quad 5$

2 Climate Records $\quad 6$

2.1 Measurements and reconstructions . . . . . . . . . . . . . . . . . . . . 6 6

2.2 Solar signals in surface climate . . . . . . . . . . . . . . . . . . . . . . . . . . . . . . . . . . .

2.3 Solar signals throughout the atmosphere . . . . . . . . . . . . . . . 13

3 Earth Radiation Budget and Radiative Forcing of Climate Change 20

3.1 Earth radiation budget . . . . . . . . . . . . . . . . . . 20

3.2 Radiative forcing of climate change . . . . . . . . . . . . . . . . . 20

4 Total Solar Irradiance $\quad 24$

4.1 The Earth's orbit around the Sun . . . . . . . . . . . . . . . . . . . . . 24

4.2 Variations in total solar irradiance . . . . . . . . . . . . . . . . . . 25

5 Solar spectral irradiance $\quad \mathbf{2 7}$

5.1 Absorption of solar spectral radiation by the atmosphere . . . . . . . . . . . . 27

5.2 Variability of solar spectral irradiance and heating rates . . . . . . . . . . . . . . . . 28

5.3 Stratospheric photochemistry . . . . . . . . . . . . . . . . . . . . . . . . . . . . . . . .

5.4 Solar cycle signal in stratospheric ozone . . . . . . . . . . . . . . . . . . . . 33

5.5 Impact of the stratosphere on solar radiative forcing of climate . . . . . . . . . 36

6 Models and Mechanisms $\quad 40$

6.1 Climate change in response to variations in total solar irradiance . . . . . . . . . 40

6.2 Model studies of the influence of varying UV in the middle atmosphere . . . . . . . 43

6.3 Dynamical mechanisms in the middle atmosphere . . . . . . . . . . . . . 45

6.4 Model studies of the influence of varying solar UV on the troposphere . . . . . . . 48

6.5 Coupling between the stratosphere and troposphere . . . . . . . . . . . . . 50

7 Clouds $\quad \mathbf{5 3}$

7.1 Clouds and the Earth radiative budget . . . . . . . . . . . . . . . . . . 53

7.2 Cloud formation . . . . . . . . . . . . . . . . . . . . . . 53

7.3 Atmospheric ionisation and cloud . . . . . . . . . . . . . . . . 54

8 Conclusions $\quad 58$

$\begin{array}{lr}\text { References } & 59\end{array}$ 



\section{Introduction}

At periods of higher solar activity the Earth is subject to enhanced solar irradiance, a greater incidence of solar energetic particles and fewer galactic cosmic rays. This review presents an assessment of the extent to which solar variability affects the climate of the Earth's lower and middle atmosphere and considers how the apparent response is brought about.

A summary of the potential routes whereby solar variability might influence the climate of the lower atmosphere is presented in Table 1 . The absorption of solar radiation determines the Earth's mean temperature and radiation budget, while the latitudinal distribution of the absorbed radiation is the primary driver for atmospheric circulations. Through photochemical processes solar radiation and solar energetic particles also play an important part in determining middle atmospheric composition. Now the radiative heating rate at any point in the atmosphere is the net effect of solar heating and infrared cooling, the latter being intrinsically related to the atmospheric composition and temperature structure. Thus variations in solar output have the potential to affect the atmospheric temperature structure in a complex and non-linear fashion. Section 3 below discusses the energy balance of the Earth and assesses the potential role of the Sun in the radiative forcing of global climate change. Section 4 considers how variations in the total solar irradiance incident at Earth may affect the climate while in Section 5 the processes involving solar ultraviolet radiation, and how these are modulated by solar activity, are presented. Galactic cosmic rays are an important cause of atmospheric ionisation and, as their intensity is modulated by solar activity, there is also a solar signal in the concentration of atmospheric ions which are involved in chemical processes in the middle atmosphere. To what extent they might also provide a source of cloud condensation nuclei at lower altitudes is an area of current research and this is the subject of Section 7.

Table 1: Summary of routes through which solar variability may influence the climate of the lower atmosphere.

\begin{tabular}{|l|l|}
\hline Forcing factor & Generic mechanism \\
\hline $\begin{array}{l}\text { Total solar irradiance (variations due } \\
\text { to orbital variations or to variable so- } \\
\text { lar emission). }\end{array}$ & $\begin{array}{l}\text { Radiative forcing of climate. Direct impact on sea } \\
\text { surface temperatures and hydrological cycle. }\end{array}$ \\
\hline Solar UV irradiance. & $\begin{array}{l}\text { Heating the upper and middle atmosphere, dy- } \\
\text { namical coupling down to troposphere. Middle and } \\
\text { lower atmosphere chemistry and composition; im- } \\
\text { pacts temperature structure and radiative forcing. }\end{array}$ \\
\hline Solar energetic particles. & $\begin{array}{l}\text { Ionisation of upper and middle atmosphere; impact } \\
\text { on composition and temperatures. Magnetosphere } \\
\text { - ionosphere - thermosphere coupling. }\end{array}$ \\
\hline Galactic cosmic rays & $\begin{array}{l}\text { Ionisation of lower atmosphere; impact on electric } \\
\text { field. Impact on condensation nuclei. }\end{array}$ \\
\hline
\end{tabular}

First, Section 2 considers how information on past climates is derived and look at some of the (mainly circumstantial) evidence that variations in solar activity have affected climate on a wide range of timescales. 


\section{Climate Records}

\subsection{Measurements and reconstructions}

Assessment of climate variability and climate change depends crucially on the existence and accuracy of records of meteorological parameters. Ideally records would consist of long time series of measurements made by well-calibrated instruments located with high density across the globe. In practice, of course, this ideal cannot be met. Measurements with global coverage have only been made since the start of the satellite era about 25 years ago while reliable instrumental records, covering the past few centuries, are only available from a few locations, mainly in Europe. For longer periods, and in remote regions, records have to be reconstructed from indirect indicators of climate known as proxy data. A readable review of the methods involved in the accumulation of climate data, and in its interpretation, is provided by Burroughs (1992).

The longest homogeneous instrument-based temperature series in the world is the Central England Temperature record dating back to 1659. It was first constructed in the 1970s from an accumulation of measurements made by amateur meteorologists in central lowland England. The construction of a homogeneous record requires knowledge of how, and at what time of day, the measurements were made and how local conditions may account for regional variations. Other similar temperature records dating back to the mid 18th century are available for Munich, Vienna, Berlin and Paris among other sites in Europe and at least one in the Eastern United States. These datasets have been extensively analysed for periodic variations in temperature. Generally they show clear indications of variations on timescales of about 2.2 to 2.4 years and 2.9 to 3.9 years but on longer timescales individual records show peaks at different frequencies with little statistical significance.

The most complete record of rainfall comes from eastern China where careful observations of floods and droughts date back to the fifteenth century. Again spectral analysis results in periodicities which vary from place to place.

Figure 1 shows instrumental measurements of surface temperature compiled to produce a global average dating back to 1860. Much of the current concern with regard to global warming stems from the obvious rise over the twentieth century and a key concern of contemporary climate science is to attribute cause(s) to this warming. This is discussed further in Section 3.

Other climate records suggesting that the climate has been changing over the past century include the retreat of mountain glaciers, sea level rise, thinner Arctic ice sheets and an increased frequency of extreme precipitation events (IPCC, 2007).

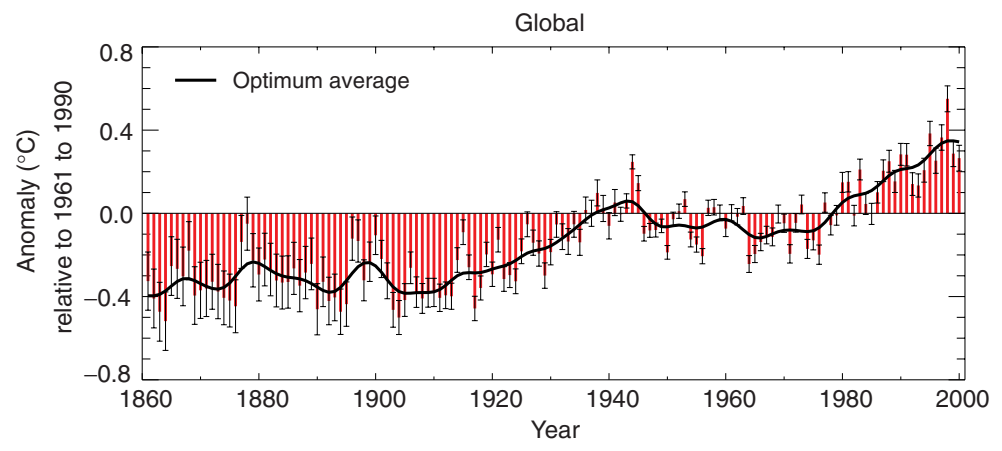

Figure 1: Variations in the global annual average surface temperature over 140 years from instrumental records. From IPCC (2001).

Proxy data provide information about weather conditions at a particular location through

Living Reviews in Solar Physics

http: //www . livingreviews . org/lrsp-2007-2 
records of a physical, biological or chemical response to these conditions. Some proxy datasets provide information dating back hundreds of thousands of years which make them particularly suitable for analysing long term climate variations and their correlation with solar activity.

One well established technique for providing proxy climate data is dendrochronology, or the study of climate changes by comparing the successive annual growth rings of trees (living or dead). It has been found that trees from any particular area show the same pattern of broad and narrow rings corresponding to the weather conditions under which they grew each year. Thus samples from old trees can be used to give a time series of these conditions. Felled logs can similarly be used to provide information back to ancient times, providing it is possible to date them. This is usually accomplished by matching overlapping patterns of rings from other trees. Another problem that arises with the interpretation of tree rings is that the annual growth of rings depends on a number of meteorological variables integrated over more than a year so that the dominant factor determining growth varies with location and type of tree. At high latitudes the major controlling factor is likely to be summer temperature but at lower latitudes humidity may play a greater role. Figure 2 shows a 1000-year surface temperature record reconstructed from proxy data, including tree rings. It shows that current temperatures are higher than they have been for at least the past millennium.

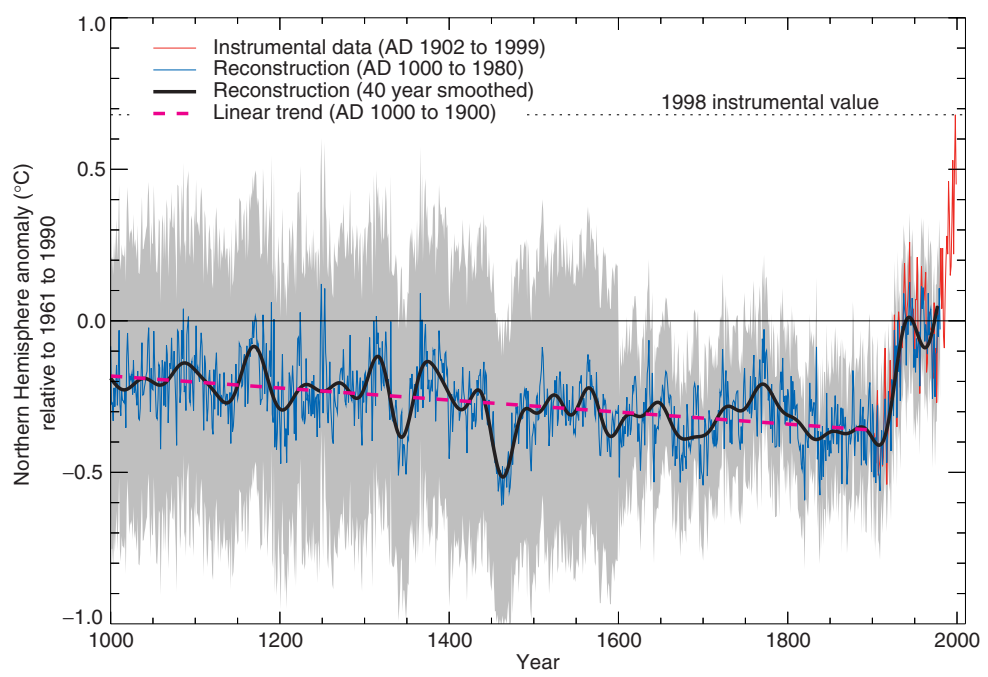

Figure 2: Variations in Northern Hemisphere surface temperature over the past millennium from proxy records (tree rings, corals, ice cores). From IPCC (2001).

Much longer records of temperature have been derived from analysis of oxygen isotopes in ice cores obtained from Greenland and Antarctica. The ratio of the concentration of ${ }^{18} \mathrm{O}$ to that of ${ }^{16} \mathrm{O}$, or ${ }^{2} \mathrm{H}$ to ${ }^{1} \mathrm{H}$, in the water molecules is determined by the rate of evaporation of water from tropical oceans and also the rate of precipitation of snow over the polar ice caps. Both these factors are dependent on temperature such that greater proportions of the heavy isotopes are deposited during periods of higher global temperatures. As each year's accumulation of snow settles the layers below become compacted so that at depths corresponding to an age of more than 800 years it becomes difficult to precisely date the layers. Nevertheless, variations on timescales of more than a decade have been extracted dating back over hundreds of thousands of years.

Figure 3 shows the temperature record deduced from the deuterium ratio in an ice core retrieved from Vostok in East Antarctica. The roughly 100,000 year periodicity of the transitions from glacial to warm epochs is clear and suggests a relationship with the variations in eccentricity of 
the Earth's orbit around the Sun (see Section 4.1) although this does not explain the apparently sharp transitions from cold to warm periods.

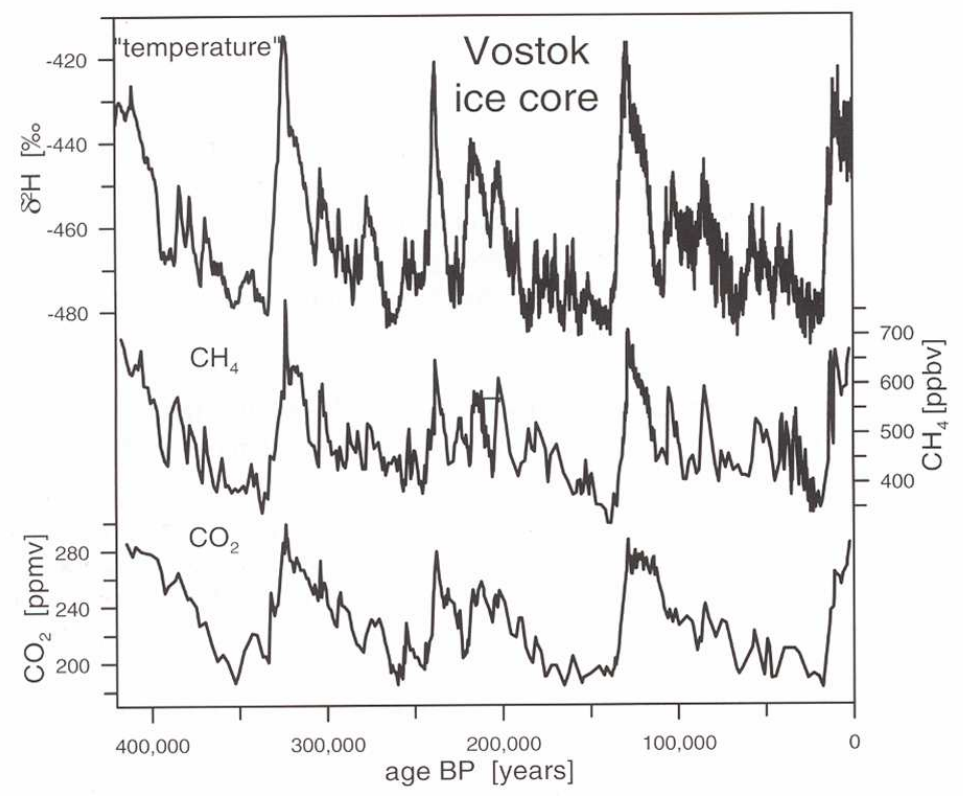

Figure 3: Records derived from an ice core taken from Vostok, East Antarctica, showing variations in temperature (derived from deuterium measurements) and the concentrations of methane and carbon dioxide over at least 400,000 years. From Stauffer (2000).

Evidence of very long term temperature variations can also be obtained from ocean sediments. The skeletons of calciferous plankton make up a large proportion of the sediments at the bottom of the deep oceans and the ${ }^{18} \mathrm{O}$ component is determined by the temperature of the upper ocean at the date when the living plankton absorbed carbon dioxide. The sediment accumulates slowly, at a rate of perhaps $1 \mathrm{~m}$ every 40,000 years, so that changes over periods of less than about 1,000 years are not detectable but ice age cycles every 100,000 years are clearly portrayed.

Ocean sediments have also been used to reveal a history of temperature in the North Atlantic by analysis of the minerals believed to have been deposited by drift ice (Bond et al., 2001). In colder climates the rafted ice propagates further south where it melts, depositing the minerals. An example of such an analysis is presented in Figure 4 and discussed in Section 2.2.

\subsection{Solar signals in surface climate}

Many different approaches have been adopted in the attempt to identify solar signals in climate records. Probably the simplest has been the type of spectral analysis mentioned above, in which cycles of 11 (or 22 or 90 etc.) years are assumed to be associated with the Sun. In another approach time series of observational data are correlated with time series of solar activity. An extension of the latter method uses simple linear regression to extract the response in the measured parameter to a chosen solar activity forcing. A further development allows a multiple regression, in which the responses to other factors are simultaneously extracted along with the solar influence. More sophisticated statistical techniques, involving e.g. pattern-matching, have also been employed. Each of these approaches gives more faith than the previous that the signal extracted is actually due to the Sun, and not to either some other factor or to random fluctuations in the climate system, and

Living Reviews in Solar Physics

http://www. livingreviews.org/lrsp-2007-2 
many interesting results emerge. It should be remembered, however, that such detection, while potentially robust in statistical terms, is not based on any understanding of how the presumed solar influence takes place. In the remainder of this section some results are presented from studies involving correlations and regressions of meteorological data with solar activity indices. Some of the mechanisms which have been proposed to explain how these changes take place are the subjects of Sections 3 to 7.

Section 2.1 mentioned how temperature records may be extracted from ice cores and ocean sediments. These media may also preserve information on cosmic ray flux, and thus solar activity, in isotopes such as ${ }^{10} \mathrm{Be}$ and ${ }^{14} \mathrm{C}$. Thus simultaneous records of climate and solar activity may be retrieved. An example is given in Figure 4 which shows fluctuations on the 1,000 year timescale well correlated between the two records, suggesting a long-term solar influence on climate.

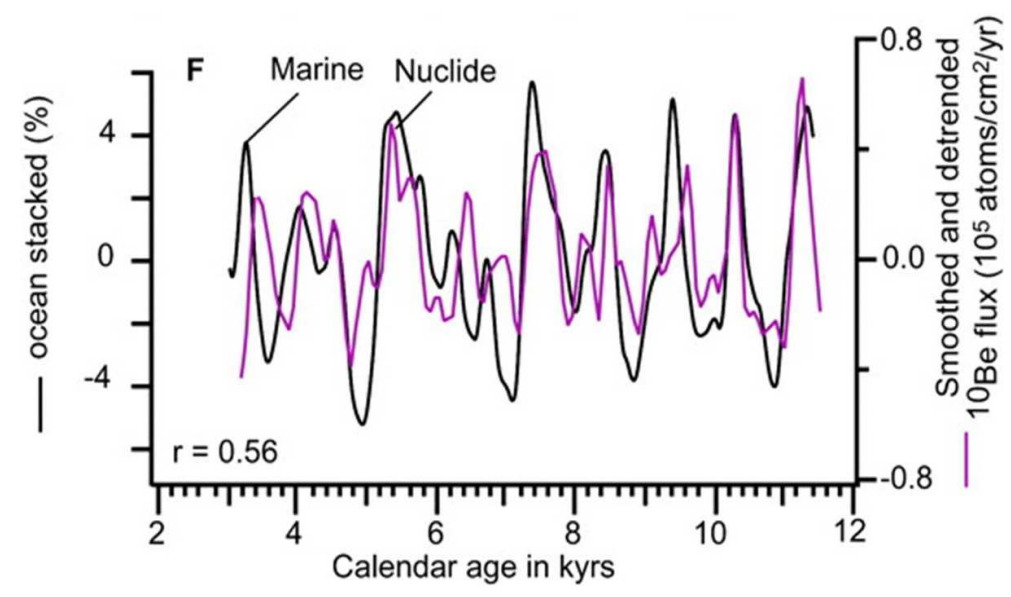

Figure 4: Records of ${ }^{10}$ Be and ice-rafted minerals extracted from ocean sediments in the North Atlantic. From Bond et al. (2001).

On somewhat shorter timescales it has frequently been remarked that the Maunder Minimum in sunspot numbers in the second half of the seventeenth century coincided with what has become known as the "Little Ice Age" during which western Europe experienced significantly cooler temperatures. Figure 5 shows this in terms of winter temperatures measured in London and Paris compared with the ${ }^{14} \mathrm{C}$ ratio found in tree rings for the same dates. Similar cooling has not, however, been found in temperature records for the same period across the globe which (if this signal is reliable) suggests that the Sun's influence may be geographically non-uniform.

A paper published by Friis-Christensen and Lassen (1991) caused considerable interest when it appeared to show that temperature variations over the observational period could be ascribed entirely to solar variability. The measure of solar activity used was the length of the solar cycle (SCL) and, as can be seen in Figure 6, this value coincides almost exactly with the Northern Hemisphere land surface temperature record. The SCL values were, however, smoothed with a (1, $2,2,2,1)$ running filter so that at each point the value given has contributions from four solar cycles both forwards and back in time, i.e. a total of $\sim 50$ years. This means that the values given for dates more recent than about 1958 required some extrapolation, and the more recent the more the result depended on assumptions about future behaviour. Thus the upturn in SCL values in the latter part of the twentieth century was essentially construed. This work has been repeated by Laut and Gundermann (2000), using longer records, as shown in Figure 7. There is still a correspondence between the two records in the first half of the twentieth century but the potential for the Sun to be the cause of more recent warming looks far less convincing. The detection and 


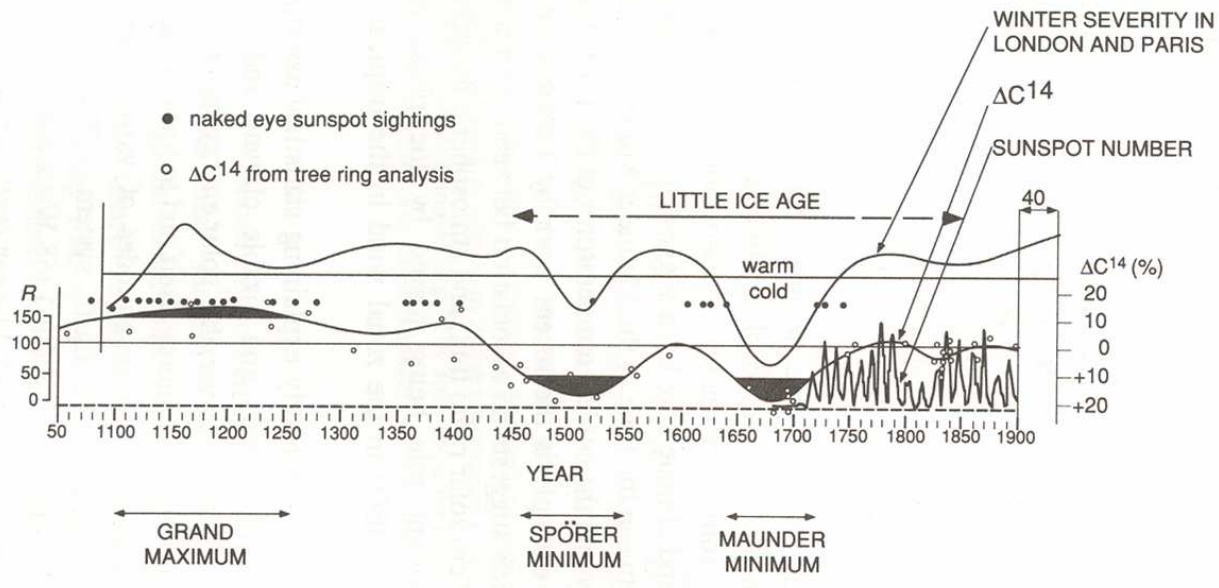

Figure 5: From a paper by Eddy (1976) suggesting that winter temperatures in NW Europe are correlated with solar activity. Note the coincidence of the "Little Ice Age" with the Maunder Minimum in sunspots.

attribution of the causes of twentieth century climate change is discussed further in Section 6.1.

Many studies have purported to show variations in meteorological parameters in phase with the "11-year" solar activity cycle. Some of these are statistically questionable and some show signals that appear over a certain period only to disappear, or even reverse, over another period. The evidence of a solar influence on climate on solar cycle timescales is, however, becoming increasingly robust.

Solar signals have been detected in sea surface temperatures (SSTs). Figure 8 presents some results from an EOF analysis of upper ocean temperatures, from bathythermographs 1955-1994, with the upper panel showing the time varying amplitude of the pattern of response shown in the lower panel. Two interesting features emerge from this work, one is that SSTs do not warm uniformly in response to enhanced solar activity: indeed, the pattern shows latitudinal bands of warming and cooling, and secondly that the amplitude of the change is larger than would be predicted by radiative considerations alone, given the known variations in TSI over the same period (see Section 3.2).

An analysis of a different SST dataset, dating back to the mid-19th century, is shown for the Pacific Ocean in Figure 9. A composite of data from peak years of solar activity cycles has been constructed and the composite of the remaining years subtracted. The results also suggest considerable geographical variation with a mid-latitude pattern resembling the negative phase of the Pacific Decadal Oscillation (PDO) and a tropical signal similar to the negative phase of the El Niño-Southern Oscillation (ENSO). However, the signals derived in Figure 8 and Figure 9 appear to be in the opposite sense and it remains to be seen which, if either, is a true response to the Sun.

These results are suggestive that the Sun may exert some influence on the phases of various climate "modes of variability". Of these ENSO dominates the tropics and the PDO the North Pacific. The North Atlantic exhibits an oscillation in surface pressure referred to as the North Atlantic Oscillation (NAO) which has a strong influence on the climate of Western and Northern Europe. Correlations have been demonstrated between the NAO and the electric field strength of the solar wind (Boberg and Lundstedt, 2002) and between the NAO and solar $10.7 \mathrm{~cm}$ radio flux (Haigh and Roscoe, 2006). These studies imply that Western Europe experiences warmer and wetter winter weather when the Sun is more active, with more and stronger storms crossing

Living Reviews in Solar Physics

http://www. livingreviews.org/lrsp-2007-2 


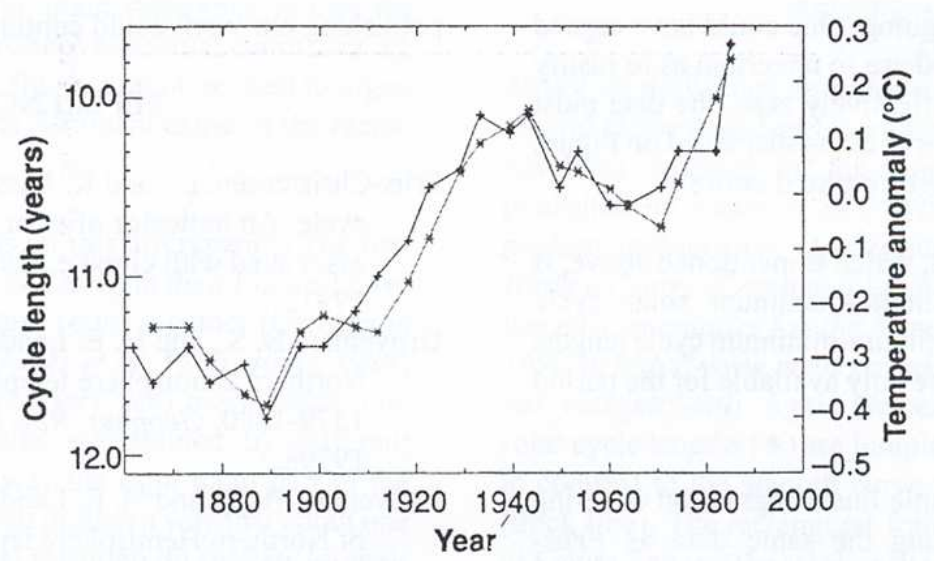

Figure 6: Northern Hemisphere land temperature, (stars) and solar cycle length (inverted, pluses). Both time series are smoothed using (12221) filter weightings. From Friis-Christensen and Lassen (1991).

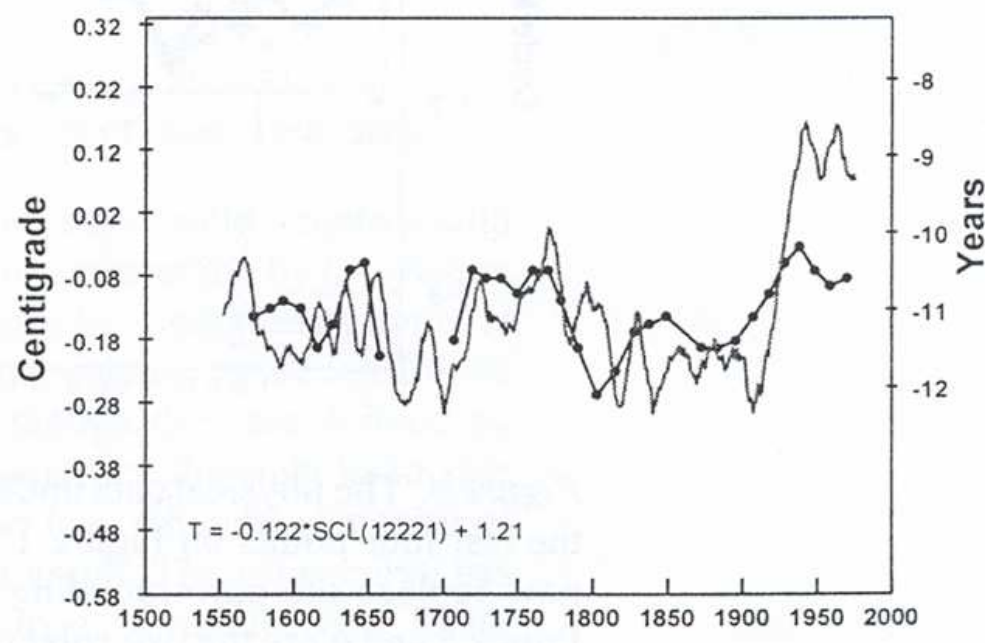

Figure 7: Northern Hemisphere temperatures (Mann et al., 1999) (thin line) and solar cycle length (inverted, dots and thick line), smoothed as in Figure 6. From Laut and Gundermann (2000). 


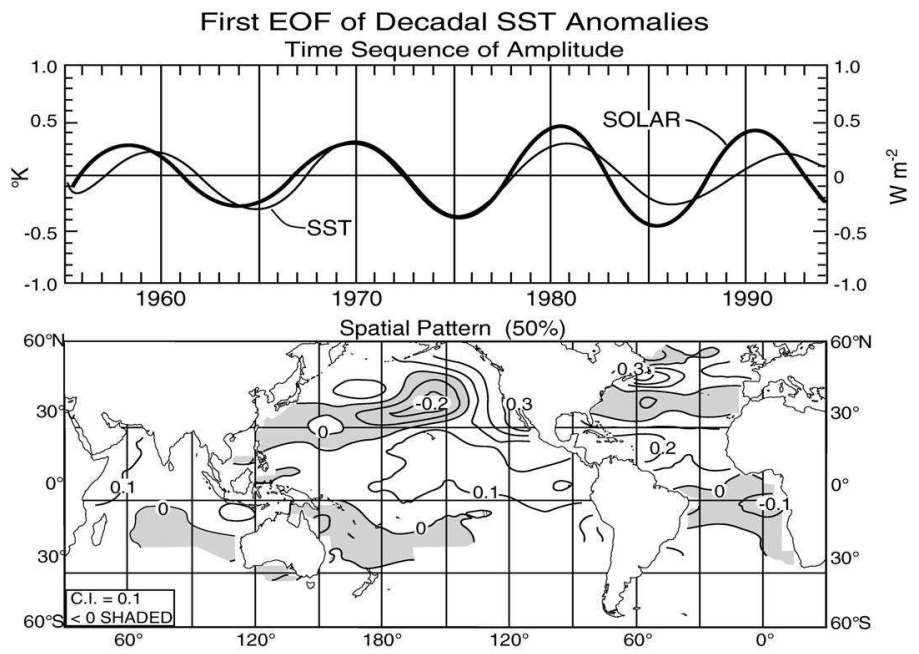

Figure 8: Below: pattern of response in sea surface temperatures derived for solar variability. Above: time series of the magnitude of this pattern. From White et al. (1997).

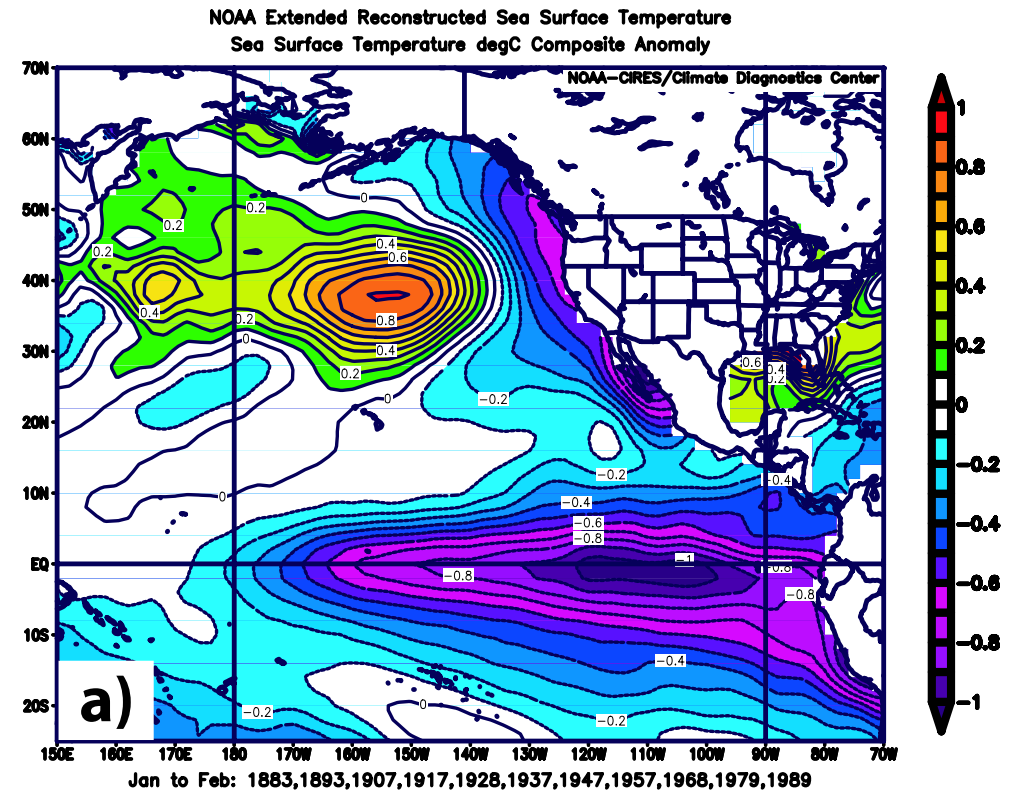

Figure 9: Anomalies of sea surface temperature in peak years of the solar cycle. From van Loon et al. (2007). 
the Atlantic on a more northerly track. For the Northern and Southern Annular Modes (NAM and SAM, representing the main modes of variability in high latitudes) Haigh and Roscoe (2006) found that the combined influence of the Sun and the Quasi-Biennial Oscillation (QBO) of tropical stratospheric winds provided a better indicator, than solar influence alone, of the strength of the circumpolar circulation in both hemispheres (see Table 2). Further discussion of links with the QBO and possible reasons for these are presented in Sections 2.3 and 6.2 respectively.

Table 2: Multiple regression analysis of the surface Northern Annular Mode (NAM) (in winter) and Southern Annular Mode (SAM). The annular modes are the first empirical orthogonal functions of 90-day low-pass filtered anomalies, poleward of $20^{\circ}$ in each hemisphere, of $1000 \mathrm{hPa}$ geopotential height in normalised units. Positive values indicate a stronger equator-to-pole temperature gradient and more zonal flow. Columns show regression coefficients for linear trend (N.H. only), stratospheric chlorine (S.H. only), El Niño-Southern oscillation (ENSO), volcanic (stratospheric) aerosol loading, solar variability (10.7 cm index) and the Quasi-Biennial Oscillation (QBO). Sol*QBO indicates that an index composed of a product of the solar and QBO indices was used in place of those two factors individually. The data cover the period 1958-2001. Colours indicate the statistical significance levels of the values, derived using a Student's t-test: 99\%, 95\%, 90\%, 80\%, <80\%. From Haigh and Roscoe (2006).

\begin{tabular}{|l|c|c|c|c|c|c|c|}
\hline & Trend & Cl & ENSO & Vol & Sol & QBO & Sol* QBO $^{*}$ Q \\
\hline NAM (DJFM) & 0.23 & & -0.82 & 1.08 & 0.81 & 0.64 & \\
& 0.24 & & -0.85 & 0.96 & & & -1.16 \\
SAM & & 1.01 & -0.82 & -0.65 & -0.04 & 0.13 & \\
& & 1.01 & -0.71 & -0.75 & & & -0.89 \\
\hline
\end{tabular}

Another study of correlations between solar activity and climate was carried out by Marsh and Svensmark (2000) who showed that between 1983 and 1994 low latitude low cloud cover varied in phase with galactic cosmic ray (GCR) intensity. An update to this (see Figure 10) found the correlation continued but only if assumptions where made with regard to temporal variations in the calibration of the satellite data used in constructing the cloud dataset. These have still to be validated. Physical mechanisms which might induce a causal relationship between cloud cover and cosmic rays are discussed in Section 7 but it is worth noting here that, if there is a link between cloud and solar activity, then this result alone does not show that it is due to GCRs any more than any other solar- modulated input to the Earth. Indeed, work by Kristjansson and Kristiansen (2000) suggests that there is a stronger correlation between cloud cover and solar UV radiation.

\subsection{Solar signals throughout the atmosphere}

The pioneering work of Karin Labitzke demonstrated a solar cycle variation in stratospheric temperatures. An example of her work is presented in Figure 11 which shows the annual mean, at a location near Hawaii in the sub-tropical Pacific Ocean, of the geopotential height of the $30 \mathrm{hPa}$ pressure surface. This is a measure of the mean temperature of the atmosphere below about $24 \mathrm{~km}$ altitude. It varies in phase with the solar $10.7 \mathrm{~cm}$ radio wave flux over three and a half solar cycles with an amplitude suggesting that the lower atmosphere is $0.5-1.0 \mathrm{~K}$ warmer at solar maximum than at solar minimum. This is a large response but from this figure alone it is not clear whether it applies locally or globally or how the temperature anomaly is distributed in the vertical.

Figure 12 shows the mean summer time temperature of the upper troposphere (between about 2.5 and $10 \mathrm{~km}$ altitude) averaged over the whole Northern Hemisphere. Again this parameter varies in phase with the solar $10.7 \mathrm{~cm}$ index suggesting that the signal seen at the sub-tropical 


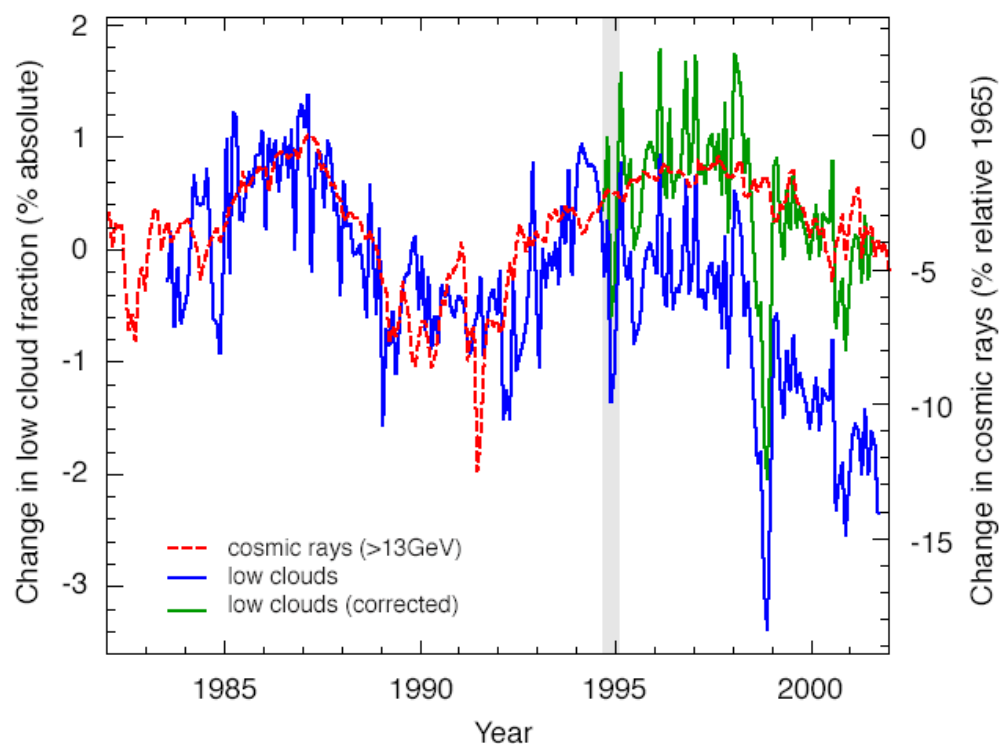

Figure 10: Variation of low cloud cover (ISCCP-D2 data) and cosmic rays between 1984 and 2002. The green curve shows data obtained by applying assumed satellite recalibrations. Redrawn from Marsh and Svensmark (2003) by Gray et al. (2005).

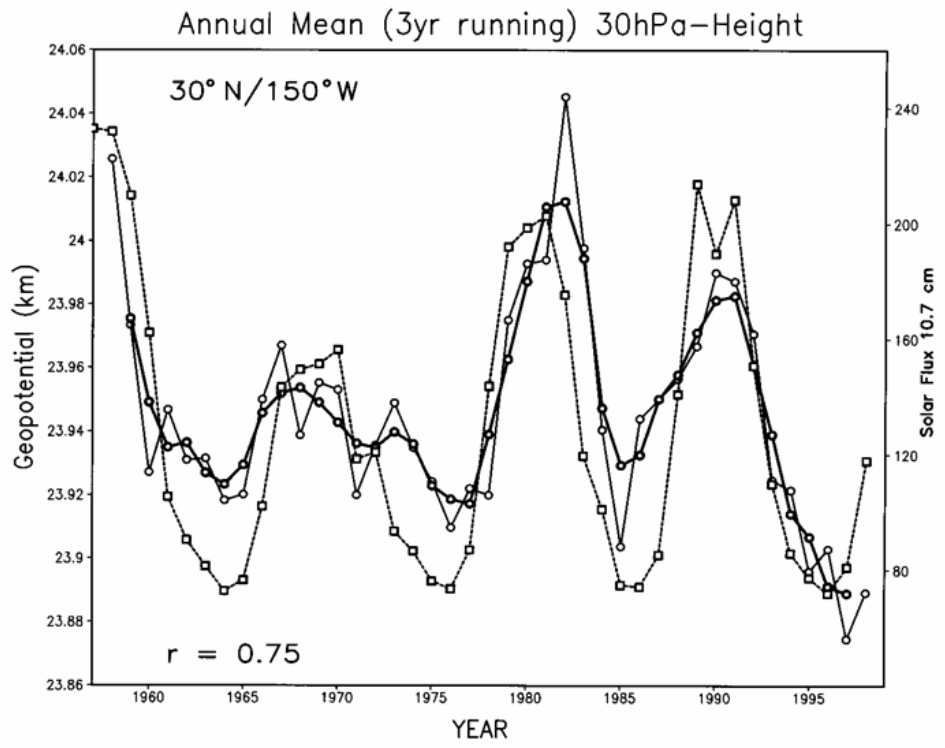

Figure 11: Time series of annual mean $30 \mathrm{hPa}$ geopotential height (km) at $30^{\circ} \mathrm{N}, 150^{\circ} \mathrm{W}$ (thin line with circles), its three-year running mean (thick line with circles) and the solar $10.7 \mathrm{~cm}$ flux (dashed line with squares). From Labitzke and van Loon (1995). 
station of Figure 11 is not confined to that location. The hemispherically-averaged signal, however, has a somewhat smaller amplitude at $0.2-0.4 \mathrm{~K}$.

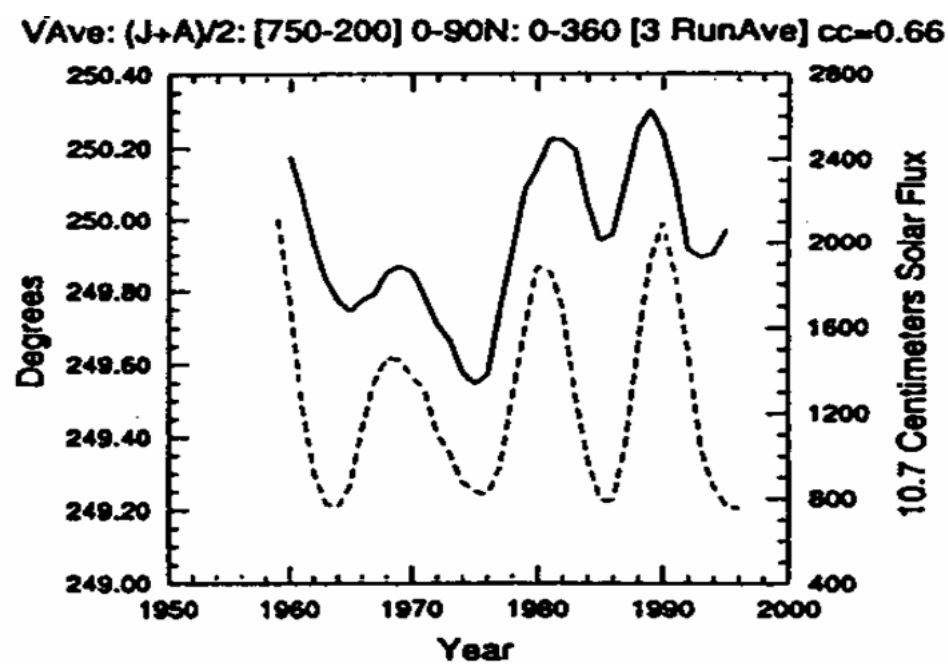

Figure 12: Time series of the mean temperature $(K)$ of the 750-200 hPa layer in the northern hemisphere summer (solid line) and the solar $10.7 \mathrm{~cm}$ flux $\left(10^{-21} \mathrm{Wm}^{-2} \mathrm{~Hz}^{-1}\right)$ (dashed line). From van Loon and Shea (1999).

Figure 13 shows the results of a multiple regression analysis of zonal mean temperatures from the NCEP/NCAR reanalysed dataset (acquired from the Climate Diagnostics Center, Boulder, Colorado, U.S.A., at http: //www.cdc.noaa.gov/). In this work data for 1978-2002 were analysed simultaneously for ten signals: a linear trend, ENSO, NAO, solar activity, volcanic eruptions, QBO and the amplitude and phase of the annual and semi-annual cycles. It is assumed that all these factors are independent and, indeed, the patterns of response for each signal are found to be statistically significant and separable from the other patterns, however it means that any potential solar influence on the NAO (as discussed in Section 2.2) cannot be resolved. There is a linear trend of warming in the troposphere and cooling in the stratosphere, as expected from enhanced concentrations of greenhouse gases; there is a strong ENSO signal in the tropical troposphere but it is also seen in mid-latitudes and throughout the lower stratosphere. The solar response shows largest warming in the tropical stratosphere and in bands of warming, of $>0.4 \mathrm{~K}$, throughout the troposphere in mid-latitudes. The bands of warming/cooling near the surface are consistent with the sea surface temperature study of White et al. (1997), (see Section 2.2) which was based on an entirely different dataset. Note that the analysis was carried out independently at each grid-point so that the hemispheric symmetry of the solar signal provides support for the validity of this result.

In the middle atmosphere measurements made from satellites suggest an increase of up to about $1 \mathrm{~K}$ in the upper stratosphere at solar maximum and of a few tenths of a degree in the lower stratosphere, but a minimum, possibly even negative, response in between. However, precise values, as well as the position (or existence) of the negative layer, vary between datasets: some examples are given in Figure 14.

The Quasi-Biennial Oscillation (QBO) is a naturally-occurring variation in zonal winds in the tropical lower stratosphere which has a period varying around 26 months. Accepted theory has it that a west phase in the tropical QBO is linked with cold temperatures in the winter polar lower stratosphere, with vertically propagating planetary waves being channelled equatorwards. Karin Labitzke has pointed out, however, that while this relationship holds well during periods of lower solar activity it tends to break down near solar maximum. Figure 15, which contains data for the 

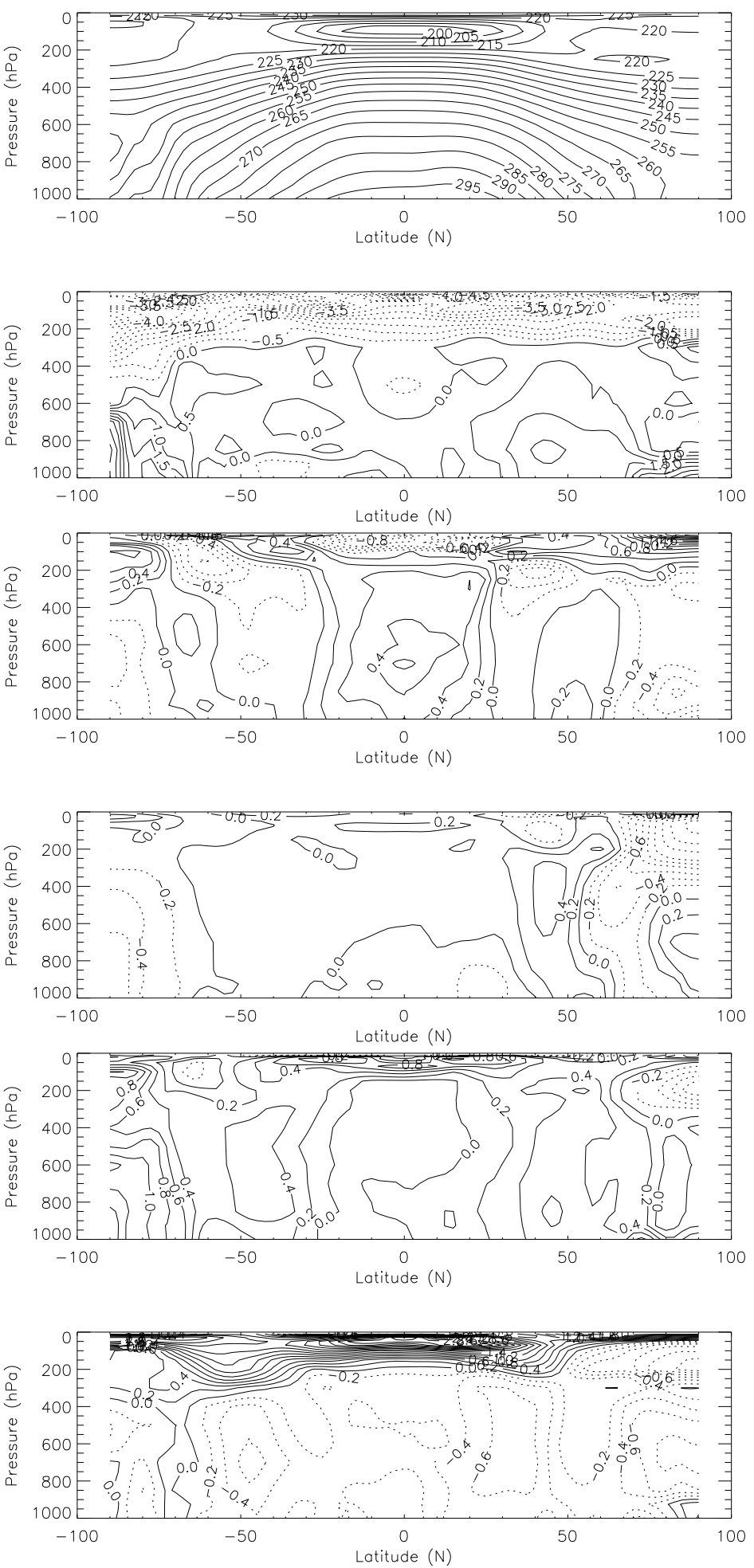

Figure 13: Results from multiple regression analysis of NCEP zonal mean temperatures and zonal winds. From top: mean temperature; linear trend ( $K$ over 44 years); ENSO (max-min, K); NAO (max-min, K), solar cycle (max-min, K); volcanic eruption (effect of Mt. Pinatubo eruption, K) From Haigh (2003). 

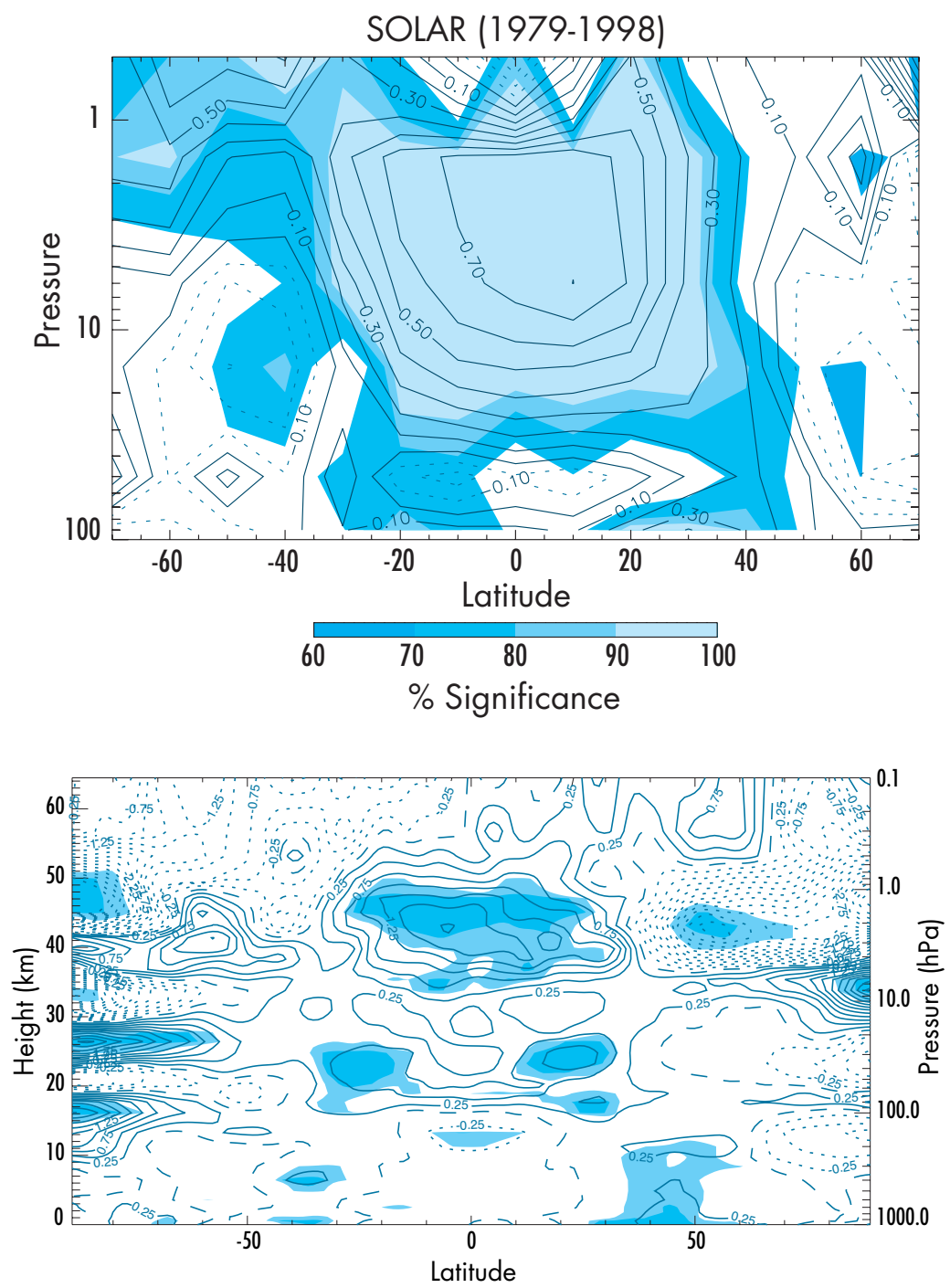

Figure 14: Difference in annual mean temperature between solar maximum and solar minimum derived from observational data. Above: SSU/MSU satellite data (grey shading denotes statistical significance as shown in the legend). Below: ERA reanalysis data for the period 1979-2001; light/dark shading denotes $95 \%$ and $99 \%$ significance. Note the different height ranges in the two panels. From Haigh et al. (2004). 
years 1956-1991, shows that warm polar temperatures tend to occur during the east phase of the QBO at solar minimum and west phase at solar maximum. The reason for this is an active area of current research in dynamical meteorology and is discussed below in Section 5.4.

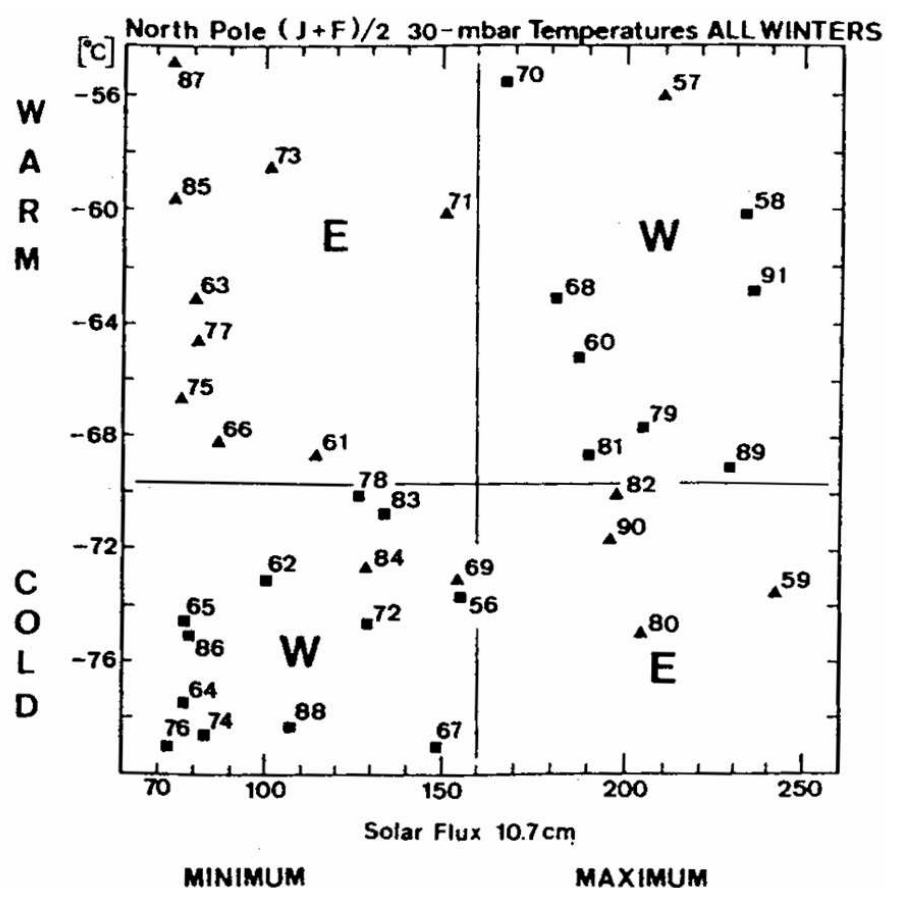

Figure 15: A scatter plot showing for each year values of 30 hPa temperature at the north pole in $\mathrm{Jan} / \mathrm{Feb}$ (ordinate), solar $10.7 \mathrm{~cm}$ flux (abscissa) and phase of the $Q B O$ (symbols, triangle for east and square for west). The horizontal and vertical lines, and the $E \mathcal{E} W$ labels, have been drawn to indicate regions of the diagram in which certain phases of the $Q B O$ predominate. From Labitzke and van Loon (1992).

Another important aspect of the Sun's influence on the stratosphere is in the modulation of ozone concentrations. This is described in Section 5.4.

Figure 16 presents some results from a multiple regression analysis of zonal mean zonal winds, similar to that carried out on temperatures in Figure 13 although only the solar signal is shown here. When the Sun is more active it appears that the mid-latitude jets are weaker and positioned further polewards. This has implications for the positions of the mid-latitude storm tracks and thus provides further evidence for a solar signal in mid-latitude climate.

Studies have also indicated an impact of solar variability on the meridional circulation of the lower atmosphere. Figure 17 suggests that when the Sun is more active the tropical meridional overturning of the atmosphere is somewhat weaker and broader in latitudinal extent. 

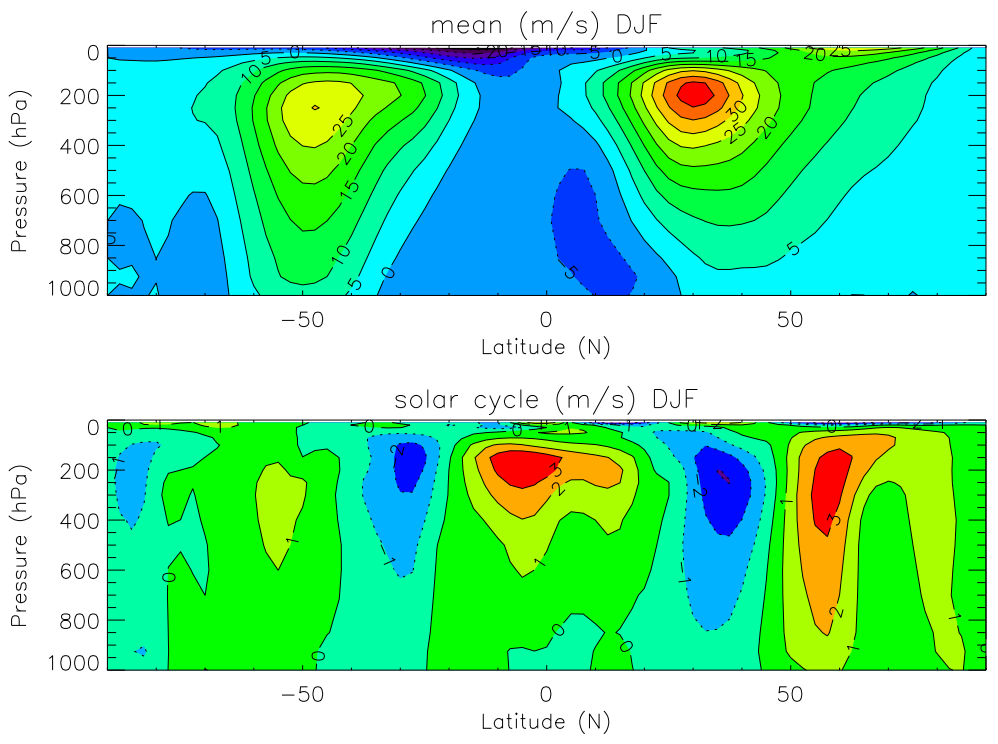

Figure 16: Above: Annual and zonal mean zonal wind, u, as a function of latitude and pressure from NCEP Reanalysis. Below: difference in u between solar maximum and solar minimum. From Haigh et al. (2005).

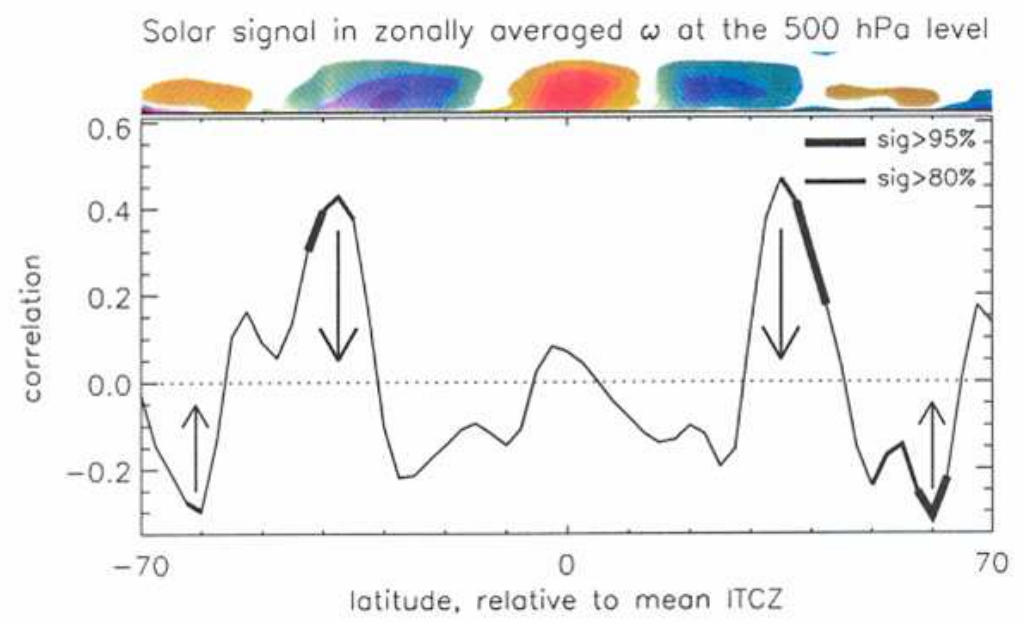

Figure 17: Results from an analysis of NCEP zonal mean vertical (pressure) velocities. The coloured patches indicate the climatological mean at $500 \mathrm{hPa}$ with upwelling $(\omega<0)$ at the equator and sinking in the sub-tropics. The correlation curve shows at solar max positive $\omega$ (i.e. weaker ascent) at the equator and negative/positive values on the equatorward/poleward sides of the descending branches, indicating that these have moved polewards. From Gleisner and Thejll (2003). 


\section{Earth Radiation Budget and Radiative Forcing of Climate Change}

This section introduces the concept of radiative forcing as a tool for estimating the impact on surface temperature of perturbations to the Earth's radiation budget, such as might be due to changes in solar irradiance.

\subsection{Earth radiation budget}

The global average equilibrium temperature of the Earth is determined by a balance between the energy acquired by the absorption of incoming solar radiation and the energy lost to space by the emission of thermal infrared radiation. The amount of solar energy absorbed depends both on the incoming irradiance and on the Earth's reflective properties. If either of these changes then the temperature structure of the atmosphere-surface system tends to adjust to restore the equilibrium. Figure 18 shows the components of the global annual average radiation budget and how much radiation is absorbed, scattered and emitted within the atmosphere and at the surface. The value for the incoming radiation, $342 \mathrm{Wm}^{-2}$, is equivalent to a total solar irradiance at the Earth of $1368 \mathrm{Wm}^{-2}$ averaged over the globe. Of this $31 \%\left(107 \mathrm{Wm}^{-2}\right)$ is reflected back to space by clouds, aerosols, atmospheric molecules and the surface, with the clouds playing the most important role, so that only $235 \mathrm{Wm}^{-2}$ is absorbed by the Earth system. $20 \%\left(67 \mathrm{Wm}^{-2}\right)$ of the incident radiation is absorbed within the atmosphere leaving $49 \%\left(168 \mathrm{Wm}^{-2}\right)$ to reach and heat the surface.

The temperature and emissivity of the surface are such that $390 \mathrm{Wm}^{-2}$ of infrared energy are emitted into the atmosphere. Only $40 \mathrm{Wm}^{-2}$ of this, however, escapes to space with the remainder being absorbed by atmospheric gases and cloud. The atmosphere returns $324 \mathrm{Wm}^{-2}$ to the surface. The energy balance at the surface is achieved by non-radiative processes such as evaporation and convection. The radiation balance at the top of the atmosphere is achieved by the $195 \mathrm{Wm}^{-2}$ of heat energy emitted to space by the atmosphere and clouds.

\subsection{Radiative forcing of climate change}

In equilibrium the net global and annual mean radiative flux at the top of the atmosphere (TOA) is zero. Consider, however, a situation in which some new factor (such as changes in solar irradiance, planetary albedo or the concentrations of radiatively active gases, aerosols or cloud) acts to perturb the absorbed solar or emitted infrared radiation. Then, before surface and atmospheric temperatures have had time to adjust and to establish a new equilibrium, the net flux at TOA is not zero. The simplest definition of radiative forcing $(\mathrm{RF})$ is just the (hypothetical) instantaneous change in the value of the net downward flux. If the RF is positive then there is an increase in energy entering the system (or equivalently a decrease in energy leaving the system) and it will tend to warm until the outgoing energy matches the incoming and the net flux is again zero.

The concept of radiative forcing has been found to be a useful tool in analysing and predicting the response of surface temperature to imposed radiative perturbations. This is because experiments with general circulation models (GCMs) of the coupled atmosphere-ocean system have found that, approximately, the change in globally averaged equilibrium surface temperature, $T_{g}$, is linearly related to the radiative forcing:

$$
\Delta T_{g}=\lambda \mathrm{RF}
$$

where $\lambda$ is the "climate sensitivity parameter". $\lambda$ has been found to be fairly insensitive to the nature of the perturbation and to lie in the range $0.3<\lambda<1.0 \mathrm{~K}\left(\mathrm{Wm}^{-2}\right)^{-1}$. Thus a calculation of the radiative forcing due to a particular perturbant gives a first-order indication of the potential magnitude of its effect on surface temperature without the need for costly GCM runs. Note that

Living Reviews in Solar Physics

http://www. livingreviews.org/lrsp-2007-2 


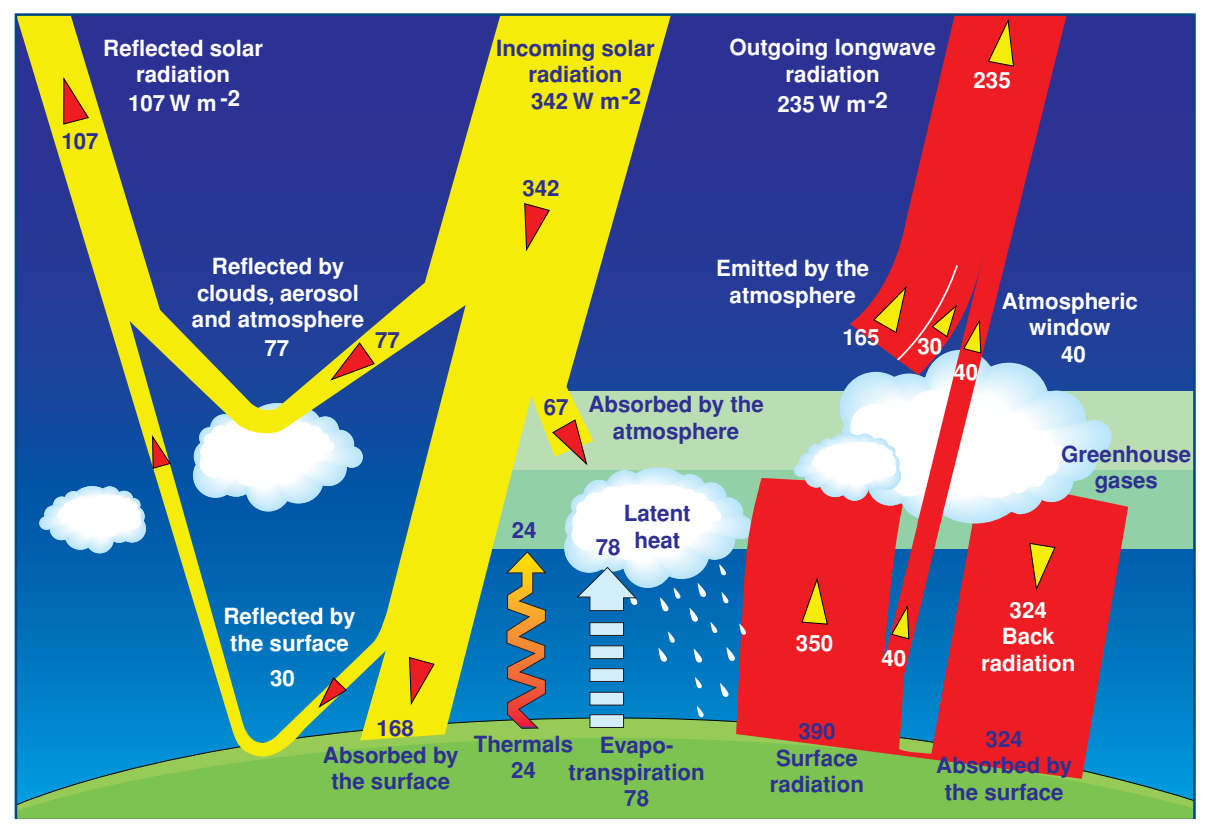

Figure 18: Globally averaged energy budget of the atmosphere. Figure from http://asd-www. larc. nasa. gov/ceres/brochure/ based on data from Kiehl and Trenberth (1997).

incorporated into $\lambda$ are various atmospheric feedback processes. For example, as the climate warms the atmosphere can hold more water vapour; the latter is a strong greenhouse gas and thus increases the initial warming.

The large, factor 3, range in the value of $\lambda$ given above represents the spread of values given by different GCMs. This gives an indication of the uncertainties in climate prediction. It should be noted, however, that for each particular GCM the range of $\lambda$ found using different sources of radiative forcing is much narrower. This suggests that, while absolute predictions are subject to large uncertainty, the forecast of the relative effects of different factors is more reliable.

It has been found that the value of $\lambda$, and thus the usefulness of the radiative forcing concept, is more robust if, instead of using the instantaneous change in net flux at the top of the atmosphere, $\mathrm{RF}$ is defined at the tropopause with the stratosphere first allowed to adjust to the imposed changes. Thus a formal definition of radiative forcing, as used by the Intergovernmental Panel on Climate Change (IPCC) is the change in net flux at the tropopause after allowing stratospheric temperatures to adjust to radiative equilibrium (but with surface and tropospheric temperatures held fixed). The effects of the stratospheric adjustment are complex as can be illustrated by the case of changes in stratospheric ozone: an increase in ozone masks the lower atmosphere from solar ultraviolet, i.e. reducing the net flux at the tropopause and thus RF. However, the presence of ozone in the lower stratosphere increases the downward infrared emission (and thus RF) both directly through the $9.6 \mu \mathrm{m}$ band and also indirectly through the increase in stratospheric temperatures which it produces. Whether the net effect is positive or negative depends on whether the shortwave or longwave effect dominates and this is determined by the vertical distribution of the ozone change.

The direct effect of an increase in total solar irradiance is to increase the radiative forcing. The heating of the stratosphere by the additional irradiance will enhance this by increasing the downward emission of thermal radiation. However, the sign of the radiative forcing due to any solar-induced increases in ozone is not clear - published estimates show both positive and negative values - because of the uncertainties in the distribution of the ozone change (see Section 5.5). 
Figure 19 shows the RF values deduced for the period 1750 to 2005 for a range of different factors. The largest component, $1.66 \mathrm{Wm}^{-2}$, is due to the increase in carbon dioxide with other well-mixed greenhouse gases contributing a further $0.98 \mathrm{Wm}^{-2}$. The other components are all of magnitude a few tenths of a $\mathrm{Wm}^{-2}$. For example, aerosol particles (mainly the result of industrial emissions of sulphur dioxide) have produced a $\mathrm{RF}$ of $-0.5 \mathrm{Wm}^{-2}$, negative because they enhance the planetary albedo. Also shown is a value of $-0.7 \mathrm{Wm}^{-2}$ (with large uncertainty) for the "cloud albedo effect" of aerosol. This represents the process whereby an increase in aerosol concentration produces more cloud condensation nuclei so that clouds tend to be composed of a higher number density of smaller droplets which increases albedo (see Section 7). Note that the error bars in Figure 19 do not represent statistical ranges of uncertainty but just the ranges of values published in peer-reviewed scientific literature. The figure also gives an indication of the "level of scientific understanding" (LOSU) for each effect which is a subjective analysis intended to indicate whether the scientific processes involved were perceived to be complete and well-understood.

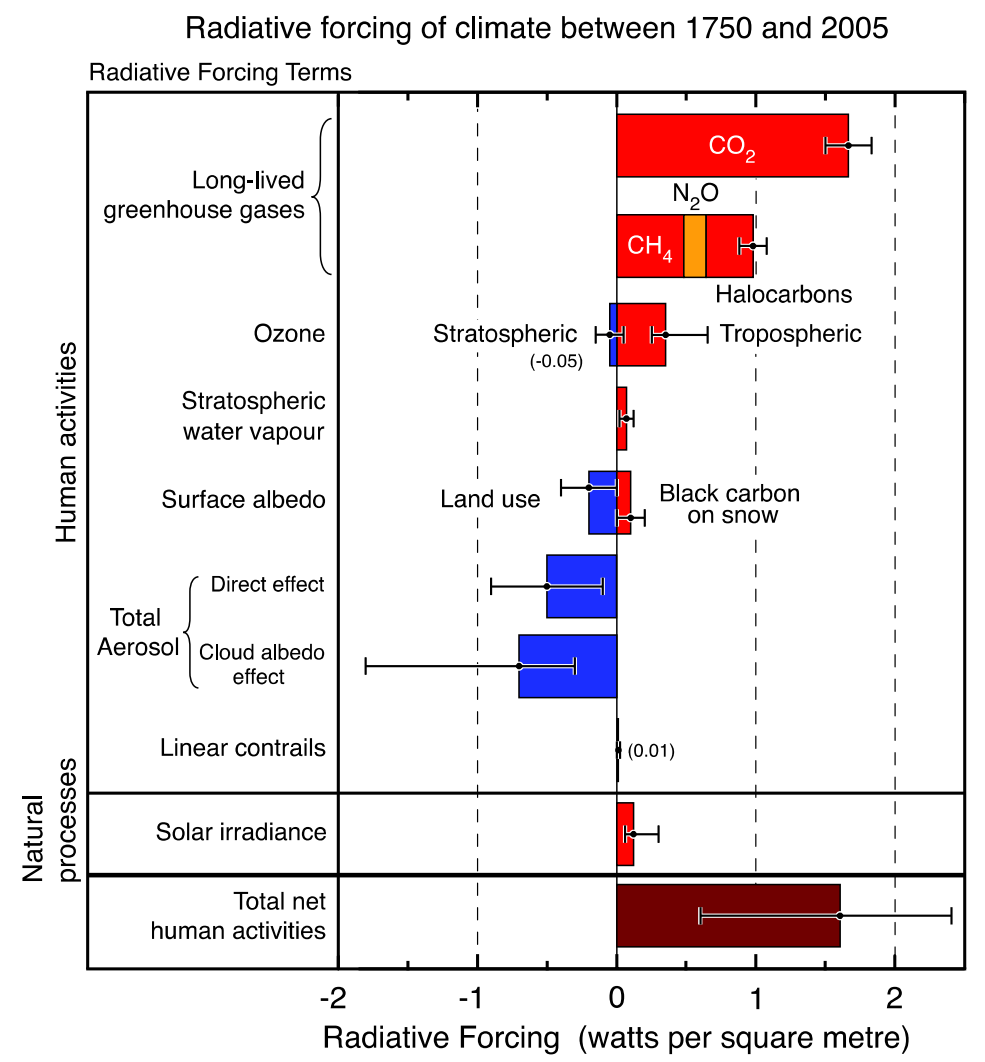

Figure 19: Global, annual average radiative forcing contributions 1750 -2005 from the IPCC fourth assessment report. From IPCC (2007).

The solar contribution is assessed to be in the range $0.06-0.30 \mathrm{Wm}^{-2}$. Note that when calculating solar radiative forcing it is necessary to scale the total solar irradiance at the Earth by a factor taking into account geometric considerations as well as the planetary albedo. Thus the RF due to a change in TSI of $1 \mathrm{Wm}^{-2}$ is about $1 / 6 \mathrm{Wm}^{-2}$, or a change in TSI of $0.7 \mathrm{Wm}^{-2}$ since 1750 is equivalent to $\mathrm{RF}=0.12 \mathrm{Wm}^{-2}$. The actual variations in TSI over the past few centuries is very uncertain (see Section 4.2) and the change in TSI depends crucially on the starting date (chosen 
as 1750 by the IPCC to represent the pre-industrial atmosphere): choice of earlier or later in the 18th century would have given an increased solar RF. Thus the value of solar radiative forcing in the IPCC figure is largely indicative. Taking a value of the climate sensitivity parameter $\lambda$ of $0.6 \mathrm{~K}\left(\mathrm{Wm}^{-2}\right)^{-1}$ suggests that a global $\lambda$ of $0.6 \mathrm{~K}\left(\mathrm{Wm}^{-2}\right)^{-1}$ suggests that a global average surface warming of less than $0.1 \mathrm{~K}$ since 1750 could be ascribed to the Sun. However, the IPCC gives the assignation "very low" to the LOSU associated with solar radiative forcing, thereby acknowledging that there may be factors as yet unknown, or not fully understood, which may act to amplify (or even diminish) its effects. 


\section{Total Solar Irradiance}

\subsection{The Earth's orbit around the Sun}

If the Sun's output were constant then the amount of solar radiation reaching the Earth would depend only on the distance between these two bodies. This distance, $R_{e}$, varies during the year due to the ellipticity of the Earth's orbit which is measured by the value of its eccentricity $(e)$. The value of $e$ itself, however, varies in time with periods of around 100,000 and 413,000 years due to the gravitational influence of the Moon and other planets. At any particular point on the Earth the amount of radiation striking the top of the atmosphere also depends on two other orbital parameters. One of these is the tilt $\left(\theta_{t}\right)$ of the Earth's axis to the plane of its orbit which varies cyclically with a period of about 41,000 years. The other is the longitudinal position of the vernal equinox relative to the perihelion of the orbit $(p)$, which is determined by the precession of the Earth's axis. This varies with periods of about 19,000 and 23,000 years. Figure 20 shows values of $e, \theta_{t}$ and $p$ calculated over a period of several hundred thousand years.

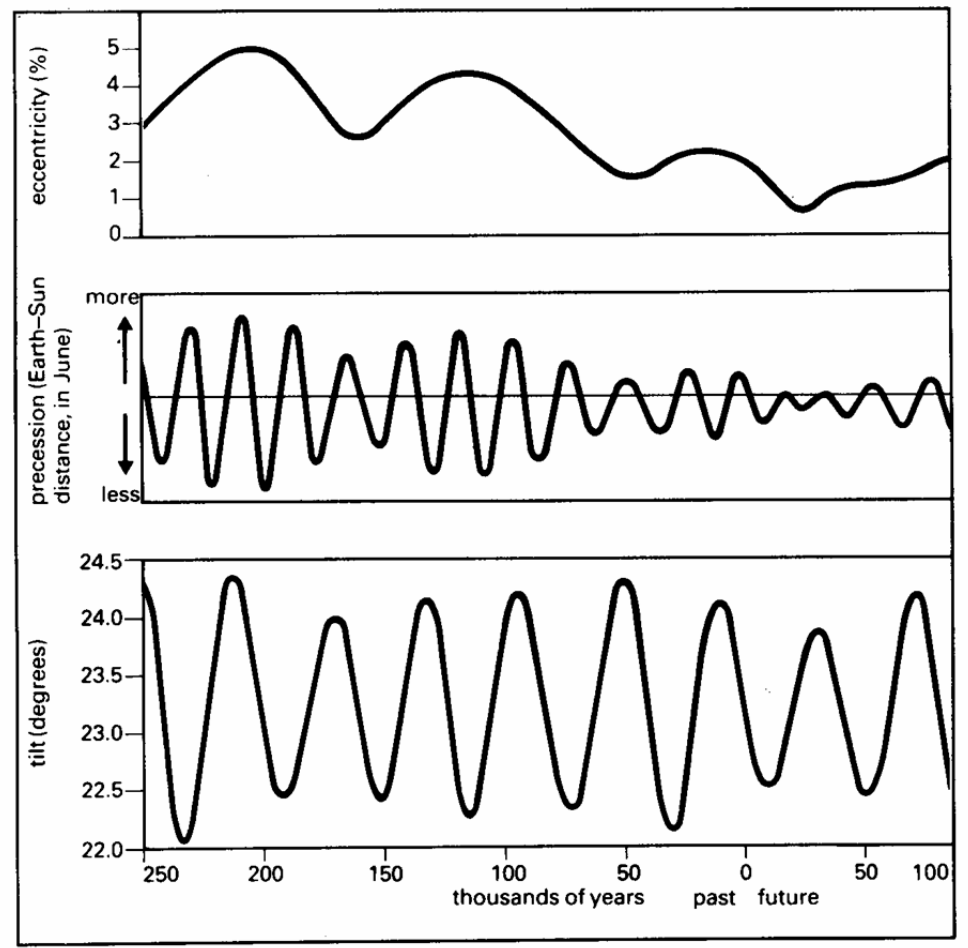

Figure 20: The eccentricity, precession and tilt of the Earth's orbit calculated to take place over 350,000 years. From Burroughs (1992).

Averaged over the globe and over a year the solar energy flux at the Earth depends only on $e$ but seasonal and geographical variations of the irradiance depend on $\theta_{t}$ and $e \sin p$. However, it is not just the temperatures of individual seasons that are at stake: the intensity of radiation received at high northern latitudes in summer determines whether the winter growth of the ice cap will recede or whether the climate will be precipitated into an ice age. Thus changes in seasonal irradiance can lead to much longer term shifts in climatic regime.

Figure 3 showed temperature variations associated with transitions between ice ages and inter-

Living Reviews in Solar Physics

http://www. livingreviews . org/lrsp-2007-2 
glacials but also presented the concentrations of methane and carbon dioxide preserved in the ice core showing a strong correlation between these and temperature. (NB neither concentration reaches anything like the present day values of about $1700 \mathrm{ppbv}$ and $380 \mathrm{ppmv}$ respectively). One theory (Petit et al., 1999) suggests that the warming of southern high latitudes caused by the orbital variations is amplified by the release of $\mathrm{CO}_{2}$ from the southern oceans and this warming is then further amplified through a reduction in albedo resulting from the melting of Northern Hemisphere ice sheets. Such positive feedback mechanisms might explain the sharp increases in temperature seen in the record.

Cyclical variations in climate records with periods of around 19, 23, 41, 100 and $413 \mathrm{kyr}$ are generally referred to as Milankovitch cycles after the geophysicist who made the first detailed investigation of solar-climate links related to orbital variations.

\subsection{Variations in total solar irradiance}

Direct measurements of TSI made outside the Earth's atmosphere began with the launch of satellite instruments in 1978. Previous surface-based measurements did not provide sufficient accuracy, as they were subject to uncertainties and fluctuations in atmospheric absorption that may have swamped the small solar variability signal.

Figure 21a presents all existing satellite measurements of TSI and it is clear that significant uncertainties remain related to the calibration of the instruments and their degradation over time. For example, data from the newest instrument, the Total Irradiance Monitor (TIM) on the SORCE satellite, is giving values approximately $5 \mathrm{Wm}^{-2}$ lower than other contemporaneous instruments which disagree among themselves by a few $\mathrm{Wm}^{-2}$. This uncertainty is a serious problem underlying current solar-climate research. The variation in TSI over the past two 11-year cycles is known to greater accuracy showing approximately $0.08 \%\left(\sim 1.1 \mathrm{Wm}^{-2}\right)$ peak-to-peak over the solar cycle.

There is a related uncertainty, however, in the existence of any underlying trend in TSI over the past 2 cycles. Figure 21b presents one attempt to composite the measurements into a best estimate. It shows essentially no difference in TSI values between the cycle minima occurring in 1986 and 1996. The results of Willson and Mordinov (2003), however, show an increase in irradiance of $0.045 \%$ between these dates. The discrepancy hinges on assumptions made concerning the degradations of the Nimbus7/ERB and ERBS/ERBE instruments, data from which fills the interval, from July 1989 to October 1991, between observations made by the ACRIM I and II instruments. If such a trend were maintained, it would imply an increase in radiative forcing of about $0.1 \mathrm{Wm}^{-2}$ per decade. Compared in terms of climate forcing, this is appreciable, being about one-third that due to the increase in concentrations of greenhouse gases averaged over the past 50 years. These discrepancies are important because all the available TSI reconstructions discussed below either use directly, or are scaled to fit, the recent satellite measurements, using one or other of the TSI composites.

The time interval of satellite observations contains information only on the short term components of solar variability but, in order to assess the potential influence of the Sun on centennialscale climate change, it is necessary to know TSI further back into the past. In reconstructing past changes in TSI, proxy indicators of solar variability, for which longer periods of observation are available, are used to produce an estimate of its temporal variation over the past centuries. There are several different approaches taken to "reconstructing" the TSI, all employing a substantial degree of empiricism, and some examples are given in Figure 22.

It is clear that the estimates diverge as they go back in time so that the value of solar radiative forcing over this period is highly uncertain. Furthermore, this plot does not include some estimates which suggest a much larger difference between the present level and that prevailing during the Maunder Minimum in sunspots which occurred during the latter part of the 17th century (e.g. Hoyt and Schatten, 1998). 


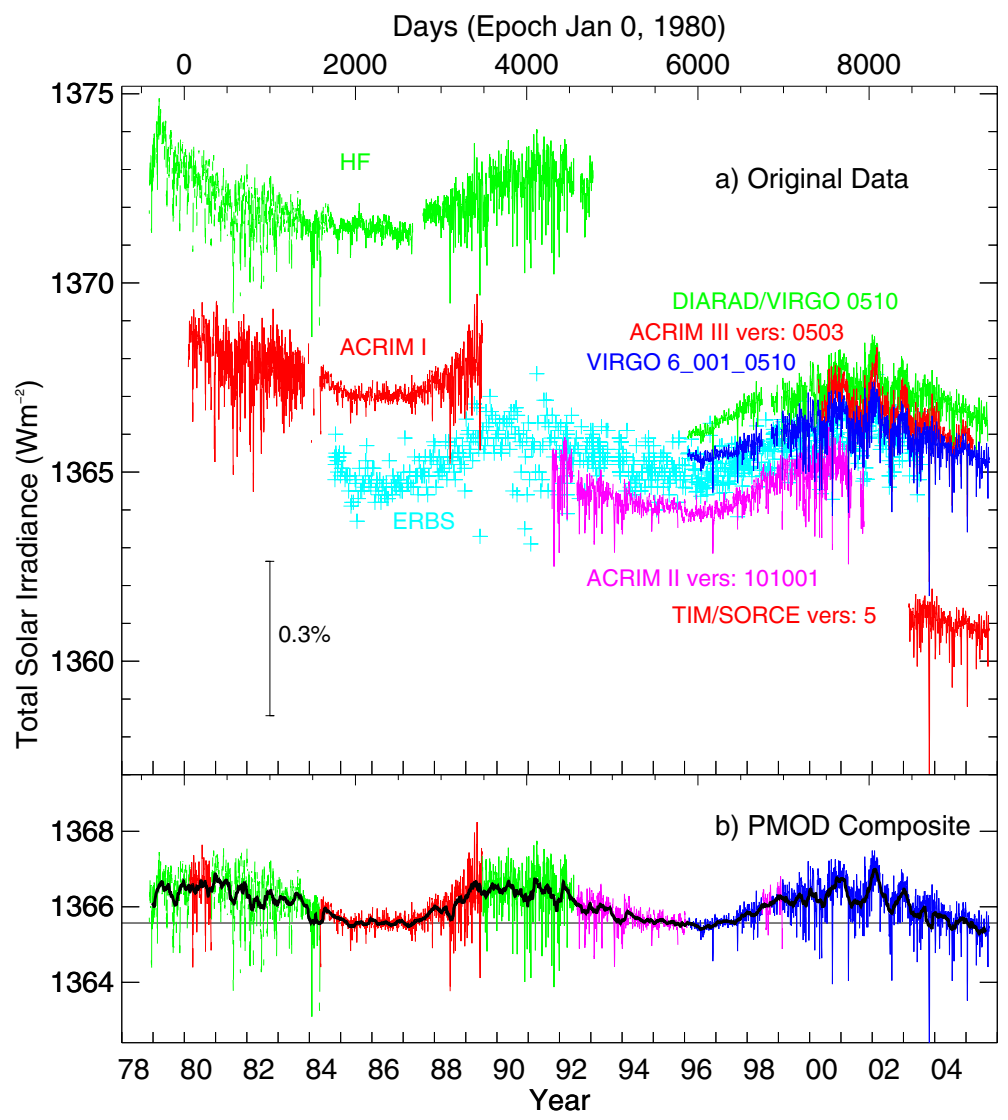

Figure 21: (a) Daily-averaged total solar irradiance: all measurements made from satellites (b) Composite of measurements to produce best estimate of TSI. Figure courtesy of Claus Fröhlich (http : //www. pmodwrc. ch/). 


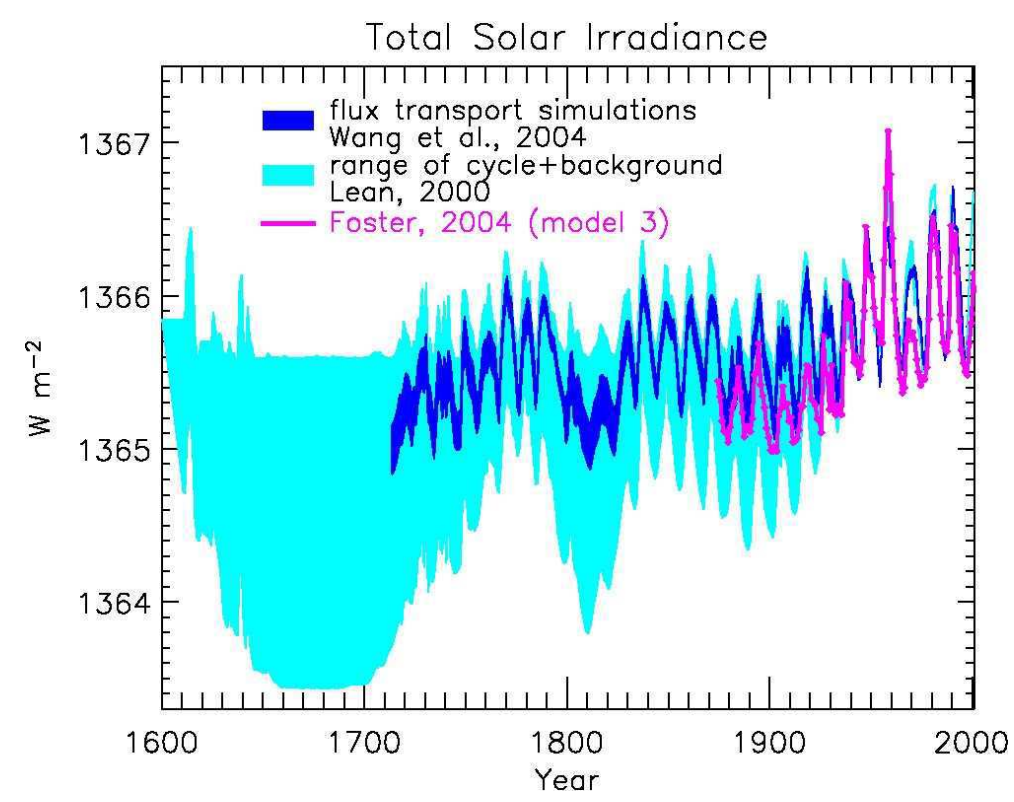

Figure 22: Reconstructions by various authors of total solar irradiance over the past 400 years. From Judith Lean, based on data from Wang et al. (2005); Lean (2000); Foster (2004).

\section{Solar spectral irradiance}

The interaction of solar radiation with the atmosphere is fundamental in determining its temperature structure and in controlling many of the chemical processes which take place there. This section outlines the role of solar UV radiation in determining the budget of stratospheric ozone and discusses how the composition and thermodynamic structure of the stratosphere are modulated by solar variability. It goes on to consider how perturbations to the stratosphere may impact solar radiative forcing of tropospheric climate.

\subsection{Absorption of solar spectral radiation by the atmosphere}

Absorption by the atmosphere of solar radiation depends on the concentrations and spectral properties of the atmospheric constituents. Figure 23 shows a blackbody spectrum at $5750 \mathrm{~K}$, representing solar irradiance at the top of the atmosphere, and a spectrum of atmospheric absorption. Absorption features due to specific gases are clear with molecular oxygen and ozone being the major absorbers in the ultraviolet and visible regions and water vapour and carbon dioxide more important in the near-infrared.

Clearly the flux at any point in the atmosphere depends on the properties and quantity of absorbing gases at higher altitudes and on the path length for the radiation, taking into account the solar zenith angle. The heights at which most absorption takes place at each wavelength can be seen in Figure 24 which shows the altitude of unit optical depth for an overhead Sun. At wavelengths shorter than $100 \mathrm{~nm}$ most radiation is absorbed at altitudes between 100 and $200 \mathrm{~km}$ by atomic and molecular oxygen and nitrogen, mainly resulting in ionized products. Solar Lyman- $\alpha$ radiation at $121.6 \mathrm{~nm}$ penetrates to the upper mesosphere where it makes a significant contribution to heating rates and water vapour photolysis. Between about 80 and $120 \mathrm{~km}$ oxygen is photo-dissociated as it absorbs in the Schumann-Runge continuum between 130 and $175 \mathrm{~nm}$. The Schumann-Runge bands, $175-200 \mathrm{~nm}$, are associated with electronic plus vibrational transitions 


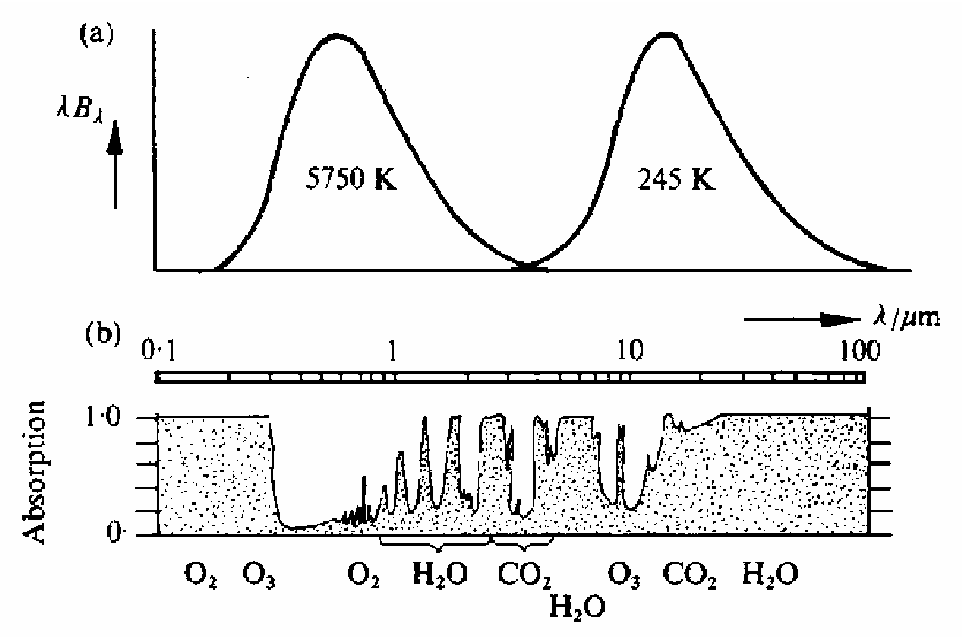

Figure 23: (a) Black body functions at the emitting temperatures of the Sun and the Earth. The functions are scaled to have equal area to represent the Earth's radiation balance. (b) Absorption by a vertical column of atmosphere. The gases responsible for the main absorption features are identified at the appropriate wavelengths. (Houghton, 1977).

of the oxygen molecule and are most significant between 40 and $95 \mathrm{~km}$ altitude. The oxygen Herzberg continuum is found in the range $200-242 \mathrm{~nm}$ and is overlapped by the ozone HartleyHuggins bands between 200 and $350 \mathrm{~nm}$ which are responsible for the photodissociation of ozone below $50 \mathrm{~km}$. The ozone Chappuis bands, in the visible and near-infrared, are much weaker than the aforementioned bands but, because they absorb near the peak of the solar spectrum, the energy deposition into the atmosphere is significant. Furthermore, this deposition takes place in the lower atmosphere and so is particularly relevant for climate. The absorption of solar near-infrared by carbon dioxide and water vapour is smaller but makes an important contribution to the heat budget of the lower atmosphere.

The spectral composition of solar irradiance is thus important in determining at what altitudes it is absorbed and produces local heating.

Figure 25 presents a vertical profile of diurnally averaged solar heating rates for equatorial equinox conditions, showing the contribution of each of the UV/vis absorption bands mentioned above. This vertical structure in the absorption of solar radiation is crucial in determining the profile of atmospheric temperatures and plays an important role in atmospheric chemistry and thus composition. Not shown in Figure 25 is the contribution of near-infrared solar radiation to heating the troposphere, due to its absorption by water vapour, oxygen and minor constituents.

\subsection{Variability of solar spectral irradiance and heating rates}

From the above discussion it is clear that the response of the middle atmosphere to solar variability will depend critically on the spectral composition of the changes in solar irradiance. The UARS satellites provided measurements of the solar UV spectrum during the 1990s. More recently the spectral range has been extended into the visible and near infrared through the instruments on ERS-2, 1994-2003 (http://www.iup.uni-bremen.de/gome/), EnviSat, 2002-present (http: //www.iup.uni-bremen.de/sciamachy/) and SORCE, 2003-present (http://lasp.colorado. edu/sorce/). Otherwise estimates are based on semi-empirical models. Figure 26 presents variations in spectral irradiance deduced for an 11-year solar cycle corresponding to a peak-to-peak

Living Reviews in Solar Physics

http://www. livingreviews.org/lrsp-2007-2 


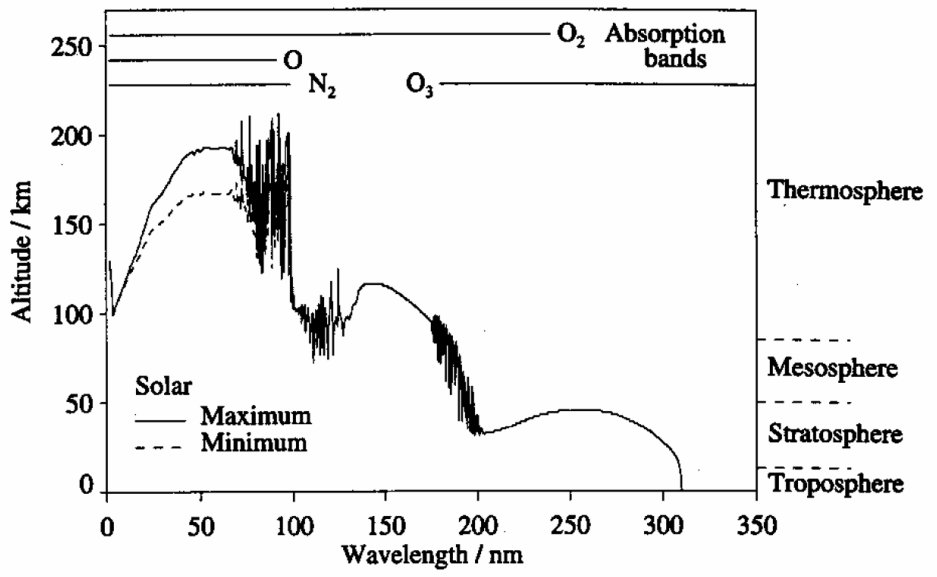

Figure 24: Wavelength dependence of the altitude of one optical depth for absorption of solar radiation with an overhead Sun. After Andrews (2000).

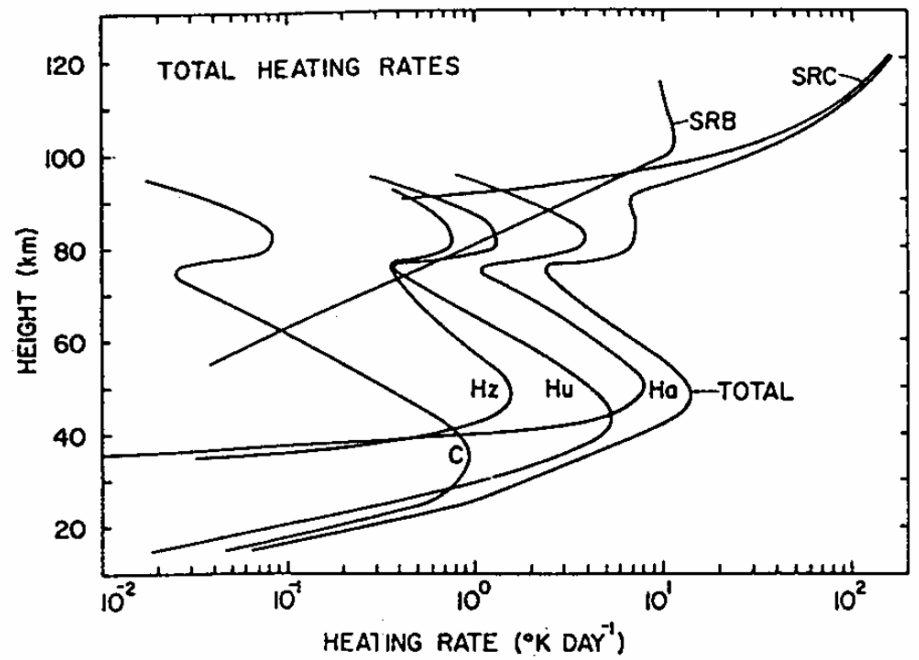

Figure 25: Diurnal average solar heating rate ( $K d-1$, log scale) as a function of altitude for equinoctial conditions at the equator showing contributions by the Schumann-Runge continuum and bands (SRC and SRB), the Herzberg continuum (Hz) and the Hartley (Ha), Huggins (Hu) and Chappuis (Ch) bands. After Strobel (1978). 

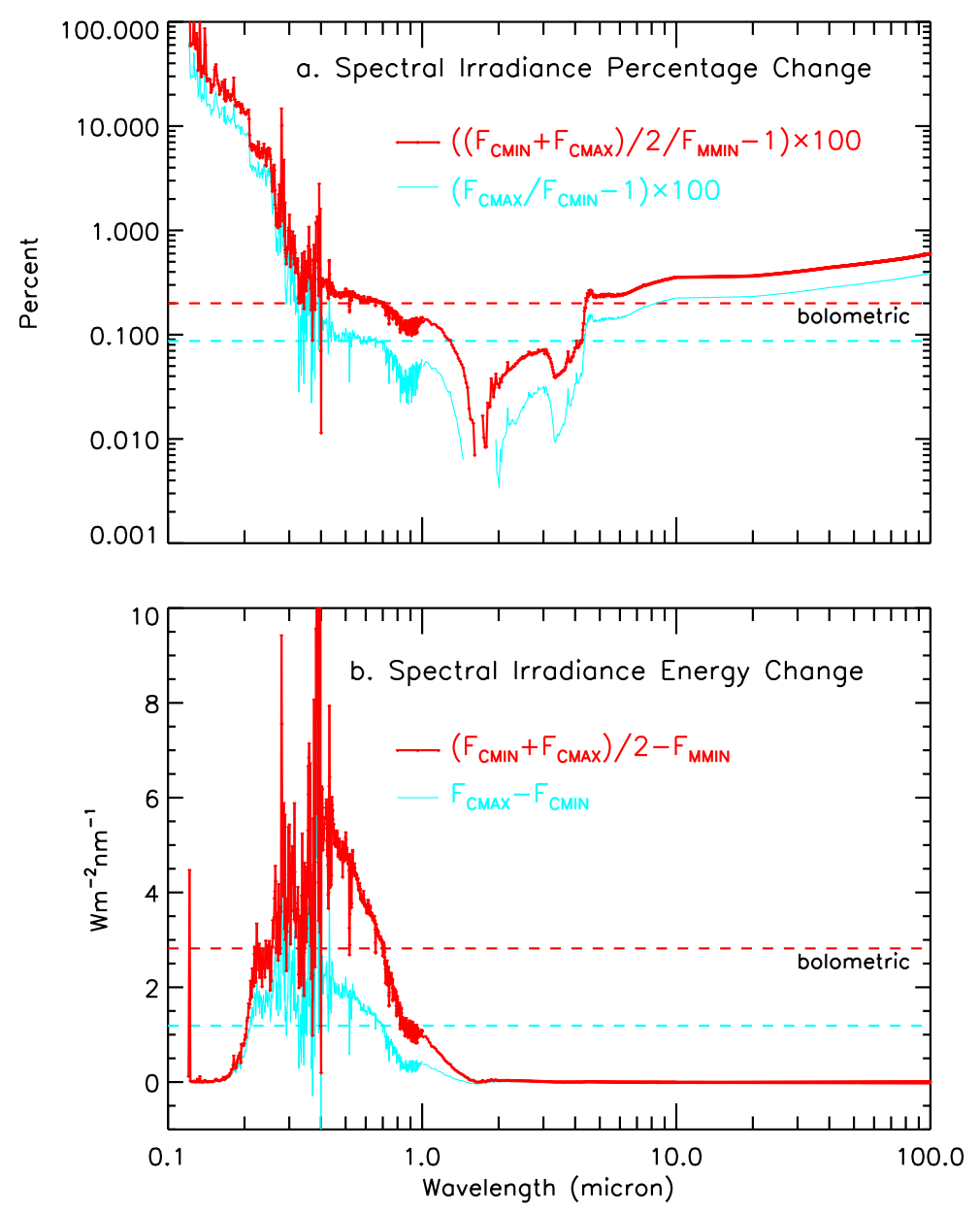

Figure 26: Estimated changes in solar spectral irradiance from cycle minimum to maximum (blue) and from the Maunder Minimum to present day mean of the solar cycle (red) expressed as percentage changes (above) and energy changes (below). From Lean (2000). 
TSI variation of $1.2 \mathrm{Wm}^{-2}$ (similar to solar cycles 21 and 22). This figure also shows the difference in spectral irradiance between a current cycle mean and the Maunder Minimum. Although, as discussed above, the amplitude of the TSI variation over this period is uncertain, the relative spectral variations are probably more reliable.

It is apparent that much larger fractional changes take place at shorter wavelengths than the $\sim 0.1 \%$ variation in TSI, shown in Figure 22, which represents the changes in the visible portion of the spectrum. Thus the direct impact of solar irradiance variability is larger in the middle and upper atmosphere than it is at lower altitudes.

Figure 27 (upper panels) shows vertical profiles of the solar fluxes and heating rates for solar minimum conditions in the ultraviolet, visible and near infrared spectral regions. The magnitude of the UV flux is much smaller than in the other two regions but its absorption by ozone, coupled with low atmospheric density, causes the largest heating rates in the middle atmosphere. The weaker absorption of visible radiation in the lower stratosphere and of near infrared radiation in the troposphere give much smaller heating rates. The right-hand panel reproduces the data of the centre panel with a linear pressure scale for the ordinate (emphasising the troposphere) and expanded abscissa scale.

Also shown in Figure 27 (lower panels) are the differences in fluxes and heating rates between 11-year solar cycle minimum and maximum conditions, based on the spectral differences shown in Figure 26. At the top of the atmosphere the increases in incoming radiation in the visible and UV regions are of similar magnitude but the stronger absorption of UV produces much greater heating. The spectral changes prescribed for these calculations were such that near-infrared radiation was actually weaker at solar maximum so decreases in heating rate are shown. This is contentious but the changes are anyway very small being about one part in ten thousand.

\subsection{Stratospheric photochemistry}

If the solar spectral irradiance varies then, without a change composition, the spectral heating rate varies in proportion to this change. If, however - as is actually the case, the atmospheric composition also responds to solar variability then this will affect solar fluxes and heating rates in a non-linear fashion. Particularly important in this context is stratospheric ozone.

In the stratosphere the main chemical reactions determining the concentration of ozone are:

$$
\begin{array}{llr}
\mathrm{O}_{2}+h \nu & \rightarrow & \mathrm{O}+\mathrm{O} \\
\mathrm{O}+\mathrm{O}_{2}+\mathrm{M} & \rightarrow & \mathrm{O}_{3}+\mathrm{M} \\
\mathrm{O}_{3}+h \nu & \rightarrow & \mathrm{O}_{2}+\mathrm{O} \\
\mathrm{O}+\mathrm{O}_{3} & \rightarrow & 2 \mathrm{O}_{2} \\
\mathrm{O}_{3}+\mathrm{X} & \rightarrow & \mathrm{XO}+\mathrm{O}_{2} \\
\mathrm{XO}+\mathrm{O} & \rightarrow & \mathrm{X}+\mathrm{O}_{2}
\end{array}
$$

The first of these reactions represents the photodissociation of oxygen at wavelengths less than $242 \mathrm{~nm}$. This process is the key step in ozone formation because the oxygen atoms produced react with oxygen molecules to produce ozone molecules, as depicted in the second reaction (the M represents any other air molecule whose presence is necessary to simultaneously conserve momentum and kinetic energy in the combination reaction). Because the short wavelength ultraviolet radiation gets used up as it passes through the atmosphere, concentrations of atomic oxygen increase with height. This would tend to produce a similar profile for ozone but the effect is counterbalanced by the need for a 3-body collision (reaction 2) which is more likely at higher pressures (lower altitudes). Thus a peak in ozone production occurs at around $50 \mathrm{~km}$. The third reaction is the photodissociation of ozone, mainly by radiation in the Hartley band $(\lambda<310 \mathrm{~nm})$, into one atom and one molecule of oxygen. This does not represent the fundamental destruction of the ozone because the oxygen atom produced can quickly recombine with an oxygen molecule. The fourth 

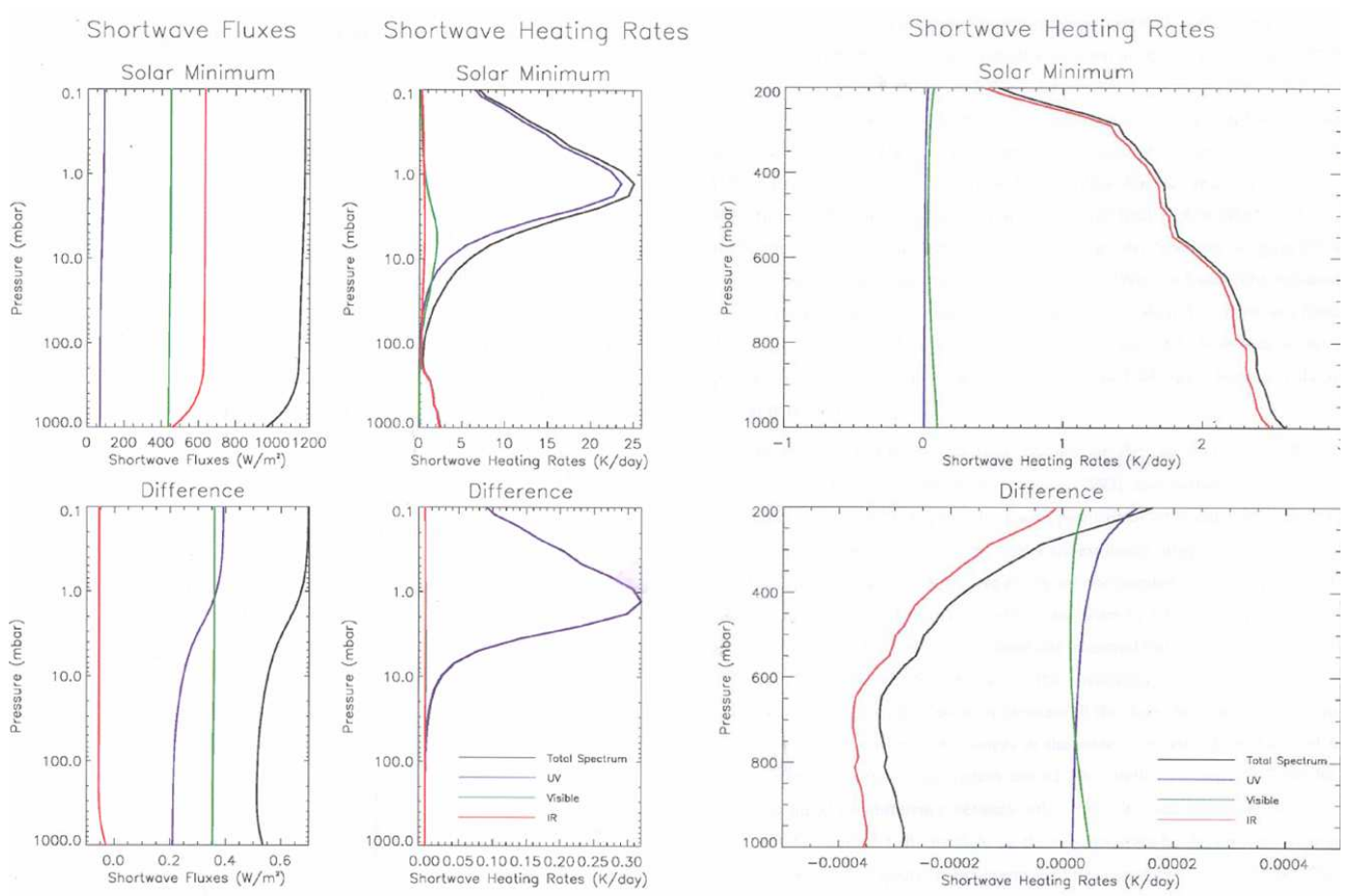

Figure 27: Solar fluxes (left) and heating rates (middle and right). Top: solar minimum values; bottom: difference between solar min and max. The spectrum is divided into UV (220-320 nm), visible (320-690 nm) and near infrared (690-1000 nm) bands. The right hand column has a linear pressure scale for the ordinate so emphasising the troposphere. From Larkin (2000). 
reaction represents the destruction of ozone by combination with an oxygen atom. The fifth and sixth reactions represent the destruction by any catalyst $\mathrm{X}$, which may include $\mathrm{OH}, \mathrm{NO}$ and $\mathrm{Cl}$. The various destruction paths are important at different altitudes but the combined effect is an ozone concentration profile which peaks near $25 \mathrm{~km}$ in equatorial regions.

Because photodissociation is an essential component of ozone formation most ozone is produced at low latitudes in the upper stratosphere. Observations show, however, that it is also present in considerable quantities in the mid- and high latitude stratosphere due to transport by the mean meridional circulation. The resulting distribution of mixing ratio is shown in Figure 28 (upper panel). The atmospheric circulations tend to move the ozone away from its source region towards the winter pole and downwards. Indeed, the quantity of ozone above unit area of the Earth's surface (referred to as the ozone column amount) is usually greater at mid-latitudes than the equator, see Figure 28 (lower panel). In the lower stratosphere its photochemical lifetime is much longer, because of the reduced penetration of the radiation which destroys it, and its distribution is determined by transport, rather than photochemical, processes. In winter high latitudes photochemical destruction essentially ceases and the ozone accumulates until the spring.

The minimum in ozone column which occurs near the south pole in spring (October) has deepened considerably during the past thirty years into what has become known as the "ozone hole". Observations and theoretical studies have shown that the depletion occurs mainly in the lower stratosphere and is due to catalytic destruction of ozone by chlorine radicals activated on the surface of polar stratospheric cloud particles during the polar night. This chlorine is a product of the breakdown of anthropogenic chlorofluorocarbons (CFCs) which are now banned under international agreement. Because of the long atmospheric lifetime of CFCs, and exacerbation of the situation by colder stratospheric temperatures associated with greater $\mathrm{CO}_{2}$ concentrations, it may be several decades before the winter polar stratosphere recovers to its unperturbed state.

\subsection{Solar cycle signal in stratospheric ozone}

It is clear from the discussion of Section 5.3 that ozone is produced by short wavelength solar ultraviolet radiation and destroyed by radiation at somewhat longer wavelengths. Because the amplitude of solar cycle variability is greater in the far ultraviolet (see Section 5.2) ozone production is more strongly modulated by solar activity than its destruction and this leads to a higher net production of stratospheric ozone during periods of higher solar activity. Observational records (Figure 29a) suggest a peak in ozone response of about $2 \%$ over the solar cycle in the upper stratosphere, with a secondary maximum in the lower stratosphere, although the restricted length of the data series means that these results are not yet statistically robust. Ozone column (Figure 29b) shows $0.5-4 \%$ higher values in ozone columns at 11-year cycle maximum relative to minimum.

Solar activity is manifest not only in variations of the Sun's emission of electromagnetic radiation but also in a range of other parameters. One of these is the occurrence and severity of coronal mass ejections which result in the emission of energetic particles, some of which reach the Earth. Precipitating electrons and solar protons follow the Earth's magnetic field lines and so have greatest initial impact at high latitudes. They affect the nitrogen oxide budget of the middle atmosphere through the ionization and dissociation of nitrogen and oxygen molecules. NO catalytically destroys ozone, as discussed in Section 5.3, so that solar energetic particle events are associated with reductions in atmospheric ozone (see Figure 30). The highest energy particles penetrate well into the stratosphere and ozone depletion regions may propagate downwards and equatorwards over the period of a few weeks (Jackman et al., 2006). It is interesting to note that the effect of energetic particle events on ozone is in the opposite sense to that of enhanced ultraviolet irradiance. Particle events tend to occur more often when the Sun is in a declining phase of the solar cycle so the combined effect on ozone may be complex in its geographical, altitudinal and temporal distribution and may confuse regression analyses (such as shown in Figure 29) which use 

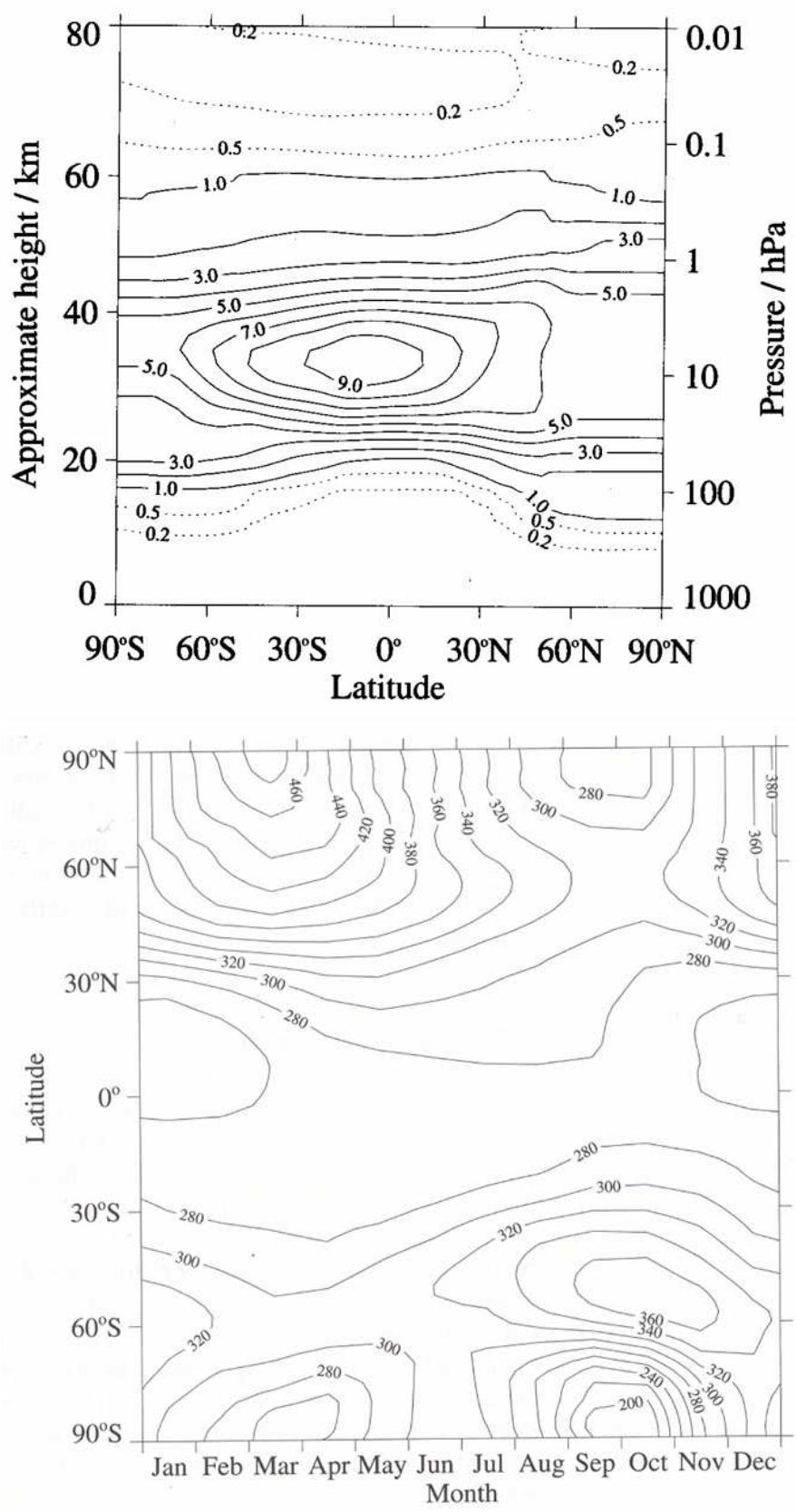

Figure 28: Above: Zonal mean concentration of ozone (ppmv) in January. Below: The annual cycle in the latitudinal distribution of zonal mean ozone column amounts. The units are $10^{-3} \mathrm{~atm} \mathrm{~cm}$ $\left(1 \mathrm{~atm} \mathrm{~cm}=2.69 \times 10^{19}\right.$ molecules $\left.\mathrm{cm}^{-2}\right)$. From Andrews (2000). 
a) annual solar (\%/100 F10.7)

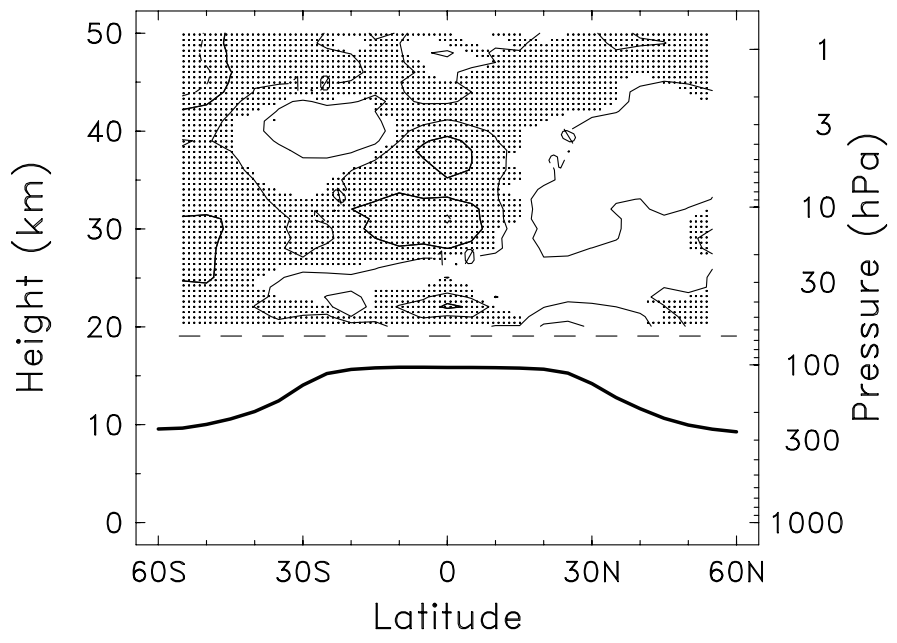

b)

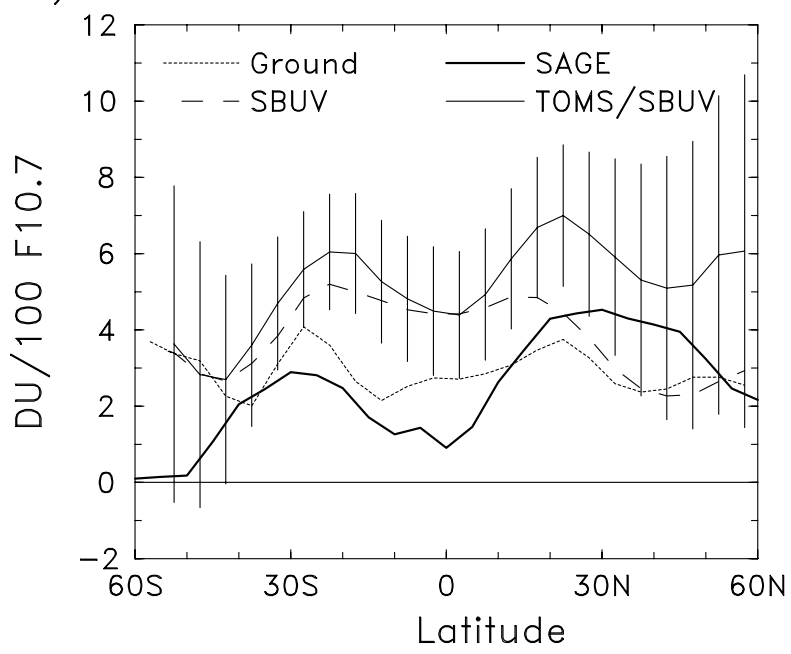

Figure 29: (a) Meridional cross section of solar cycle signal in ozone concentration from multiple regression of SAGE I and II data (\% per 100 units of $F_{10.7}$ radio flux, a typical solar cycle amplitude is 130 of these units). Shading denotes that the fit is not statistically significant. (b) Latitudinal profile of the solar cycle variations in column ozone $(a t m \mathrm{~cm})$ derived from vertically integrated SAGE I and II data (over 20-50 km), and three column ozone data sets (ground-based, SBUV, and merged TOMS/SBUV data). Error bars on the TOMS/SBUV curve denote 2*sigma uncertainty in the fit. From Randel and Wu (2007). 
only radiative indicators of solar activity, especially at higher latitudes.

\subsection{Impact of the stratosphere on solar radiative forcing of climate}

In Section 3.2 it was noted that the most accurate estimates of climate radiative forcing are those which take into account the effect of changes in the stratosphere on the radiative flux at the tropopause. Thus a better estimate of radiative forcing to solar irradiance changes should incorporate the effects of the influence of variations in UV on stratospheric temperature and composition (as first noted by Haigh, 1994). Figure 31 (above) shows solar irradiance at winter mid-latitudes as a function of wavelength and altitude calculated using a 2D atmospheric model with fairly complete representations of photochemical and radiative processes. At the top of the atmosphere most energy is at visible wavelengths and this is transmitted almost unaffected through to the surface. At wavelengths shorter than about $300 \mathrm{~nm}$, however, most radiation is bsorbed by the time it reaches an altitude of around $40 \mathrm{~km}$. There is also some absorption at longer visible wavelengths.

Table 3: A summary of published estimates of solar radiative forcing. 1st column: reference; 2nd: nominal solar variability; 3rd and 4th: solar UV radiative forcing at the top of atmosphere and at the tropopause; 5th solar-induced ozone change; 6th, 7th and 8th: impact of ozone change on shortwave and longwave components of radiative forcing and the net effect; 9th: percentage amplification of solar forcing due to change in ozone.

\begin{tabular}{|c|c|c|c|c|c|c|c|c|}
\hline Author & $\begin{array}{l}\text { Solar } \\
\text { change }\end{array}$ & $\begin{array}{l}\Delta S \mathbf{R F} \\
\text { (toa) }\end{array}$ & $\begin{array}{l}\Delta S \mathbf{R F} \\
\text { (tpse) }\end{array}$ & $\Delta \mathrm{O}_{3}$ & $\begin{array}{l}\mathrm{O}_{3} \mathrm{SW} \\
\text { effect }\end{array}$ & $\begin{array}{l}\mathrm{O}_{3} \mathrm{LW} \\
\text { effect }\end{array}$ & $\begin{array}{l}\text { Net } \mathrm{O}_{3} \\
\text { effect }\end{array}$ & $\begin{array}{l}\text { Amplifi- } \\
\text { cation } \\
(\%)\end{array}$ \\
\hline $\begin{array}{l}\text { Haigh } \\
\text { (1994) }\end{array}$ & $\begin{array}{l}\text { 11-year } \\
\text { amp }\end{array}$ & 0.13 & 0.11 & $\begin{array}{l}\text { +ve peak } \\
\text { near } \\
40 \mathrm{~km}\end{array}$ & -0.03 & +0.02 & -0.01 & -9 \\
\hline $\begin{array}{l}\text { Hansen } \\
\text { et al. } \\
(1997)\end{array}$ & $\begin{array}{l}\text { 11-year } \\
\text { amp }\end{array}$ & 0.13 & 0.11 & $\begin{array}{l}+ \text { +ve } 10- \\
150 \mathrm{hPa}\end{array}$ & & & +0.05 & +45 \\
\hline $\begin{array}{l}\text { Myhre } \\
\text { et al. } \\
\text { (1998) }\end{array}$ & $\begin{array}{l}\text { 11-year } \\
\text { amp }\end{array}$ & 0.13 & 0.11 & $+\mathrm{ve}$ & -0.08 & +0.06 & -0.02 & -18 \\
\hline $\begin{array}{l}\text { Wuebbles } \\
\text { et al. } \\
\text { (1998) }\end{array}$ & $\begin{array}{l}\text { c1680- } \\
\text { c1990 }\end{array}$ & $\begin{array}{l}0.49 \text { to } \\
0.70\end{array}$ & $\begin{array}{l}0.42 \text { to } \\
0.60\end{array}$ & $\begin{array}{l}\text { +ve peak } \\
\text { near } \\
40 \mathrm{~km}\end{array}$ & & & -0.13 & $\begin{array}{l}-30 \text { to } \\
-21\end{array}$ \\
\hline \multirow[t]{2}{*}{$\begin{array}{l}\text { Larkin } \\
\text { et al. } \\
(2000)\end{array}$} & $\begin{array}{l}\text { 11-year } \\
\text { amp }\end{array}$ & 0.13 & 0.11 & $\begin{array}{l}\text { +ve (as } \\
\text { Haigh, } \\
\text { 1994) }\end{array}$ & -0.06 & +0.11 & +0.05 & +45 \\
\hline & & 0.13 & 0.11 & $\begin{array}{l}\text { +ve } \\
\text { (SBUV/ } \\
\text { TOMS) }\end{array}$ & -0.03 & +0.08 & +0.05 & +45 \\
\hline $\begin{array}{l}\text { Shindell } \\
\text { et al. } \\
(2001)\end{array}$ & $\begin{array}{l}1680- \\
1780\end{array}$ & $\begin{array}{l}0.30 \text { to } \\
0.39\end{array}$ & $\begin{array}{l}0.26 \text { to } \\
0.33\end{array}$ & $\begin{array}{l}\text {-ve (up- } \\
\text { per strat) }\end{array}$ & & & +0.02 & $\begin{array}{l}+6 \text { to } \\
+8\end{array}$ \\
\hline
\end{tabular}

The lower panel of Figure 31 shows the difference in spectral irradiance between maximum 


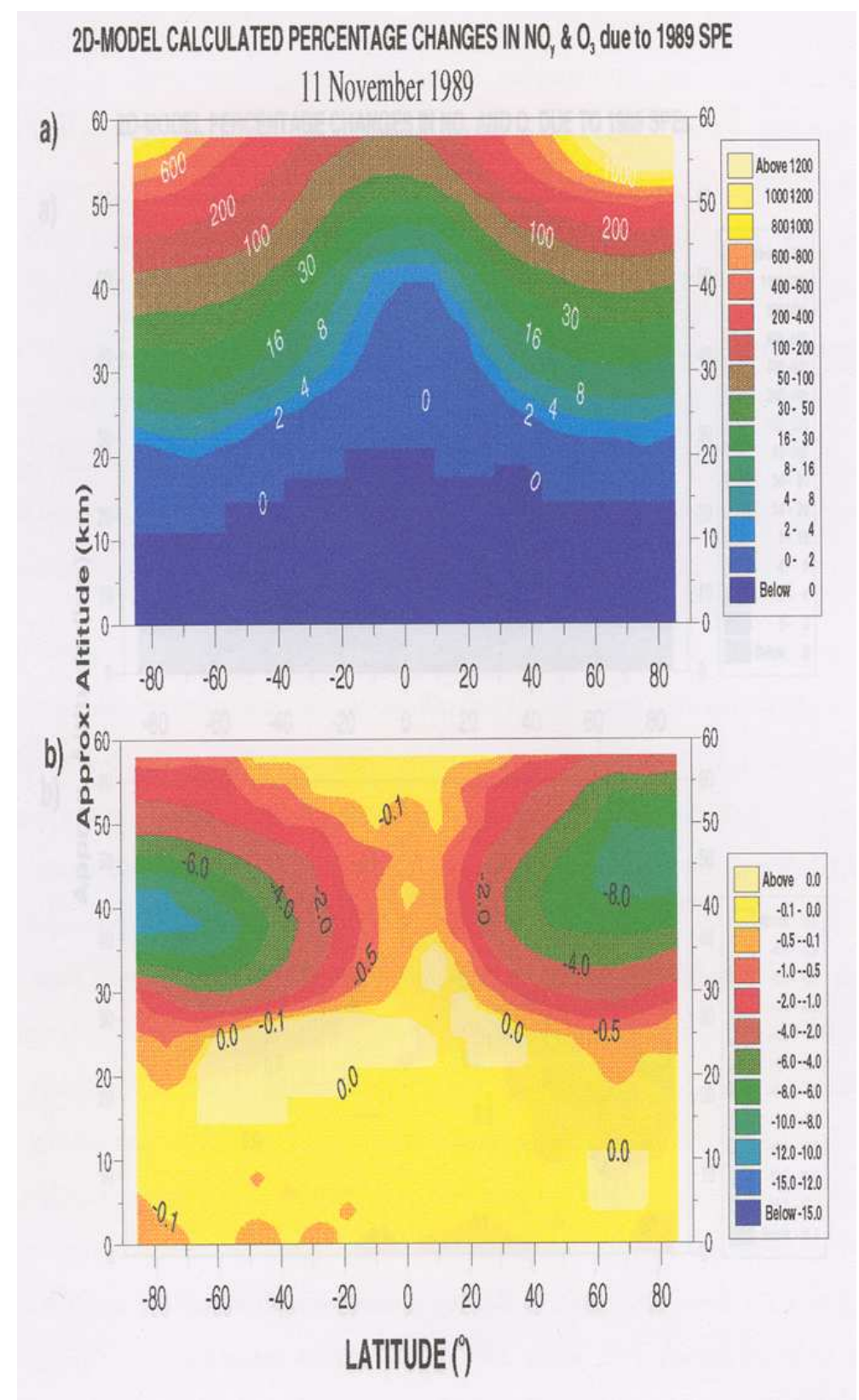

Figure 30: Latitude-height section of percentage change in $\mathrm{NO}_{y}$ (top) and $\mathrm{O}_{3}$ (bottom) calculated using a 2D model for November 1989, following the major solar proton event of October 1989. From Vlachogiannis and Haigh (1998). 

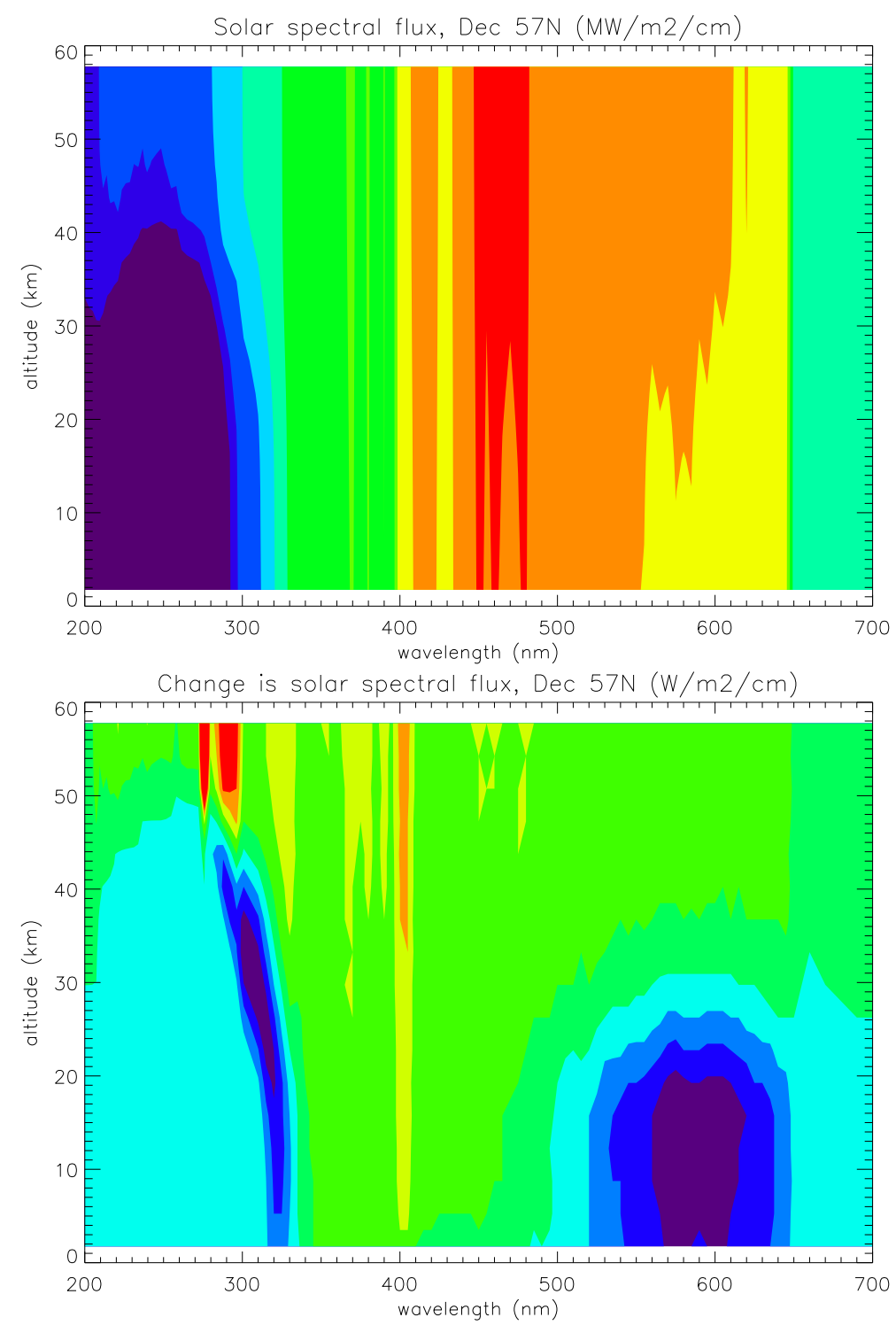

Figure 31: (Above) Solar spectral irradiance as a function of wavelength and altitude for 5ro $N$ at the winter solstice. Values range from $<10^{-4}$ (violet) to $>6$ (red) $M W^{-2} \mathrm{~cm}^{-1}$. (Below) as above but showing the difference between values at maximum and minimum periods of the 11-year solar cycle. Contour interval is $500 \mathrm{Wm}^{-2} \mathrm{~cm}^{-1}$ ranging from $<-1500$ (violet) to $>2500$ (red). After Haigh (1994). 
and minimum periods of the 11-year solar cycle. At the top of the atmosphere there is more energy at all wavelengths but this is not perpetuated throughout the depth of the atmosphere. At wavelengths $<330 \mathrm{~nm}$ and $>500 \mathrm{~nm}$ there is actually less radiation reaching the troposphere at solar maximum than solar minimum because the enhanced concentrations of stratospheric ozone are resulting in greater absorption at these wavelengths. This is a strongly non-linear effect which varies with latitude and season and thus its impact on the value of solar radiative forcing is not easy to predict. Estimates of the net effect of solar-induced ozone increases on solar radiative forcing vary widely, as can be seen in Table 3: even the sign of the ozone effect is not ascertained.

To calculate radiative forcing a knowledge of changes to the temperature of the stratosphere are also required, adding a further complication. The next section considers how well the effects of solar ultraviolet variability on the thermal structure of the middle atmosphere are understood. 


\section{Models and Mechanisms}

This section outlines potential mechanisms involved in producing some of the solar signals outlined in Sections 2.2 and 2.3. It discusses how the atmosphere may respond to changes in both total and spectrally-resolved solar irradiance through radiative and dynamical processes. It also considers how climate models can be used to test potential mechanisms and to what extent climate models are able to simulate the observed signals.

\subsection{Climate change in response to variations in total solar irradiance}

The simplest approach to simulating global average temperature changes in response to climate forcings is to use a 1-D Energy Balance Model (EBM). Figure 32 presents some of the results from one such study of the past millennium, showing the response estimated to a combination of forcing factors, along with estimates of surface temperature derived from observations. The results suggest that the gross features of the global temperature record are determined by volcanic and solar drivers until the twentieth century when human-induced factors, especially greenhouse gases, dominate.

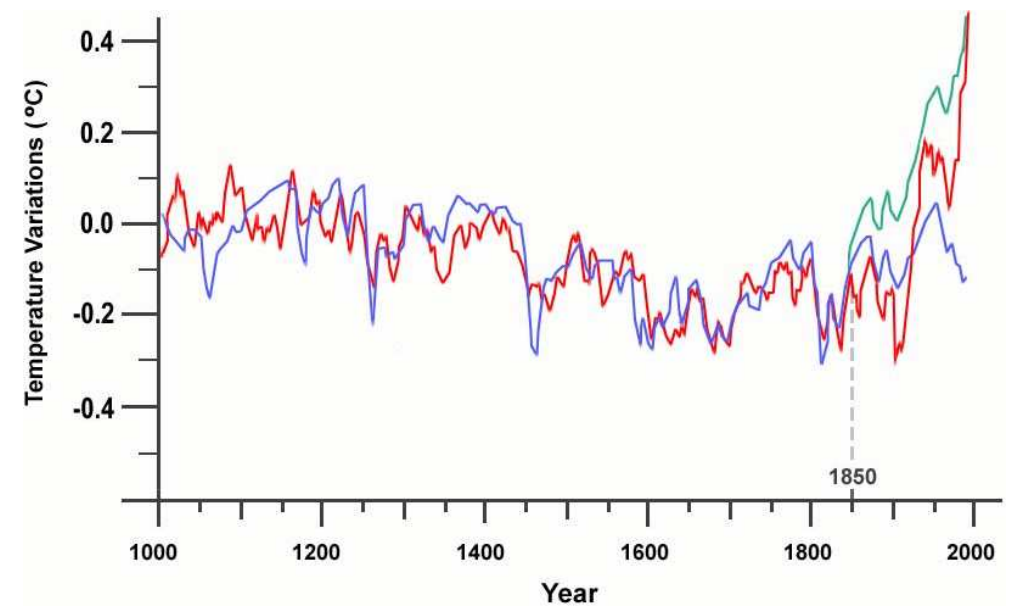

Figure 32: Global average surface temperature calculated using an EBM. Observational record (in red), model calculations with natural (solar and volcanic) forcings (in blue), difference when anthropogenic greenhouse gases are included (in green). After Crowley (2000).

Simulations with full 3-D computer models of the general circulation of the atmosphere and oceans (GCMs) have also been carried out with time-evolving natural (solar and volcanic) and anthropogenic (greenhouse gases, sulphate aerosol) forcings. The GCMs are generally able to reproduce the temporal variation of surface temperature over the past two centuries (see Figure 33). Before the mid twentieth century it is not possible to distinguish the all-forcing simulations from those only using natural forcings but since that time the models can only reproduce the observed warming if anthropogenic factors are included. This conclusion applies over each continent and the oceans as well as in the global or hemispheric means.

However, there is a large amount of natural variability and noise in both the model and observational datasets which makes detecting component causes of climate change difficult. An alternative approach is "optimal fingerprinting" in which it is assumed that the geographical patterns of response to particular factors are known, that the time-dependences of the forcing factors are known but that the amplitudes of the responses are unknown. The task is then essentially to perform

Living Reviews in Solar Physics

http://www. livingreviews.org/lrsp-2007-2 


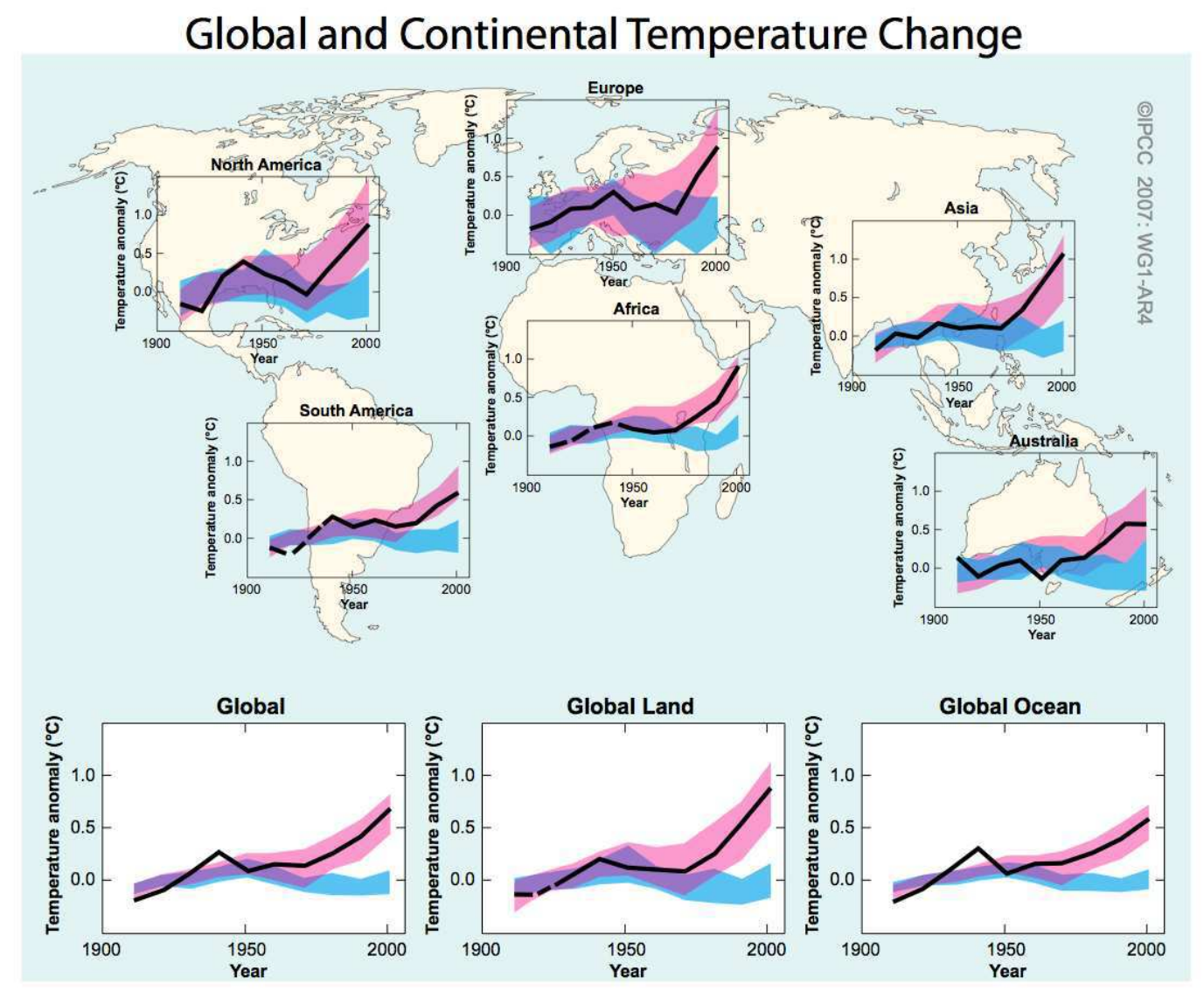

Figure 33: Annual and decadal mean surface temperature from observations (black line) and calculated using GCMs. Each panel shows calculations using all forcings (pink) and only natural forcings (blue), the spread indicates uncertainties in the estimates. From IPCC (2007). 
a multiple regression analysis on a dataset to find which weighted combination of the response patterns best matches the data, taking into account known errors/uncertainties in both the data and patterns. An example of the results of one such analysis, using a dataset of surface temperature observations on a latitude-longitude grid over the twentieth century, is shown as global averages in Figure 34a. The black curve is the observational record with the grey band representing measurement uncertainty; the red curve shows the result of using only anthropogenic forcing factors, the green only natural factors and the blue both together. A good fit is obtained when both types of forcing are included and the figure shows that increasing solar activity with declining volcanic activity were major drivers of the warming in the first half of the twentieth century and the mid-century plateau in temperatures.
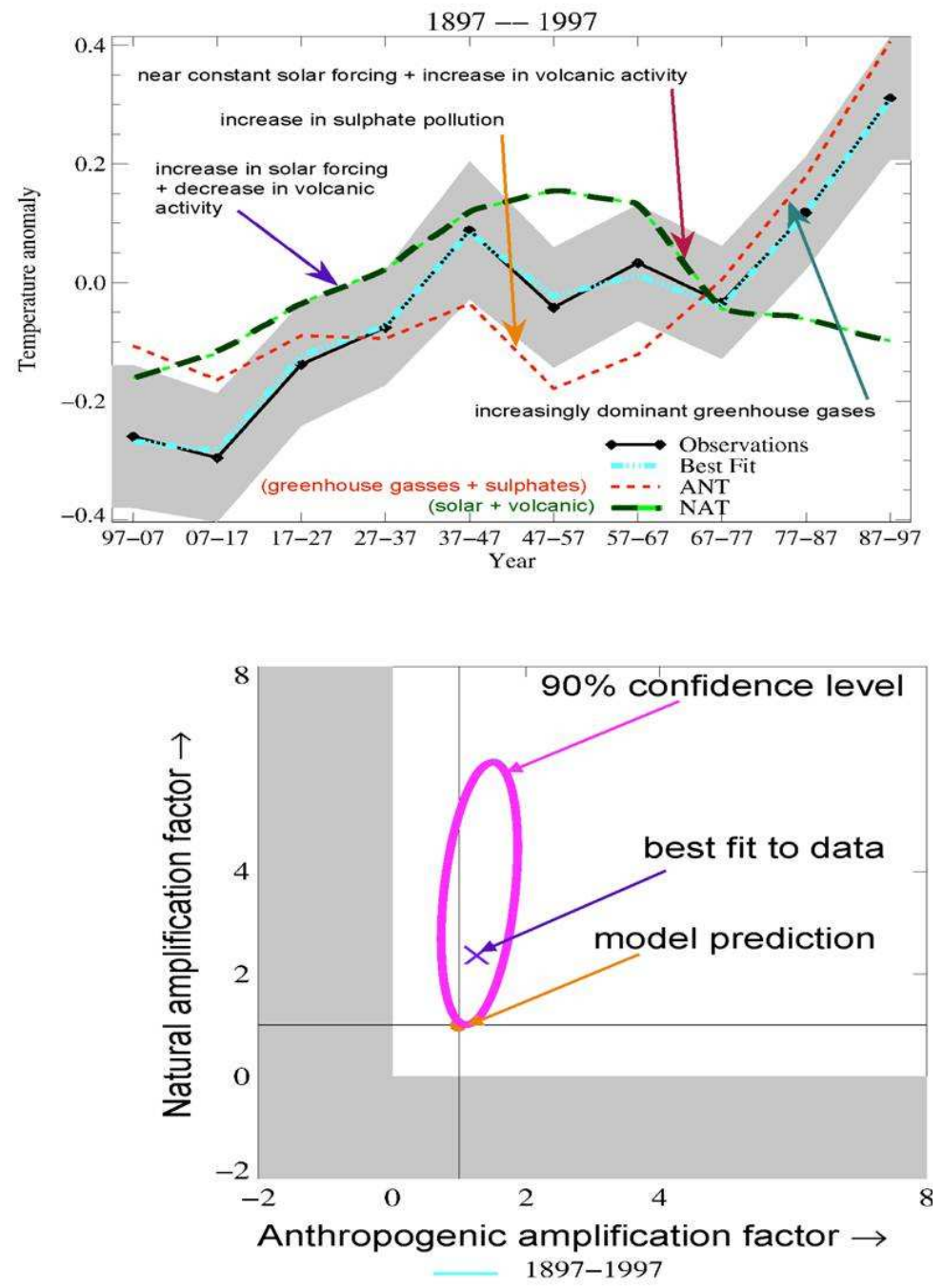

Figure 34: Optimal fingerprinting technique in which geographical patterns of surface temperature change for different forcings are fitted to the observed time series. (a) results for different forcing factors, (b) derived magnitude of natural and anthropogenic forcings relative to that found from standard model runs. From Stott et al. (2003), redrawn by M. Lockwood (personal communication). 
Figure 34b, however, shows the derived magnitudes of the forcings. Here the value 1 indicated that the derived magnitude equals that the model gives using standard radiative forcing estimates. The model appears to be underestimating the solar influence by a factor of 2 or 3 implying that some amplification factors of the solar influence are not incorporated into the model's representation.

This result, however, is sensitive to the choice of TSI reconstruction. The Stott et al. (2003) work used the Hoyt and Schatten (1998) series, which has a large secular variation, although more recent work (see the discussion in Section 4.2) is suggesting much smaller long-term variability in TSI. If the latter were used then an even larger amplification of the model's response would be required to match observations. This is an intriguing suggestion but this work needs to be reassessed using different GCMs to check that it is not an artefact of the specific one used in that study.

Nevertheless, most GCM runs which only include variations in TSI are unable to reproduce the distribution of temperature response shown in Sections 2.2 and 2.3, confirming that something is lacking in their ability to simulate the response of climate to solar activity. One recent study by Meehl et al. (2003), however, presented results from a model experiment which produced changes in SST similar to those shown in Figure 9. The authors explained these in terms of a response of the mean overturning circulations to sea surface temperature gradients enhanced between cloudy and clear regions. This intriguing possibility remains to be validated.

To explain the model underestimate it is necessary to find some factors which amplifies the effect from that derived simply by consideration of total solar irradiance as the primary driving mechanism behind the impact of solar variability on climate. Potentially one such amplification mechanism is through the effects of variations in solar UV radiation on the stratosphere.

\subsection{Model studies of the influence of varying UV in the middle atmo- sphere}

In Section 2.3 the thermal response of the stratosphere/troposphere to solar variability was shown to be largest near the tropical stratopause with lobes of warming in the sub-tropics in the lower stratosphere and bands of warming through mid-latitudes in the troposphere. One route to understanding this structure is to see if it can be reproduced in atmospheric models.

Figures 35 and 36 show zonal mean temperatures from various atmospheric GCMs in which changes to UV irradiance corresponding to the 11-year cycle variability have been imposed. In Figure 35 ozone changes have been prescribed, so that there is no feedback between the solar heating and ozone which might influence temperatures (and also circulation and winds). All these models show a similar temperature response with greatest warming near the stratopause, stretching across most latitudes. Figure 36 shows results from a model with a coupled chemistry scheme so that feedbacks between chemistry, radiation and dynamics are allowed. Here the upper stratospheric warming is more concentrated at low latitudes and there is a suggestion of a vertical structure more reminiscent of those shown in Figure 14.

A comparison between satellite observations and model predictions for the solar cycle signal in zonal mean ozone concentration is presented in Figure 37. The models include older 2D studies, which have good representations of photochemical and radiative processes but poor representation of transport, and more recent 3D coupled chemistry-climate simulations. The profiles retrieved from satellite data suggest maximum fractional change in the upper stratosphere, a minimum near $30 \mathrm{~km}$ (especially in the tropics) and possibly a second maximum below. All the models, however, predict a peak near $40 \mathrm{~km}$ with a monotonically decreasing signal below. The observational data are only available over less than two solar cycles, so there remains some doubt about the statistical robustness of the signals derived from them, and Lee and Smith (2003) have suggested that the $30 \mathrm{~km}$ minimum may be a mixing of the solar signal with one due to the quasi-biennial oscillation.

A recent intercomparison of coupled chemistry climate models (Austin, 2007) suggests that 

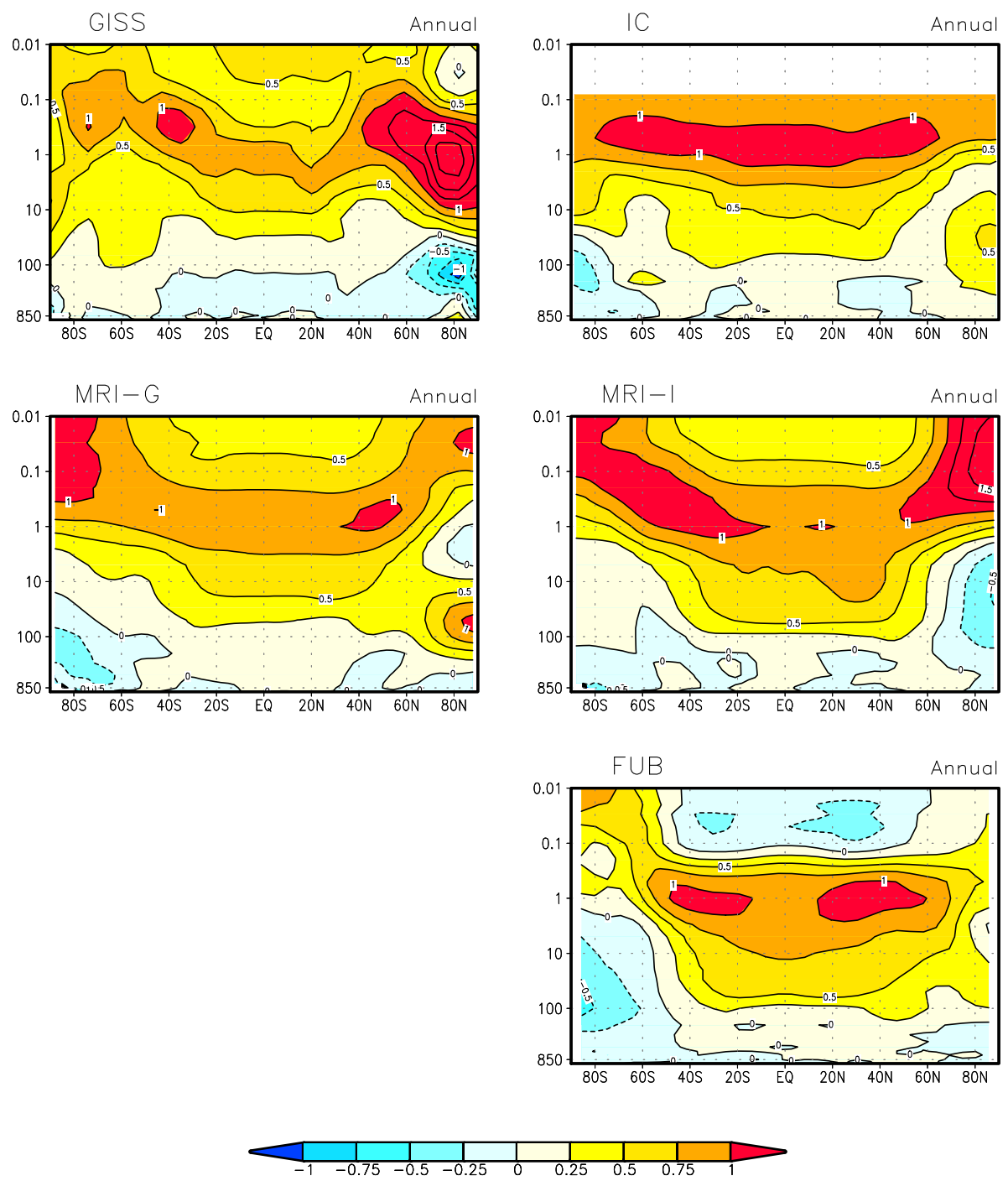

Figure 35: Results from GCM simulations of the response of middle atmospheric temperatures to the 11-year solar cycle variability in UV with different models in which the solar-induced ozone response was specified. From Matthes et al. (2003). 


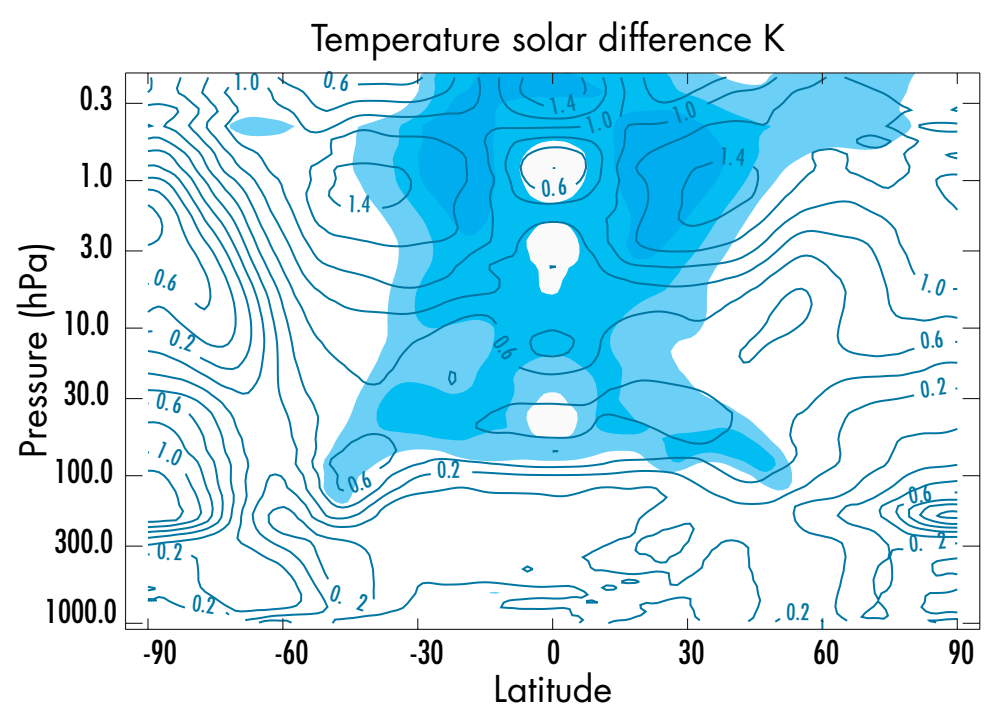

Figure 36: Results from GCM simulations of the response of middle atmospheric temperatures to the 11-year solar cycle variability in UV with a coupled chemistry scheme (shadings indicates statistical significance at the 10, 5 and 1\% levels) From Haigh et al. (2004).

this may be true for short model runs but finds that the mid-stratospheric minimum can be reproduced in simulations which include time-varying solar irradiance and prescribed sea surface temperatures. These differ from the 3D models in Figure 37 which used fixed solar max $/ \min$ scenarios with climatological SSTs. Austin (2007) argue that the transience in the simulations allows better reproduction of the mean meridional circulation of the stratosphere and thus the transport of lower stratospheric ozone. Nevertheless, another state-of-the-art coupled chemistry climate model, with very high vertical resolution (Schmidt and Brasseur, 2006) does reproduce the vertical structure with time-slice (i.e. not transient) runs. The response of stratospheric ozone to solar variability remains an active topic of research.

\subsection{Dynamical mechanisms in the middle atmosphere}

While some of the similarities between the observational analyses of solar influence and modelling studies of UV effects are intriguing they do not explain the mechanisms whereby the influence takes place. Kodera (1995) has argued that changes in the winter polar stratosphere brought about by anomalous solar heating may influence the passage of upward propagating planetary waves and thus their deposition of momentum. This argument has been developed by Kodera and Kuroda (2002) who suggest that the momentum deposition will influence the strength of the mean overturning of the stratosphere (also known as the Brewer-Dobson circulation) and thus tropical lower stratospheric temperatures (see cartoon in Figure 38).

An alternative (or additional?) perspective is provided by the work of Gray et al. (2001) who have demonstrated, using rocketsonde and satellite data that temperature anomalies in the tropical upper stratosphere (potentially related to solar activity) are correlated with subsequent temperature anomalies in the polar lower stratosphere. They also demonstrated, using a mechanistic model of the middle atmosphere that zonal wind anomalies in the sub-tropical upper stratosphere can influence the timing and amplitude of sudden stratospheric warmings - events during the polar winter in which enhanced planetary wave activity disturbs the cold polar vortex. An example is 

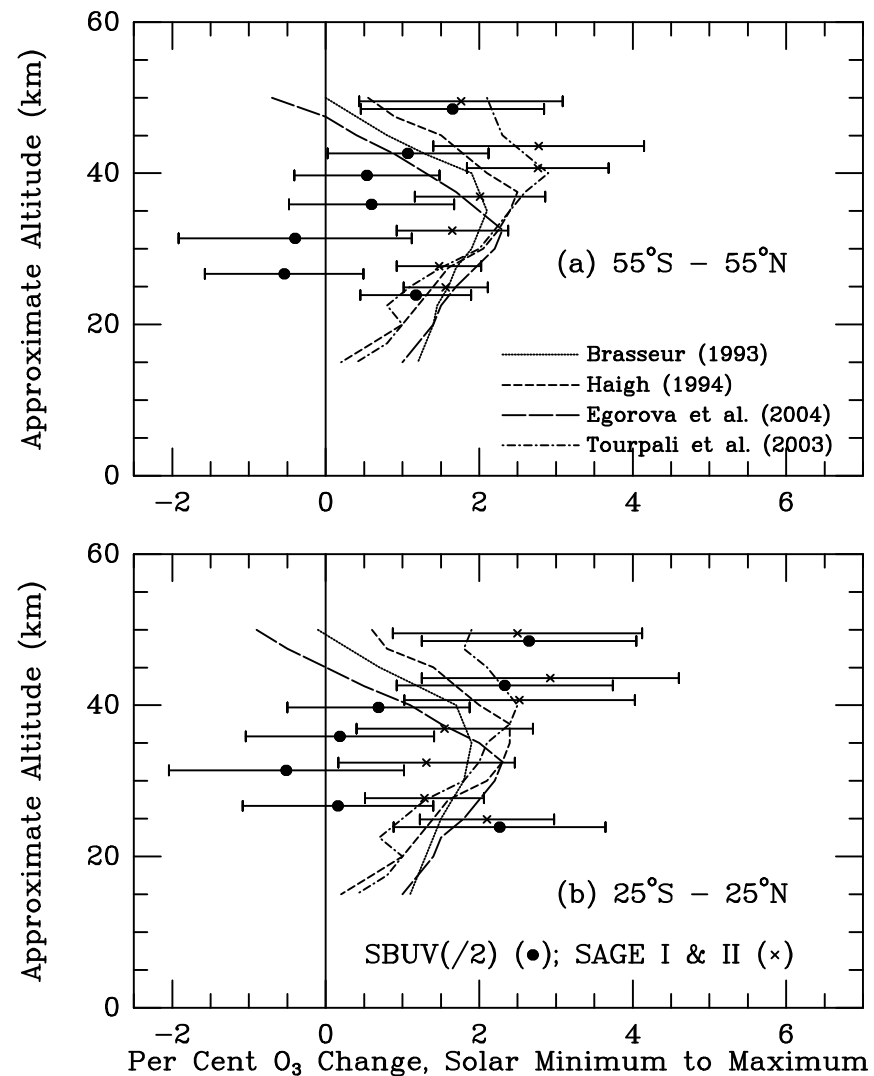

Figure 37: Estimates of percentage increase in ozone concentration from solar cycle minimum to maximum from satellite data (data points and horizontal bars) and a range of theoretical models (curves). The upper panel shows an average over most of the globe while the lower panel shows tropical data. From WMO (2007) based on data from Brasseur (1993); Haigh (1994); Egorova et al. (2004); Tourpali et al. (2003). 

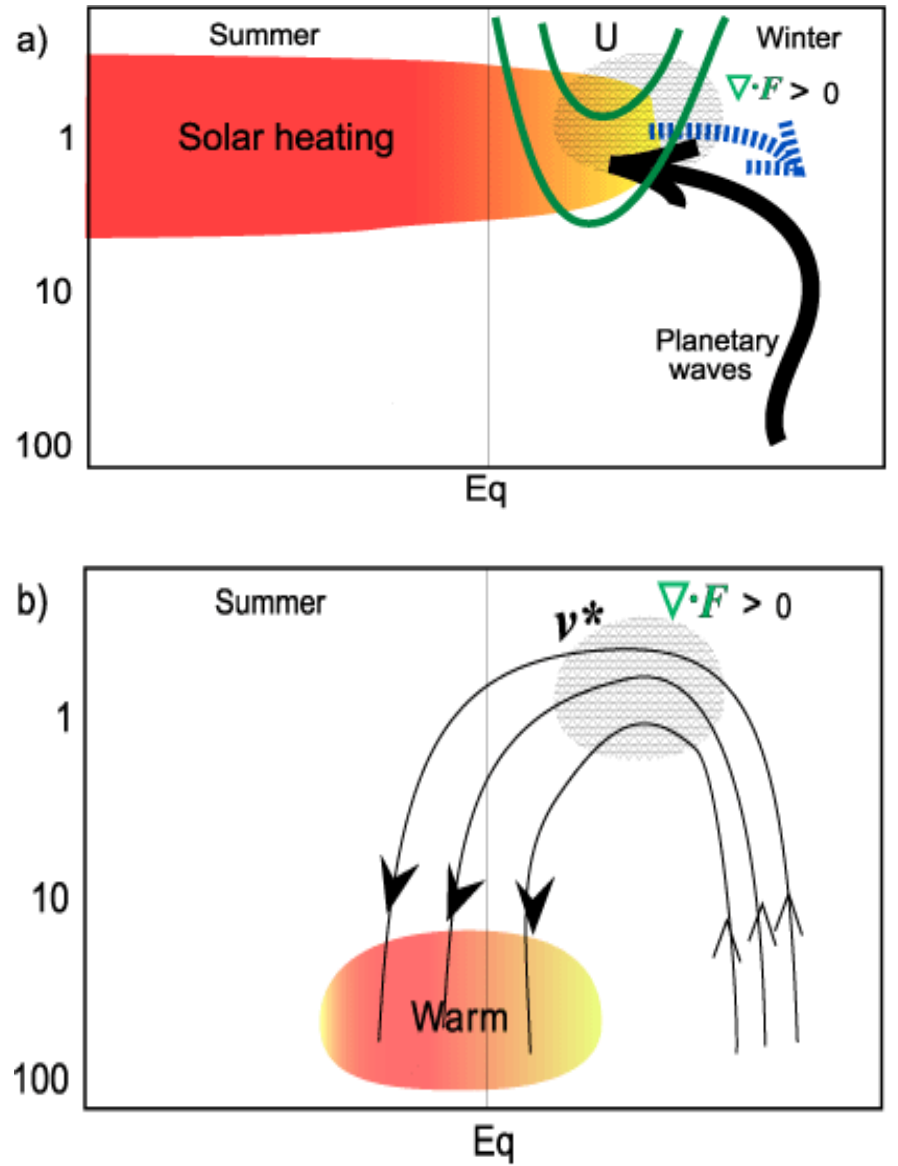

Figure 38: Illustrating the proposed mechanism whereby solar heating around the stratopause may influence the atmosphere below. (a) The solar heating anomalies change the strength of the polar stratospheric jet $(U)$; this influences the path of upward propagating planetary waves which deposit their zonal momentum on the poleward side of the jet. (b) The effect of this is to weaken the Brewer-Dobson circulation and thus to warm the tropical lower stratosphere. From (Kodera and Kuroda, 2002). 
shown in Figure 39: the unperturbed simulations (right) show random timing of warming events but when a westerly wind anomaly in forced near the stratopause in the sub-tropics of the winter hemisphere the warmings become more organised into specific time periods.
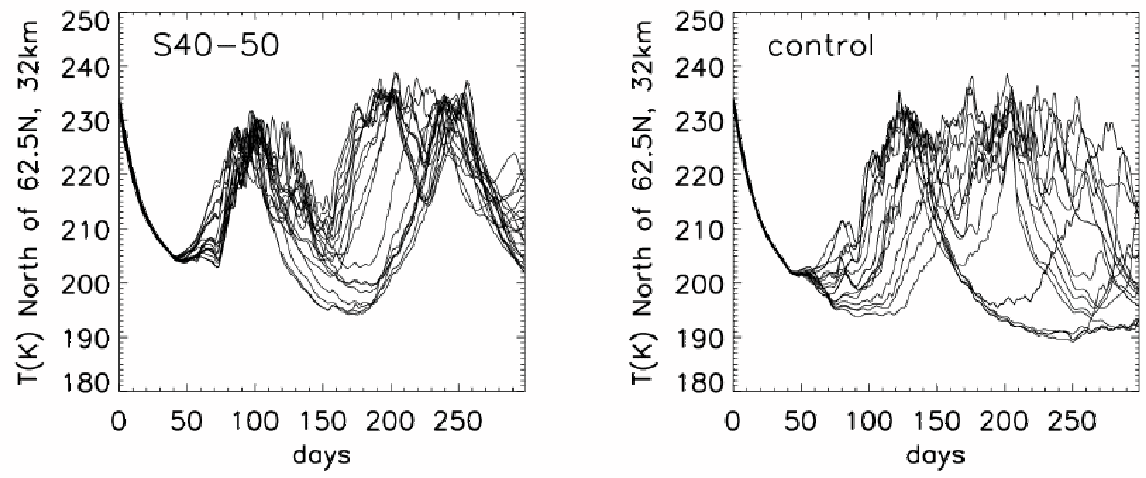

Figure 39: Temperature of the NH polar lower stratosphere evolving through the winter as calculated in a stratosphere-mesosphere mechanistic model. Each panel shows the results from twenty different simulations for each of which the initial conditions were slightly altered. Right: control situation; Left: anomalous westerly momentum applied in the winter sub-tropics near the stratopause. From Gray et al. (2004).

Gray (2003) pointed out that such a relationship between equatorial winds in the upper stratosphere and the timing of sudden stratospheric warmings could help to explain the interaction between the solar cycle and QBO influences on polar temperatures (as identified by Labitzke and van Loon, 1992, see Figure 15). Modelling evidence to support this idea has been provided by Matthes et al. (2004) who found they could reproduce the observed solar cycle/QBO polar temperature relationship if typical QBO wind profiles were imposed through the depth of the tropical stratosphere.

\subsection{Model studies of the influence of varying solar UV on the tropo- sphere}

The first GCM studies of the impact of solar UV-induced variations in stratospheric temperature and ozone on the dynamical structure of the troposphere were presented by Haigh (1996). In that paper a number of experiments were carried out using a variety of assumptions concerning ozone changes. All the experiments showed the same pattern of response in tropospheric temperatures and winds but with different magnitudes depending on the specifications of the UV and ozone changes. An example of the impact on zonal wind is shown in Figure 40 from a run in which ozone was specified based on results of a 2D model calculation. The anomaly shows that at higher solar activity the mid-latitude jets are weaker and move slightly poleward.

Figure 41 shows results from a similar model experiment for the tropospheric mean meridional circulation. The upper panel shows the mean climatological values: positive values (warm colours) indicate clockwise circulation and negative values (cold colours) anti-clockwise. The lower panel shows the anomaly associated with solar cycle variability. At solar maximum the winter Hadley cell (the major circulation in each season) is clearly weaker and broader than it is at solar minimum.

The modelled signals for zonal wind (Figure 40) and mean meridional circulation (Figure 41) are broadly similar to those deduced from observational (NCEP reanalysis) data (Figure 16 and Figure 17), although somewhat weaker in magnitude. The models used in these studies had fixed

Living Reviews in Solar Physics

http://www. livingreviews.org/lrsp-2007-2 

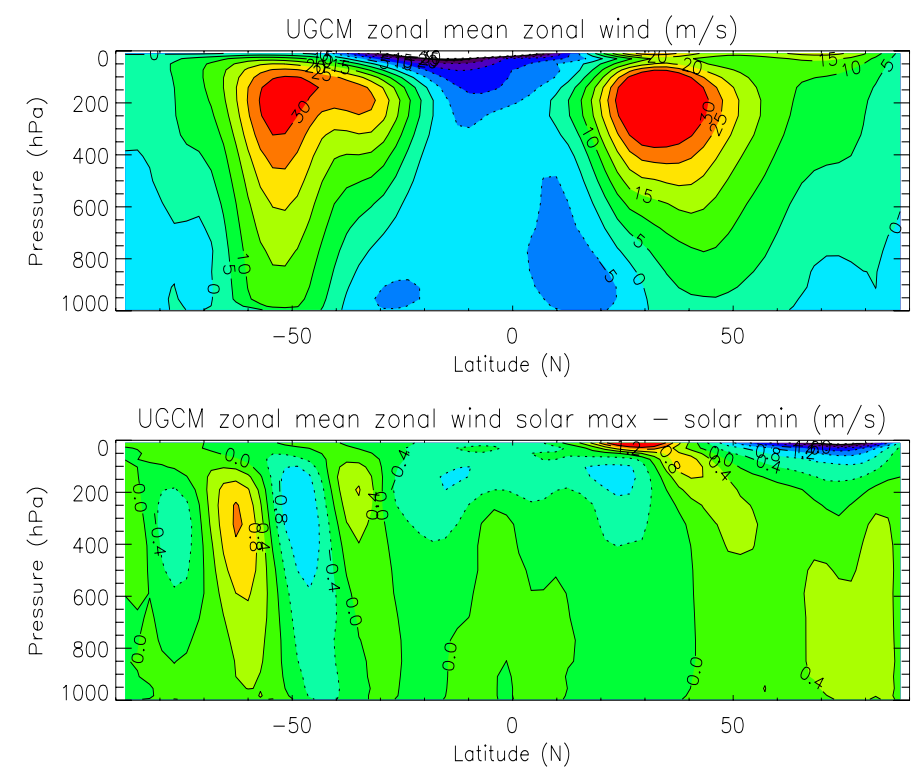

Figure 40: Above: January field of zonal mean zonal wind $\left(\mathrm{ms}^{-1}\right)$. Below: difference between fields at solar maximum and solar minimum of zonal wind calculated in a GCM. From Haigh (1996, 1999). UV changes were prescribed according to Lean (1989), ozone changes from the results of the $2 D$ model experiments of Haigh (1994).
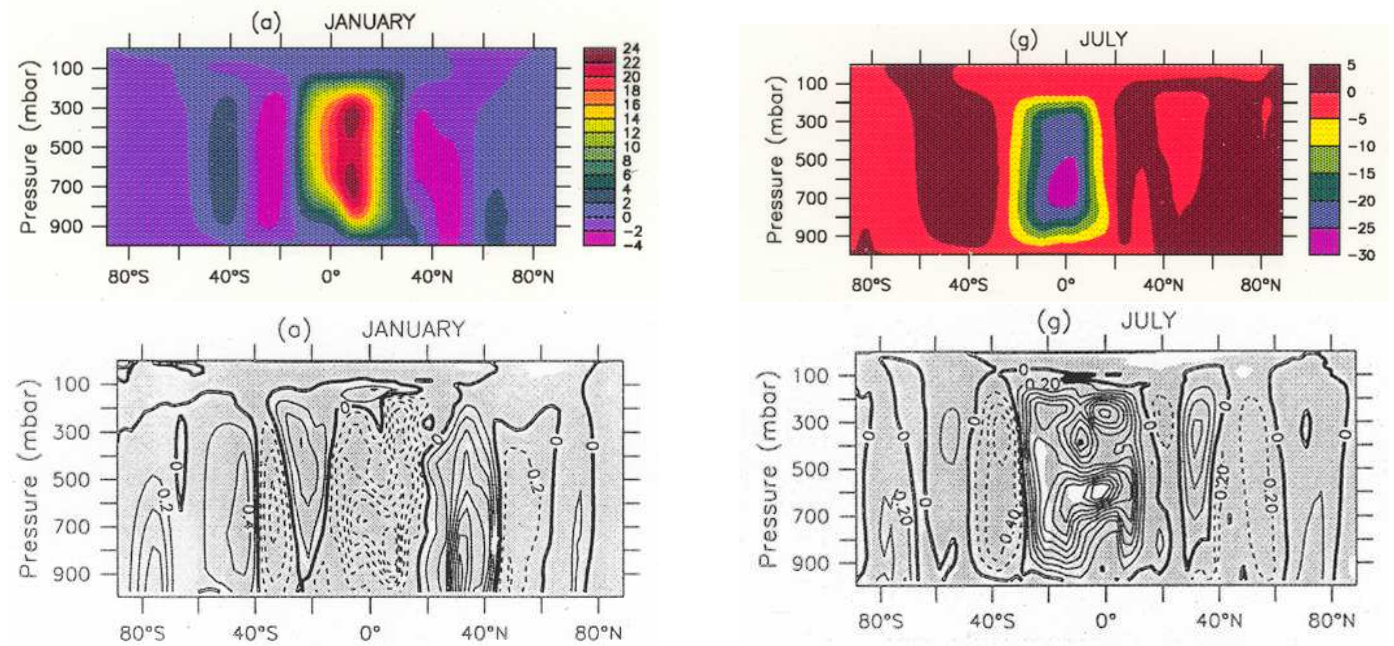

Figure 41: Mean meridional circulation. Above: mean fields for January and July. Below: difference between values at solar maximum and minimum. From Larkin (2000); similar figures in Larkin et al. (2000). 
sea surface temperatures which essentially restricted them from responses involving feedbacks between SSTs, clouds and circulation and the results suggest that at least part of the solar signal in the troposphere comes from a response to changes in the atmosphere above.

Recently an atmosphere-ocean GCM with fully coupled stratospheric chemistry has been run (despite huge computational demands) to simulate the effects of changes in solar irradiance between the Maunder Minimum and the present (Shindell et al., 2006). As in the previous studies the results show a weakened Hadley circulation when the Sun is more active, and they also suggest an impact on the hydrological cycle with greater tropical precipitation. Furthermore, they provide additional evidence that coupling with stratospheric chemistry enhances the solar signal near the surface.

\subsection{Coupling between the stratosphere and troposphere}

The Kodera and Gray studies discussed in Section 6.2 provide evidence, and mechanisms, whereby solar perturbations to the upper stratosphere may affect the lower stratosphere, in both cases enhancing any direct solar heating in this region. However, they do not explain the apparent subsequent propagation of the solar signal downwards into the troposphere.

There is some observational evidence that variations in the strength of the polar vortex in the upper stratosphere may subsequently influence surface climate. A study of polar temperature trends by Thompson et al. (2005) suggests a downward influence, and modelling experiments by Gillett and Thompson (2003) demonstrate that depletion of stratospheric ozone over the south pole can affect the troposphere after about one month. Neither of these studies is specifically concerned with a solar influence but the accumulating evidence suggests that any factor influencing the strength of the polar stratospheric jet may be able to influence surface climate, at least at high latitudes.

Similarly the apparent success of the tropospheric GCM studies (see Section 6.4) in simulating the observed response to solar variability provides intriguing evidence that changes to the stratosphere, specifically induced by variations in solar UV radiation and resulting changes in ozone, can influence the troposphere. But they do not provide a detailed understanding of the mechanisms whereby these effects take place.

Recently some effort to advance understanding of the mechanisms of stratosphere-troposphere coupling has been made through the use of simplified general circulation models (Polvani and Kushner, 2002; Kushner and Polvani, 2004; Haigh et al., 2005; Haigh and Blackburn, 2006). These models include a full representation of atmospheric dynamics but only highly-parameterised representations of radiative and cloud processes so that multiple runs can be carried out. These experiments are not intended to simulate solar (or any other specific) forcing factors but to identify and investigate possible mechanisms for stratosphere-troposphere coupling.

In the experiments of Haigh et al. (2005) two types of perturbation were applied: in the first a uniform stratospheric heating was imposed while in the second the applied heating was largest at the equator and smoothed to zero at the poles. Figure 42 shows some of the results for zonal wind. The first feature of note is that, although the perturbations were applied only in the stratosphere, a response is clearly seen in the troposphere: the uniform stratospheric perturbation causes the sub-tropical jets to weaken and move equatorward while in response to the perturbation with the latitudinal gradient the jet again weakens but moves slightly poleward. The response of the mean meridional circulation (not shown) is that the Hadley cell weakens in both cases but shrinks latitudinally in the former and expands in the latter.

The magnitudes of the perturbations applied in these experiments was much larger than might be expected from solar influences so that no direct comparison with observations is appropriate. It is clear, however, that the dynamical response to the equatorial stratospheric heating perturbation, plausibly more similar to solar forcing, is qualitatively similar to the response to solar UV found in both observations and full GCMs. Further understanding of the processes involved has been gained

Living Reviews in Solar Physics

http://www. livingreviews . org/lrsp-2007-2 
by diagnosis of the vertically integrated budget of zonal momentum. It is clear from Figure 43 that the major response is in the flux of momentum due to the zonally asymmetric eddies (i.e. synoptic-scale weather systems).
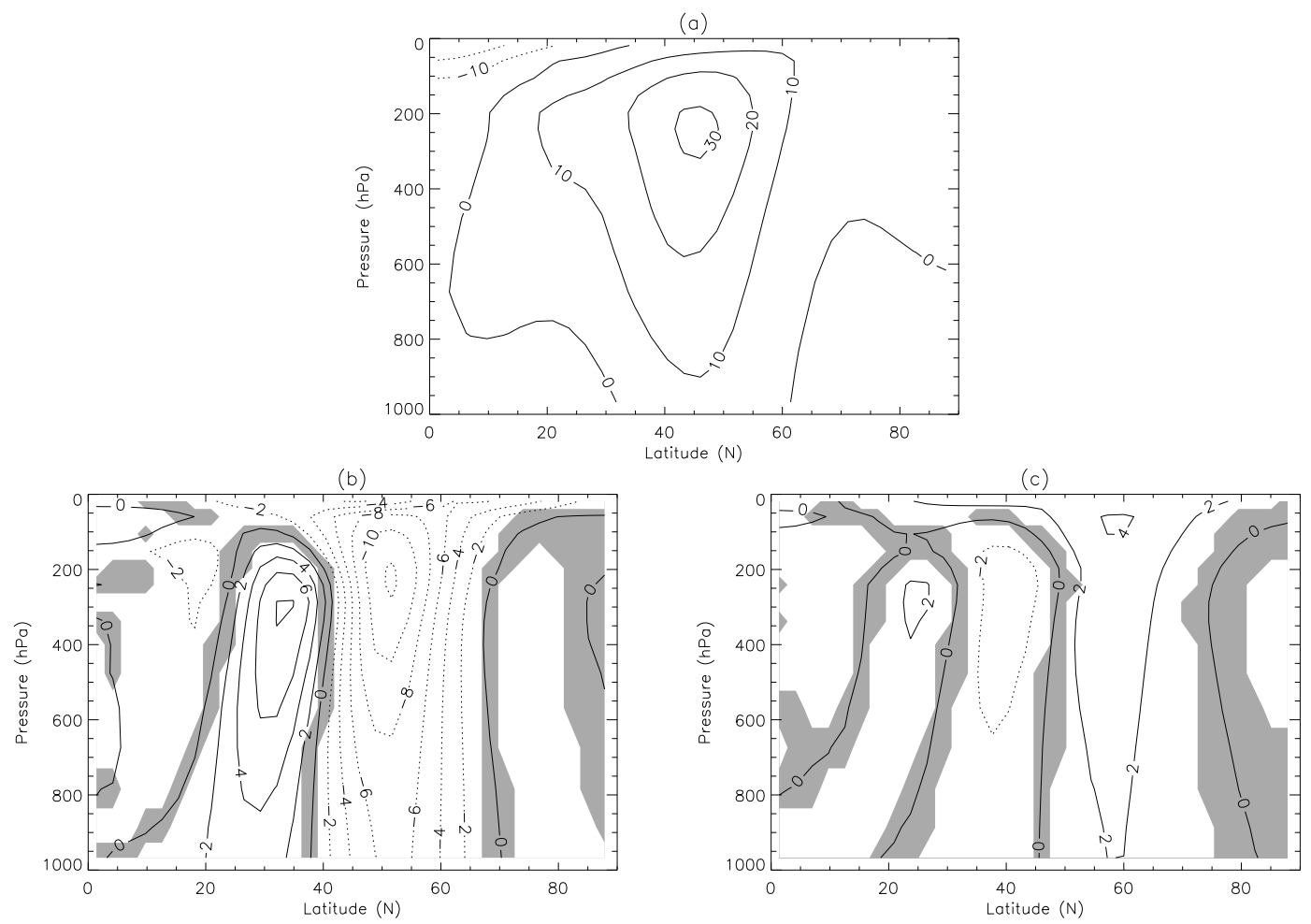

Figure 42: Results from a simplified global circulation model showing how anomalous heating of the stratosphere can influence the jets through the depth of the troposphere. (a) zonal mean zonal wind in one hemisphere as a function of latitude and pressure from the control run; (b) difference between uniform stratospheric heating case and the control; (c) difference between equatorial stratospheric heating case and the control. Shaded areas are not significant at the 5\% level. From Haigh et al. (2005).

In order to investigate the chain of causality involved in converting the stratospheric thermal forcing to a tropospheric climate signal another experiment used an ensemble of model spin-ups to analyse the time development of the response to an applied stratospheric perturbation (Haigh and Blackburn, 2006). It was found that the initial effect of the change in static stability at the tropopause is to reduce the eddy momentum flux convergence in this region. This is followed by a vertical transfer of the momentum forcing anomaly by an anomalous mean circulation to the surface, where it is partly balanced by surface stress anomalies. The unbalanced part drives the evolution of the vertically integrated zonal flow. It was concluded that solar heating of the stratosphere may produce changes in the circulation of the troposphere even without any direct forcing below the tropopause and that the impact of the stratospheric changes on wave propagation is key to the mechanisms involved.

This work is beginning to provide us with an understanding of how, through the spectral composition of solar irradiance, apparently small changes in solar irradiance may significantly impact the circulation of the lower atmosphere. 

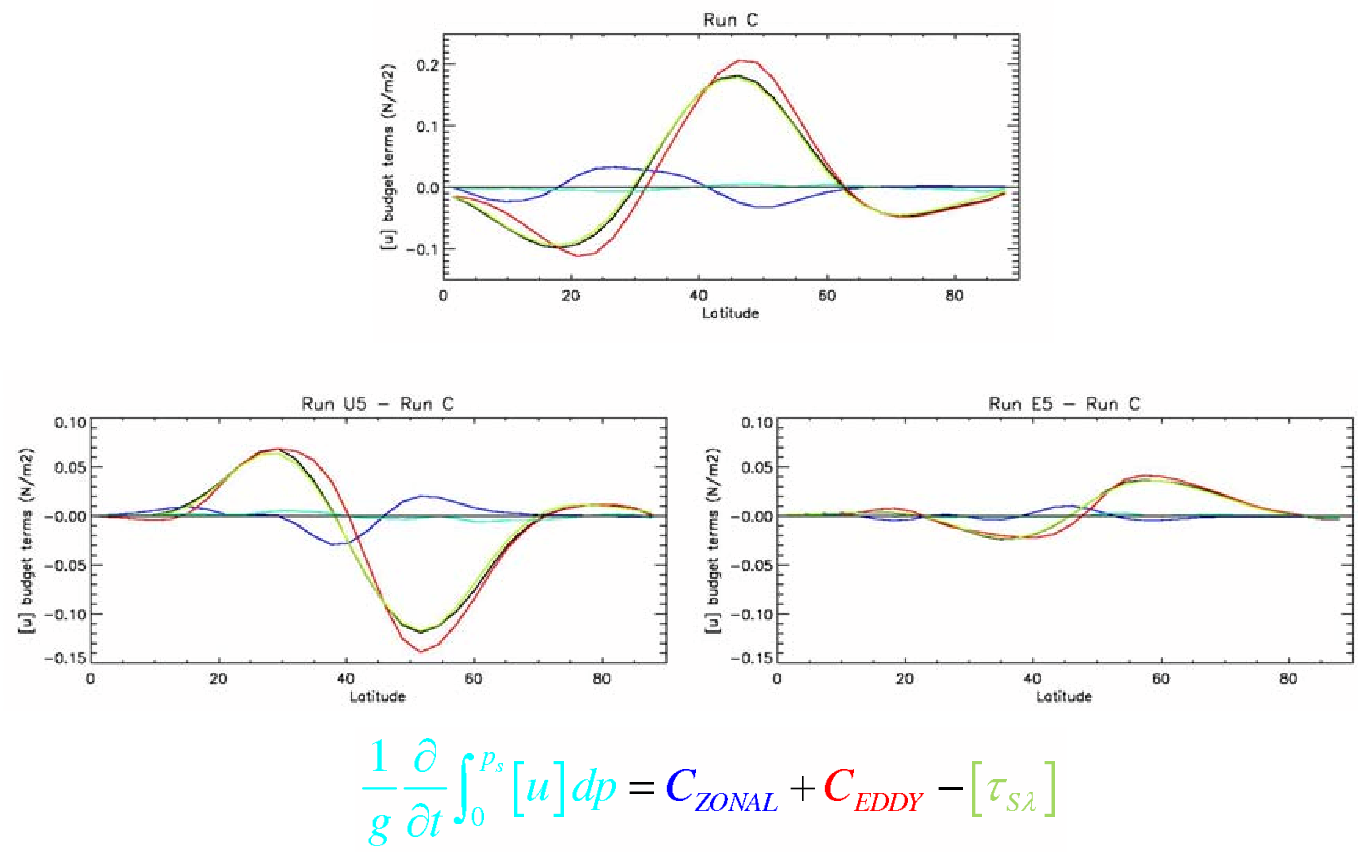

Figure 43: Vertically-integrated budget of components of the momentum equation: transport by eddies (red), transport by the zonal mean circulation (blue), dissipation by surface stress (green), sum of the eddy and zonal components (black), net rate of change of momentum (cyan), i.e. the difference between the black and green curves. Top: control run; bottom left: difference between uniform stratospheric heating case and the control; bottom right: difference between equatorial stratospheric heating case and the control. From Haigh et al. (2005). 


\section{Clouds}

Clouds play a major part in establishing the heat and radiation budgets of the atmosphere. They transport latent heat from the oceans to the atmosphere, they reflect solar radiation back to space, reducing the net incoming radiative flux, and they trap infrared radiation, acting in a similar way to greenhouse gases. Any factor influencing cloud cover thus has the potential to seriously affect climate.

In this section the role of cloud in the radiation budget, and how this impacts radiative forcing, is reviewed. There follows a brief description of how clouds are formed and how their radiative properties relate to their microphysical structure. Finally the potential for cloud cover to be modified by variations in atmospheric ionisation by cosmic rays is briefly discussed.

\subsection{Clouds and the Earth radiative budget}

Figure 18 showed that on a global average clouds increase the planetary albedo by reflecting incoming solar irradiance back to space but decrease outgoing longwave radiation by acting in a similar way to greenhouse gases. The magnitude of the reflectance depends on the optical thickness of the cloud, the water phase (liquid or ice) and the cloud particle sizes and shapes. The degree of longwave trapping depends on the transmissivity of the cloud and also its temperature: high (cold) cloud is more effective because it emits less radiation to space while trapping the (warm) radiation from below. The net effect of cloud on the radiation budget depends on whether the shortwave or longwave effect is larger and thus on the location, height and microphysical properties of the cloud.

Studies using satellite data show that there are strong regional, as well as seasonal, variations in cloud radiative effects. Figure 44 presents an analysis of radiation budget and cloud data which shows that the impact of cloud in trapping longwave radiation is greatest in the tropics. In the shortwave the largest effects on albedo are found where the optical depth of the cloud is thickest but over the bright Antarctic plateau clouds actually reduce albedo. The net impact of cloud on the radiation budget (i.e. absorbed solar radiation minus emitted thermal radiation) is negative except for small regions in the northern sub-tropics and at the south pole. Thus the global effect of cloud is to reduce the net incoming radiation, i.e. to cool the planet.

A factor which induces a change in cloud cover, drop size or altitude will introduce a radiative forcing. If, however, such a change is brought in response to another forcing factor then it should be viewed as a feedback on the initial forcing. For example, an increase in greenhouse gases might cause a surface warming, enhanced convection and an increase in cloud cover. The thick convective cloud produced would have a negative radiative forcing and thus reduce that due to the greenhouse gases alone. Such feedback effects, however, are implicitly included in the value of the climate forcing parameter $\lambda$. Thus the cloud produced by a dynamical response to other forcings can not be viewed as an additional forcing component. Only if changes to cloud properties are induced in situ by chemical or microphysical processes can they produce a radiative forcing, in the climate change sense, in their own right.

\subsection{Cloud formation}

Clouds form when the water vapour in the air condenses. Generally this occurs in air-masses which are rising, expanding and therefore cooling. The cooler air has a lower saturated vapour pressure so that the air-mass, with a given humidity, becomes saturated. Air masses rise either due to convection or through being forced to pass over some sort of barrier.

Cooling the air to saturation point is not, however, a sufficient condition for cloud to form: it is possible for the relative humidity to reach $500 \%$ without any spontaneous condensation taking 


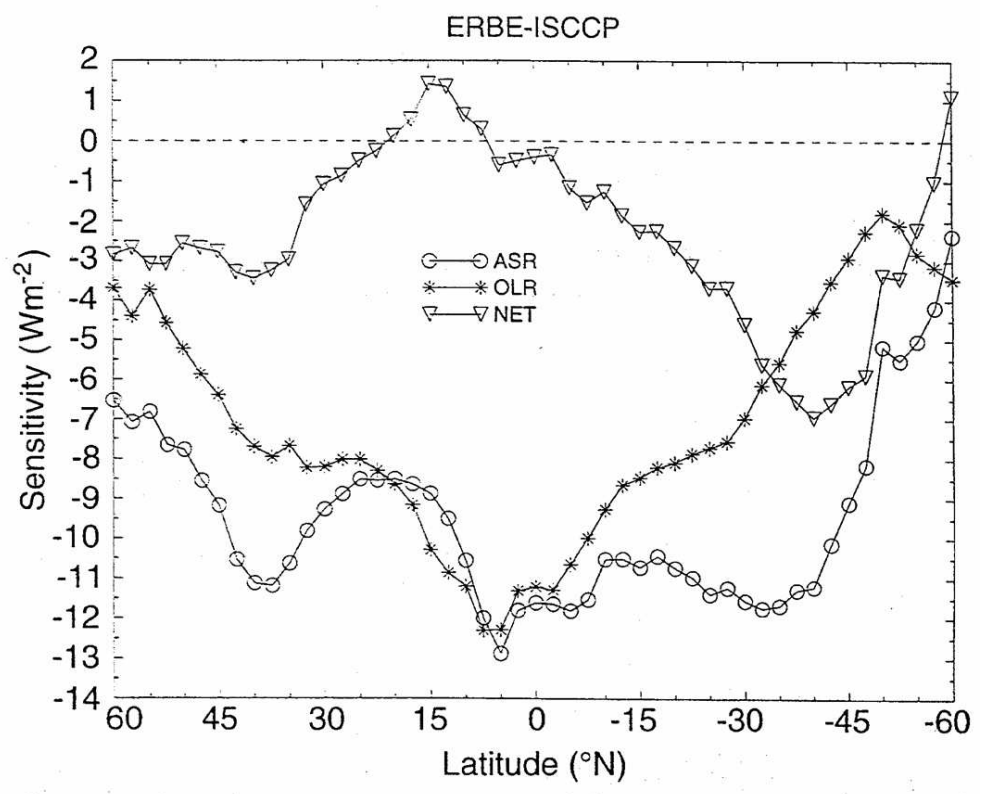

Figure 44: Sensitivity (in $W^{-2}$ per 0.1 increase in fractional cloud cover) of radiation fields (from ERBE data) to cloud cover (from ISCCP data). The curve labelled ASR shows the response of absorbed solar radiation to the presence of cloud; that labelled OLR the response of outgoing longwave radiation. From Ringer and Shine (1997).

place. Due to the energy associated with the surface tension of a droplet it is energetically unfavourable for a small droplet to grow and the water vapour requires a suitable surface, called a condensation nucleus, on which to condense. If the condensation nucleus is not a water surface then heterogeneous nucleation is said to take place; if it is then homogeneous nucleation occurs. In the free atmosphere, however, heterogeneous nucleation is the only important process because homogeneous nucleation requires prohibitively high relative humidities. (For a comprehensive discussion of cloud formation see the classic text by Ludlam, 1980).

Particles which act as condensation nuclei include sea salt, sulphates, mineral dust and aerosols produced from biomass burning. The concentration and composition of atmospheric aerosol vary geographically with, for example, sulphate aerosol being more abundant in the northern hemisphere as it is generated in industrial regions. A region with a higher concentration of condensation nuclei will produce a larger number of smaller cloud droplets than a remote area with clean air which will produce fewer larger droplets for the same total water content.

Smaller drops are more effective at scattering radiation so that the cloud produced in air with more aerosol has a higher albedo. An example of this can be seen in Figure 45 which shows a satellite image in which emissions of carbonaceous aerosol from ships' funnels have modified the reflectivity of a pre-existing deck of stratocumulus cloud.

\subsection{Atmospheric ionisation and cloud}

It has been proposed, originally by Dickinson (1975) who acknowledged that his idea was entirely speculative at the time, that variations in cosmic rays could provide a mechanism whereby solar activity would produce a direct impact on cloud cover by modulating atmospheric ionization, resulting in the electrification of aerosol and increasing its effectiveness to act as condensation nuclei.

Living Reviews in Solar Physics

http://www. livingreviews . org/lrsp-2007-2 


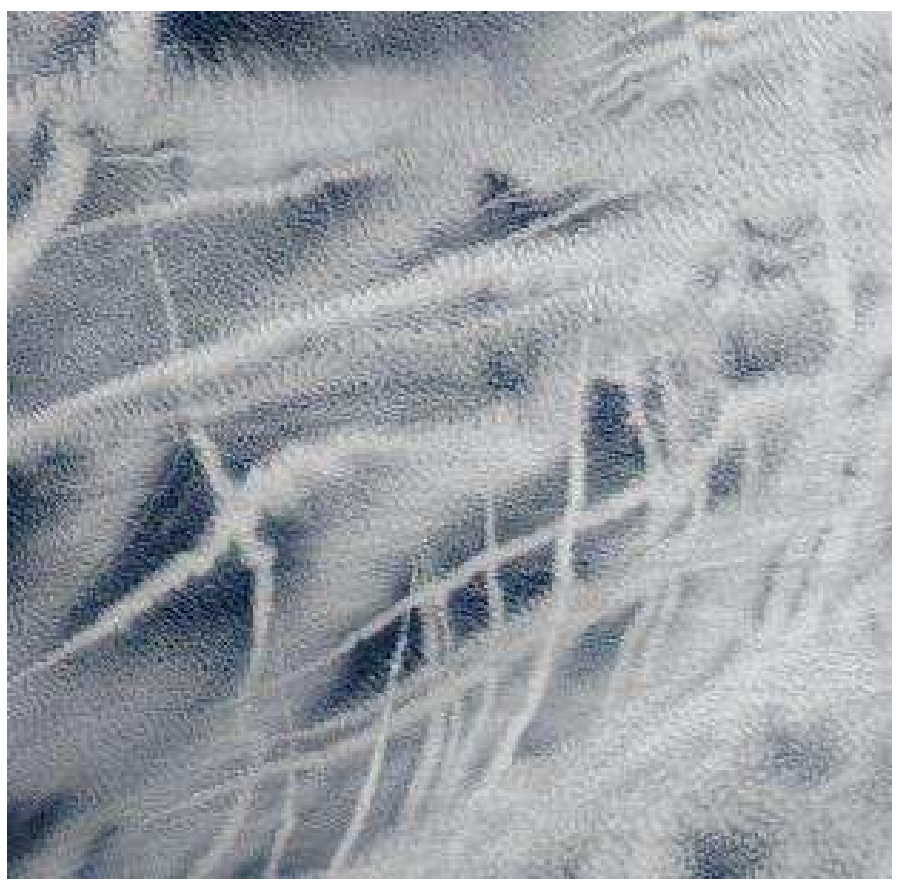

Figure 45: Ship tracks observed off the west coast of France by the MODIS instrument on the Aqua satellite on 27 January 2003. From http: //visibleearth. nasa. gov/.

The processes involved are complex but if they do take place then there is scope for considerable amendment to the value for solar radiative forcing of climate based on incident irradiance alone. Other processes, whereby changes in the Earth's electric field might modify cloud cover have also been proposed (see e.g. Tinsley, 2000) but these are not discussed here.

Ionisation of molecules in the lower atmosphere is brought about by cosmic rays and by naturally-occurring radioactivity. The latter consists of airborne alpha-particle emitters (such as radon gas) and direct gamma radiation from the soil. Cosmic radiation consists of extremely high energy $(>\mathrm{GeV}$ ) particles, mostly protons and helium nuclei. Both sources cause an electron to separate from a molecule of nitrogen or oxygen; the electron then being captured by a neutral molecule on a very short timescale. Thus equal numbers of positive and negative ions are produced. Other processes can introduce a net charge into the atmosphere; these include combustion, rainfall and breaking ocean waves.

Cosmic rays are responsible for approximately $20 \%$ of the ionisation over land surfaces and provide the principle source of ionisation over the oceans. The ionisation rate increases with altitude reaching a peak near $15 \mathrm{~km}$ (see Figure 46a). The geographical distribution of cosmic ray ionisation is strongly modulated by geomagnetism with the rays tending to follow the magnetic field lines down to the magnetic poles. Thus only very high energy cosmic radiation reaches the Earth's surface in low latitudes while much lower energy rays penetrate at high latitudes.

Solar activity modulates the heliospheric magnetic field which acts as a shield to cosmic rays. Thus, during periods of higher solar activity fewer cosmic rays reach the Earth, although the modulation primarily affects lower energy cosmic radiation. At the Earth's surface cosmic rays are monitored by neutron monitors which detect the disintegrated particles (e.g. pions, muons) produced when cosmic radiation impacts atmospheric particles. Figure $46 \mathrm{~b}$ presents a time series of the neutron count rate at two surface stations. The lower latitude station clearly shows lower 
counts and a weaker solar cycle modulation.

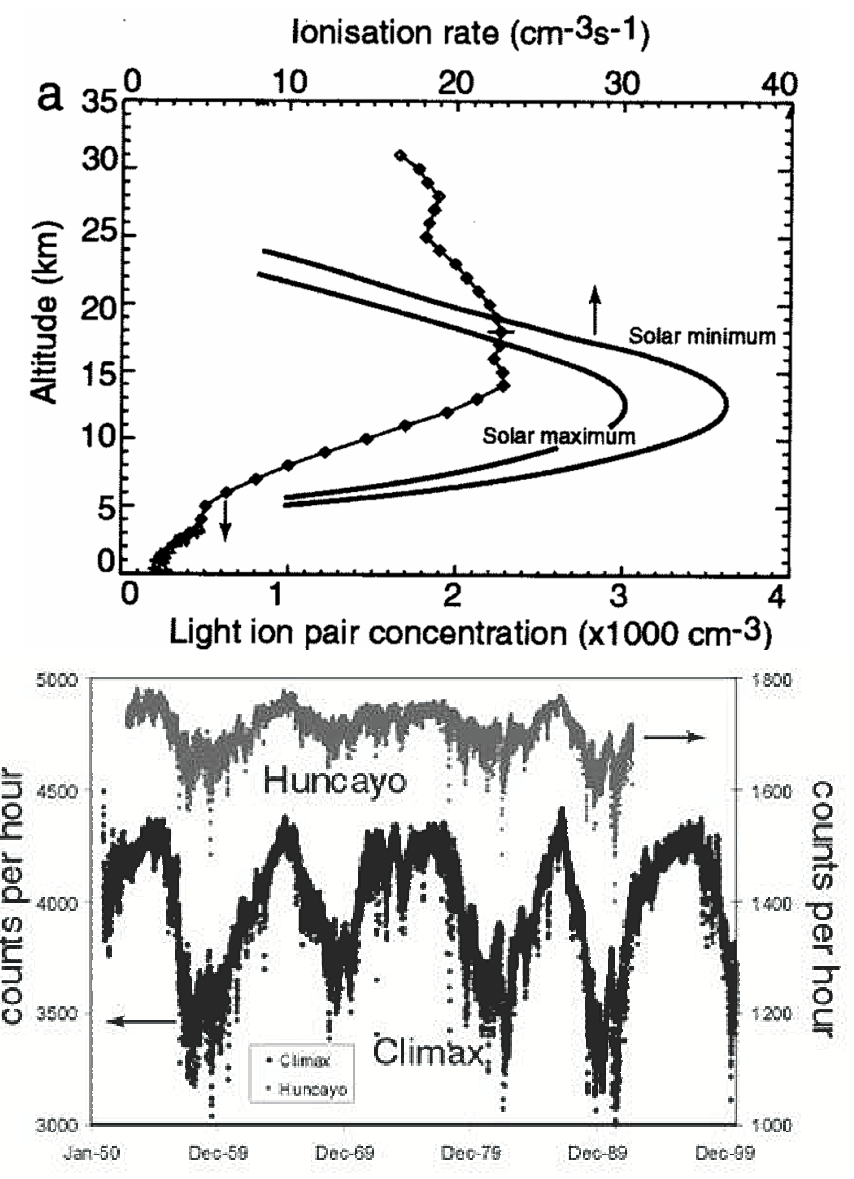

Figure 46: (a) Variation of ionisation rate, and typical ion concentration, with height. (b) Variation with time of the neutron count rate at two surface stations: Climax, latitude $39.4^{\circ} \mathrm{N}$ and Huancayo, latitude 12.0 S. (Harrison and Carslaw, 2003)

The mechanism proposed by Dickinson (1975), and adopted by Marsh and Svensmark (2000) to account for the correlation of tropical marine low cloud cover and cosmic radiation in Figure 10, requires that modulation by solar variability of cosmic rays causes a response in the concentration of cloud condensation nuclei. Several consecutive processes (summarised in Figure 47) need to take place in order for this to come about. Firstly air ions are produced by the action of cosmic rays; this is not controversial, as discussed above, but it should be noted that these ions cannot act directly as cloud condensation nuclei as their small size would require the surrounding air to have a very high level of supersaturation which does not occur in the atmosphere.

The air ions produced by the cosmic rays may act as sites for the nucleation of new ultrafine aerosol (or condensation nuclei, CN). The mechanism then hinges on the extent to which these $\mathrm{CN}$ may grow into particles large enough $(>80 \mathrm{~nm})$ to become cloud condensation nuclei $(\mathrm{CCN})$ and whether this process is enhanced by the particles being charged. Growth may occur through condensation of water vapour or other soluble gases or through coagulation among neutral and charged particles. Some observational evidence (Yu and Turco, 2000) suggests that charged molecular clusters grow faster than neutral clusters and chemical box models (Yu and Turco, 2001) have

Living Reviews in Solar Physics

http://www. livingreviews.org/lrsp-2007-2 


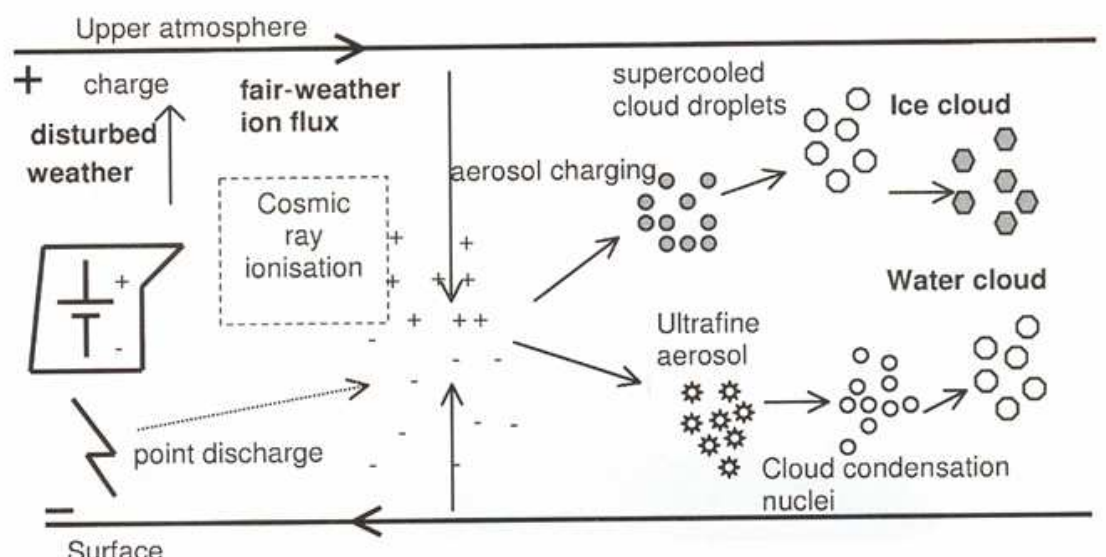

Figure 47: Stages in process whereby atmospheric ionisation results in the formation of cloud droplets/crystals. Harrison and Carslaw (2003)

been able to simulate this effect. However, to reach CCN size would take several days and whether the growth can be maintained depends on the supply of vapour and competitive sources of new aerosol and CCN all of which vary with location, altitude and time of year. Yu (2002) suggests that conditions in the lower troposphere may be more favourable than at higher altitudes but Arnold (2006) concludes that such processes are most likely to occur in the upper troposphere, providing there is a sufficient supply of $\mathrm{SO}_{2}$.

Even if by such a mechanism it proves feasible to produce a measurable effect on cloud cover or properties, the magnitude, and even the sign, of the impact on radiative forcing remains uncertain as it will depend on the cloud location, altitude and physical properties. 


\section{Conclusions}

Radiation from the Sun ultimately provides the only energy source for the Earth's atmosphere and changes in solar activity clearly have the potential to affect climate. There is statistical evidence for solar influence on various meteorological parameters on all timescales, although extracting the signal from the noise in a naturally highly variable system remains a key problem. Changes in total solar irradiance undoubtedly impact the Earth's energy balance but uncertainties in the historical record of TSI mean that the magnitude of even this direct influence is not well known. Variations in solar UV radiation impact the thermal structure and composition of the middle atmosphere but details of the responses in both temperature and ozone concentrations are not well established. Various theories are now being developed for coupling mechanisms whereby direct solar impacts on the middle atmosphere might influence the troposphere but the influences are complex and non-linear and many questions remain concerning the detailed mechanisms which determine to what extent, where and when the solar influence is felt. Variations in cosmic radiation, modulated by solar activity, are manifest in changes in atmospheric ionisation but it is not yet clear whether these have the potential to significantly affect the atmosphere in a way that will impact climate.

Further advances in this field require work on a number of fronts. One important issue is to establish the magnitude of any secular trends in total solar irradiance (TSI). This may be achieved by careful analysis and understanding of the satellite instruments involved in collecting data over the past two-and-a-half solar cycles, and must be continued through analysis of data from current and new satellites. For longer periods it requires a more fundamental understanding of how solar magnetic activity relates to TSI. This would not only facilitate more reliable centennialscale reconstructions of TSI, from e.g. sunspot records, but also advance understanding of how cosmogenic isotope records may be interpreted as historical TSI.

With regard to the climate, further data-mining and analysis are required to firmly establish the magnitude, geographical distribution and seasonality of its response to various forms of solar activity. Understanding the mechanisms involved in the response then becomes the overriding objective. Current ideas suggest three main avenues where further research is needed. Firstly, the means whereby solar radiative heating of the upper and middle atmosphere may influence the lower atmosphere through dynamical coupling needs to be better understood. Secondly, it needs to be established whether or not variations in direct solar heating of the tropical oceans can be of sufficient magnitude to produce apparently observed effects. Thirdly, more work is needed on the microphysical processes involved in ion-induced nucleation, and, probably more importantly, the growth rates of the condensation nuclei produced.

Perhaps when these questions are answered we will be confident that we really understand how changes in the Sun affect the climate on Earth.

Living Reviews in Solar Physics

http://www. livingreviews . org/lrsp-2007-2 


\section{References}

Andrews, D.G., 2000, An Introduction to Atmospheric Physics, Cambridge University Press, Cambridge, U.K.; New York, U.S.A. 24, 28

Arnold, F., 2006, "Atmospheric Aerosol and Cloud Condensation Nuclei Formation: A Possible Influence of Cosmic Rays?", Space Sci. Rev., 125, 169-186.

ADS: http://adsabs.harvard.edu/abs/2006SSRv..125..169A 7.3

Austin, J. et al., 2007, "Coupled chemistry climate simulations of the solar cycle in stratospheric ozone and temperature", J. Geophys. Res., submitted 6.2

Boberg, F., Lundstedt, H., 2002, "Solar Wind Variations Related to Fluctuations of the North Atlantic Oscillation", Geophys. Res. Lett., 29, 1718. Related online version (cited on 06 September 2007):

http://sunspot.lund.irf.se/.

ADS: http://adsabs.harvard.edu/abs/2002GeoRL..29o..13B 2.2

Bond, G., Kromer, B., Beer, J., Muscheler, R., Evans, M.N., Showers, W., Hoffmann, S., LottiBond, R., Hajdas, I., Bonani, G., 2001, "Persistent Solar Influence on North Atlantic Climate During the Holocene", Science, 294, 2130-2136.

ADS: http://adsabs.harvard.edu/abs/2001Sci...294.2130B 2.1, 4

Brasseur, G., 1993, "The response of the middle atmosphere to long-term and short-term solar variability: A two-dimensional model", J. Geophys. Res., 98, 23 079-23090.

ADS: http://adsabs.harvard.edu/abs/1993JGR...9823079B 37

Burroughs, W.J., 1992, Weather Cycles: Real or Imaginary, Cambridge University Press, Cambridge, U.K.; New York, U.S.A. 2.1, 20

Crowley, T.J., 2000, "Causes of Climate Change Over the Past 1000 Years", Science, 289, 270277.

ADS: http://adsabs.harvard.edu/abs/2000Sci...289..270C 32

Dickinson, R.E., 1975, "Solar Variability and the Lower Atmosphere", Bull. Am. Meteorol. Soc., 56, 1240-1248.

ADS: http://adsabs.harvard.edu/abs/1975BAMS ...56.1240D 7.3, 7.3

Eddy, J.A., 1976, “The Maunder Minimum”, Science, 192, 1189-12025

Egorova, T., Rozanov, E., Manzini, E., Haberreiter, M., Schmutz, W., Zubov, V., Peter, T., 2004, "Chemical and dynamical response to the 11-year variability of the solar irradiance simulated with a chemistry climate model", Geophys. Res. Lett., 31, L06 119.

ADS: http://adsabs.harvard.edu/abs/2004GeoRL. .3106119E 37

Foster, S., 2004, Reconstruction of solar irradiance variations, for use in studies of global climate change: application of recent SoHO observations with historic data from the Greenwich Observatory, Ph.D. Thesis, University of Southampton, Southampton, U.K.

ADS: http://adsabs.harvard.edu/abs/2004PhDT.......5F 22

Friis-Christensen, E., Lassen, K., 1991, "Length of the Solar Cycle: An Indicator of Solar Activity Closely Associated with Climate", Science, 254, 698-700.

ADS: http://adsabs.harvard.edu/abs/1991Sci..254..698F 2.2, 6 
Gillett, N.P., Thompson, D.W.J., 2003, "Simulation of Recent Southern Hemisphere Climate Change", Science, 302, 273-275.

ADS: http://adsabs.harvard.edu/abs/2003Sci...302..273G 6.5

Gleisner, H., Thejll, P., 2003, "Patterns of tropospheric response to solar variability", Geophys. Res. Lett., 30, 1711.

ADS: http://adsabs.harvard.edu/abs/2003GeoRL. .30m. .44G 17

Gray, L.J., 2003, "The influence of the equatorial upper stratosphere on sudden stratospheric warmings", Geophys. Res. Lett., 30.

ADS: http://adsabs.harvard.edu/abs/2003GeoRL..30d..15G 6.3

Gray, L.J., Phipps, S.J., Dunkerton, T.J., Baldwin, M.P., Drysdale, E.F., Allen, M.R., 2001, "A data study of the influence of the equatorial upper stratosphere on northern-hemisphere stratospheric sudden warmings", Quart. J. R. Meteorol. Soc., 127, 1985-2003.

ADS: http://adsabs.harvard.edu/abs/2001QJRMS.127.1985G 6.3

Gray, L.J., Crooks, S.A., Pascoe, C., Sparrow, S., Palmer, M., 2004, "Solar and QBO Influences on the Timing of Stratospheric Sudden Warmings", J. Atmos. Sci., 61, 2777-2796.

ADS: http://adsabs.harvard.edu/abs/2004JAtS. .61.2777G 39

Gray, L.J., Haigh, J.D., Harrison, R.G., 2005, The Influence of Solar Changes on the Earth's Climate, Hadley Centre technical note 62, Met Office, Exeter, U.K. URL (cited on 03 September 2007):

http://www.metoffice.gov.uk/research/hadleycentre/pubs/HCTN/index.html 10

Haigh, J.D., 1994, "The role of stratospheric ozone in modulating the solar radiative forcing of climate", Nature, 370, 544-546.

ADS: http://adsabs.harvard.edu/abs/1994Natur.370 . 544H 5.5, 3, 31, 37, 40

Haigh, J.D., 1996, "The Impact of Solar Variability on Climate", Science, 272, 981-984.

ADS: http://adsabs.harvard.edu/abs/1996Sci...272..981H 6.4, 40

Haigh, J.D., 1999, "A GCM study of climate change in response to the 11-year solar cycle", Quart. J. R. Meteorol. Soc., 125, 871-892.

ADS: http://adsabs.harvard.edu/abs/1999QJRMS.125..871H 40

Haigh, J.D., 2003, "The effects of solar variability on the Earth's climate", Philos. Trans. R. Soc. London, Ser. A, 361, 95-111.

ADS: http://adsabs.harvard.edu/abs/2003RSPTA.361_..95H 13

Haigh, J.D., Blackburn, M., 2006, "Solar Influences on Dynamical Coupling Between the Stratosphere and Troposphere", Space Sci. Rev., 125, 331-344.

ADS: http://adsabs.harvard.edu/abs/2006SSRv . .125..331H 6.5, 6.5

Haigh, J.D., Roscoe, H.K., 2006, "Solar influences on polar modes of variability", Meteorol. Z., 15, $371-3782.2,2$

Haigh, J.D., Austin, J., Butchart, N., Chanin, M.-L., Crooks, S., Gray, L.J., Halenka, T., Hampson, J., Hood, L.L., Isaksen, I.S.A., Keckhut, P., Labitzke, K., Langematz, U., Matthes, K., Palmer, M., Rognerud, B., Tourpali, K., Zerefos, C., 2004, "Solar variability and climate: selected results from the SOLICE project", SPARC Newsletter, 23, 19-29. URL (cited on 03 September 2007): http://www.atmosp.physics.utoronto.ca/SPARC/News23/23_Haigh.html 14, 36

Living Reviews in Solar Physics

http://www. livingreviews.org/lrsp-2007-2 
Haigh, J.D., Blackburn, M., Day, R., 2005, "The response of tropospheric circulation to perturbations in lower stratospheric temperature", J. Climate, 18, 3672-3685 16, 6.5, 42, 43

Hansen, J.E., Sato, M., Ruedy, R., 1997, "Radiative forcing and climate response", J. Geophys. Res., 102, 6831-6864.

ADS: http://adsabs.harvard.edu/abs/1997JGR...102.6831H 3

Harrison, R.G., Carslaw, K.S., 2003, "Ion-aerosol-cloud processes in the lower atmosphere", Rev. Geophys., 41, 1012.

ADS: http://adsabs.harvard.edu/abs/2003RvGeo..41c...2H 46, 47

Houghton, J.T., 1977, The Physics of Atmospheres, Cambridge University Press, Cambridge, U.K.; New York, U.S.A. 23

Hoyt, D.V., Schatten, K.H., 1998, "Group Sunspot Numbers: A New Solar Activity Reconstruction", Solar Phys., 179, 189-219.

ADS: http://adsabs.harvard.edu/abs/1998SoPh. .181..491H 4.2, 6.1

IPCC, 2001, Climate Change 2001: The Scientific Basis. Contribution of Working Group I to the Third Assessment Report of the Intergovernmental Panel on Climate Change, Cambridge University Press, Cambridge, U.K.; New York, U.S.A. Related online version (cited on 03 September 2007):

http://www.grida.no/climate/ipcc_tar/wg1/index.htm. (Eds.) Houghton, J.T. and Ding, Y. and Griggs, D.J. and Noguer, M. and van der Linden, P.J. and Dai, X. and Maskell, K. and Johnson, C.A. 1, 2

IPCC, 2007, Climate Change 200\%: The Physical Science Basis. Contribution of Working Group I to the Fourth Assessment Report of the Intergovernmental Panel on Climate Change, Cambridge University Press, Cambridge, U.K.; New York, U.S.A. Related online version (cited on 03 September 2007):

http://ipcc-wg1.ucar.edu/wg1/wg1-report.html. (Eds.) Solomon, S. and Qin, D. and Manning, M. and Chen, Y. and Marquis, M. and Averyt, K.B. and Tignor, M. and Miller, H.L. 2.1, 19, 33

Jackman, C.H., DeLand, M.T., Labow, G.J., Fleming, E.L., Lópes-Puertas, M., 2006, "Satellite Measurements of Middle Atmospheric Impacts by Solar Proton Events in Solar Cycle 23", Space Sci. Rev., 125, 381-391.

ADS: http://adsabs.harvard.edu/abs/2006SSRv. .125..381J 5.4

Kiehl, J.T., Trenberth, K.E., 1997, "Earth's Annual Global Mean Energy Budget", Bull. Am. Meteorol. Soc., 78, 197-208. Related online version (cited on 06 September 2007): http://www.cgd.ucar.edu/cas/papers/KiehlTrenbBAMS97.pdf 18

Kodera, K., 1995, "On the origin and nature of the interannual variability of the winter stratospheric circulation in the northern hemisphere", J. Geophys. Res., 100, 14077-14087.

ADS: http://adsabs.harvard.edu/abs/1995JGR...10014077K 6.3

Kodera, K., Kuroda, Y., 2002, "Dynamical response to the solar cycle", J. Geophys. Res., 107, 2224 .

ADS: http://adsabs.harvard.edu/abs/2002JGRD.107x.ACL5K 6.3, 38

Kristjansson, J.E., Kristiansen, J., 2000, "Is there a cosmic ray signal in recent variations in global cloudiness and cloud radiative forcing?", J. Geophys. Res., 105, 11851-11863.

ADS: http://adsabs.harvard.edu/abs/2000JGR...10511851K 2.2 
Kushner, P.J., Polvani, L.M., 2004, "Stratosphere-troposphere coupling in a relatively simple AGCM: The role of eddies", J. Climate, 17, 629-639.

ADS: http://adsabs.harvard.edu/abs/2004JCli...17..629K 6.5

Labitzke, K., van Loon, H., 1992, "On the association between the QBO and the extratropical stratosphere", J. Atmos. Terr. Phys., 54, 1453-1463 15, 6.3

Labitzke, K., van Loon, H., 1995, "Connection between the troposphere and stratosphere on a decadal scale", Tellus A, 47, 275-286.

ADS: http://adsabs.harvard.edu/abs/1995TellA..47..275L 11

Larkin, A., 2000, Investigation into the effects of solar variability on climate using atmospheric models of the troposphere and stratosphere, Ph.D. Thesis, University of London, London, U.K. 27,41

Larkin, A., Haigh, J.D., Djavidnia, S., 2000, "The Effect of Solar UV Irradiance Variations on the Earth's Atmosphere", Space Sci. Rev., 94, 199-214.

ADS: http://adsabs.harvard.edu/abs/2000SSRv...94..199L 3, 41

Laut, P., Gundermann, J., 2000, "Solar cycle lengths and climate: A reference revisited", J. Geophys. Res., 105, $27489-27492$.

ADS: http://adsabs.harvard.edu/abs/2000JGR...10527489L 2.2, 7

Lean, J., 1989, "Contribution Of Ultraviolet Irradiance Variations To Changes In The Sun's Total Irradiance", Science, 244, 197-200.

ADS: http://adsabs.harvard.edu/abs/1989Sci...244..197L 40

Lean, J., 2000, "Evolution of the Sun's spectral irradiance since the Maunder Minimum", Geophys. Res. Lett., 27, 2425-2428.

ADS: http://adsabs.harvard.edu/abs/2000GeoRL. .27.2425L 22, 26

Lee, H., Smith, A.K., 2003, "Simulation of the combined effects of solar cycle, quasi-biennial oscillation and volcanic forcing on stratospheric ozone changes in recent decades", J. Geophys. Res., 108, 1503.

ADS: http://adsabs.harvard.edu/abs/2003JGRD..108.4049L 6.2

Ludlam, F.H., 1980, Clouds and Storms: The Behavior and Effect of Water in the Atmosphere, Pennylvania State University Press, University Park, U.S.A. 7.2

Mann, M.E., Bradley, R.S., Hughes, M.K., 1999, "Northern hemisphere temperatures during the past millennium: Inferences, uncertainties, and limitations", Geophys. Res. Lett., 26, 759-762. ADS: http://adsabs.harvard.edu/abs/1999GeoRL. .26 . .759M 7

Marsh, N.D., Svensmark, H., 2000, "Low Cloud Properties Influenced by Cosmic Rays", Phys. Rev. Lett., 85, 5004-5007.

ADS: http://adsabs.harvard.edu/abs/2000PhRvL. .85.5004M 2.2, 7.3

Marsh, N.D., Svensmark, H., 2003, "Solar Influence on Earth's Climate", Space Sci. Rev., 107, 317-325.

ADS: http://adsabs.harvard.edu/abs/2003SSRv . 107 . .317M 10

Matthes, K., Kodera, K., Haigh, J.D., Shindell, D.T., Shibata, K., Langematz, U., Rozanov, E., Kuroda, Y., 2003, "GRIPS Solar Experiments Intercomparison Project: Initial Results", Pap. Met. Geophys., 54, 71-90 35 
Matthes, K., Langematz, U., Gray, L.J., Kodera, K., Labitzke, K., 2004, "Realistic solar signal in the FUB-CMAM", J. Geophys. Res., 1096.3

Meehl, G.A., Washington, W.M., Wigley, T.M.L., Arblaster, J.M., Dai, A., 2003, "Solar and greenhouse gas forcing and climate response in the twentieth century", J. Climate, 16, 426-444. ADS: http://adsabs.harvard.edu/abs/2003JCli...16..426M 6.1

Myhre, G., Stordal, F., Rognerud, B., Isaksen, I.S.A., 1998, "Radiative forcing due to stratospheric ozone", in Atmospheric Ozone, (Eds.) Bojkov, R.D., Visconti, G., Proceedings of the XVIII Quadrennial Ozone Symposium, L'Aquila, Italy, September 12-21, 1996, pp. 813-816, Edigrafital S.P.A. / Parco Scientifico e Tecnologico d'Abruzzo, Sant'Atto, Italy 3

Petit, J.R., Jouzel, J., Raynaud, D., Barkov, N.I., Barnola, J.M., Basile, I., Bender, M., Chappellaz, J., Davis, M., Delaygue, G., Delmotte, M., Kotlyakov, V.M., Legrand, M., Lipenkov, V.Y., Lorius, C., Pépin, L., Ritz, C., Saltzman, E., Stievenard, M., 1999, "Climate and atmospheric history of the past 420,000 years from the Vostok ice core, Antarctica", Nature, 399, 429-436 4.1

Polvani, L.M., Kushner, P.J., 2002, "Tropospheric response to stratospheric perturbations in a relatively simple general circulation model", Geophys. Res. Lett., 29, 1114.

ADS: http://adsabs.harvard.edu/abs/2002GeoRL..29g. .18P 6.5

Randel, W.J., Wu, F., 2007, "A stratospheric ozone profile data set for 1979-2005: Variability, trends, and comparisons with column ozone data", J. Geophys. Res., 112.

ADS: http://adsabs.harvard.edu/abs/2007JGRD..11206313R 29

Ringer, M.A., Shine, K.P., 1997, "Sensitivity of the Earth's radiation budget to interannual variations in cloud amount", Climate Dyn., 13, 213-222.

ADS: http://adsabs.harvard.edu/abs/1997ClDy...13..213R 44

Schmidt, H., Brasseur, G.P., 2006, "The Response of the Middle Atmosphere to Solar Cycle Forcing in the Hamburg Model of the Neutral and Ionized Atmosphere", Space Sci. Rev., 125, 345-356. ADS: http://adsabs.harvard.edu/abs/2006SSRv..125..345S 6.2

Shindell, D.T., Schmidt, G.A., Mann, M.E., Rind, D., Waple, A., 2001, "Solar Forcing of Regional Climate Change During the Maunder Minimum", Science, 294, 2149-2152.

ADS: http://adsabs.harvard.edu/abs/2001Sci...294.2149S 3

Shindell, D.T., Faluvegi, G., Miller, R.L., Schmidt, G.A., Hansen, J.E., Sun, S., 2006, "Solar and anthropogenic forcing of tropical hydrology", Geophys. Res. Lett., 33.

ADS: http://adsabs.harvard.edu/abs/2006GeoRL. .3324706S 6.4

Stauffer, B., 2000, "Long Term Climate Records from Polar Ice", Space Sci. Rev., 94, 321-336. ADS: http://adsabs.harvard.edu/abs/2000SSRv...94..321S 3

Stott, P.A., Jones, G.S., Mitchell, J.F.B., 2003, "Do Models Underestimate the Solar Contribution to Recent Climate Change?", J. Climate, 16, 4079-4093.

ADS: http://adsabs.harvard.edu/abs/2003JCli...16.4079S 34, 6.1

Strobel, D.F., 1978, "Parameterization of the atmospheric heating rate from 15 to $120 \mathrm{~km}$ due to $\mathrm{O}_{2}$ and $\mathrm{O}_{3}$ absorption of solar radiation", J. Geophys. Res., 83, 6225-6230.

ADS: http://adsabs.harvard.edu/abs/1978JGR...83.6225S 25

Thompson, D.W.J., Baldwin, M.P., Solomon, S., 2005, "Stratosphere-Troposphere Coupling in the Southern Hemisphere", J. Atmos. Sci., 62, 708-715.

ADS: http://adsabs.harvard.edu/abs/2005JAtS..62..708T 6.5 
Tinsley, B.A., 2000, "Influence of the Solar Wind on the Global Electric Circuit and Inferred Effects on Cloud Microphysics, Temperature and Dynamics in the Troposphere", Space Sci. Rev., 94, 231-258.

ADS: http://adsabs.harvard.edu/abs/2000SSRv...94..231T 7.3

Tourpali, K., Schuurmans, C.J.E., van Dorland, R., Steil, B., Brühl, C., 2003, "Stratospheric and tropospheric response to enhanced solar UV radiation: A model study", Geophys. Res. Lett., 30, 1231.

ADS: http://adsabs.harvard.edu/abs/2003GeoRL. .30e..35T 37

van Loon, H., Shea, D.J., 1999, "A probable signal of the 11-year solar cycle in the troposphere of the northern hemisphere", Geophys. Res. Lett., 26, 2893-2896.

ADS: http://adsabs.harvard.edu/abs/1999GeoRL..26.2893V 12

van Loon, H., Meehl, G.A., Shea, D.J., 2007, "Coupled air-sea response to solar forcing in the Pacific region during northern winter", J. Geophys. Res., 112, D02108 9

Vlachogiannis, D., Haigh, J.D., 1998, "The impact of solar proton events on lower stratospheric ozone", in Atmospheric Ozone, (Eds.) Bojkov, R.D., Visconti, G., Proceedings of the XVIII Quadrennial Ozone Symposium, L'Aquila, Italy, September 12-21, 1996, pp. 275-278, Edigrafital S.P.A. / Parco Scientifico e Tecnologico d'Abruzzo, Sant'Atto, Italy 30

Wang, Y.-M., Lean, J.L., Sheeley Jr, N.R., 2005, "Modeling the Sun's Magnetic Field and Irradiance since 1713", Astrophys. J., 625, 522-538.

ADS: http://adsabs.harvard.edu/abs/2005ApJ...625..522W 22

White, W.B., Lean, J.L., Cayan, D.R., Dettinger, M.D., 1997, "Response of global upper ocean temperature to changing solar irradiance", J. Geophys. Res., 102, 3255-3266.

ADS: http://adsabs.harvard.edu/abs/1997JGR...102.3255W 8, 2.3

Willson, R.C., Mordinov, A.V., 2003, "Secular total solar irradiance trend during solar cycles 2123", Geophys. Res. Lett., 30, 1199-1202.

ADS: http://adsabs.harvard.edu/abs/2003GeoRL..30e...3W 4.2

WMO, 2007, Scientific Assessment of Ozone Depletion: 2006, Global Ozone Monitoring Project - Report No. 50, World Meteorological Organization, Geneva, Switzerland. URL (cited on 03 September 2007):

http://www.wmo.int/pages/prog/arep/gaw/ozone_2006/ozone_asst_report.html 37

Wuebbles, D.J., Wei, C.-F., Patten, K.O., 1998, "Effects on stratospheric ozone and temperature during the Maunder Minimum", Geophys. Res. Lett., 25, 523-526.

ADS: http://adsabs.harvard.edu/abs/1998GeoRL . 25 . 523W 3

Yu, F., 2002, "Altitude variations of cosmic ray induced production of aerosols: Implications for global cloudiness and climate", J. Geophys. Res., 107.

ADS: http://adsabs.harvard.edu/abs/2002JGRA.107g.SIA8Y 7.3

Yu, F., Turco, R.P., 2000, "Ultrafine aerosol formation via ion-mediated nucleation", Geophys. Res. Lett., 27, 883-886.

ADS: http://adsabs.harvard.edu/abs/2000GeoRL. .27. .883Y 7.3

Yu, F., Turco, R.P., 2001, "From molecular clusters to nanoparticles: Role of ambient ionization in tropospheric aerosol formation", J. Geophys. Res., 106, 4797-4814.

ADS: http://adsabs.harvard.edu/abs/2001JGR...106.4797Y 7.3

Living Reviews in Solar Physics

http://www. livingreviews.org/lrsp-2007-2 